\title{
Resolvent and propagation estimates for Klein-Gordon equations with non-positive energy
}

\author{
Vladimir Georgescu, Christian Gérard, and Dietrich Häfner
}

Abstract. We study in this paper an abstract class of Klein-Gordon equations

$$
\partial_{t}^{2} \phi(t)-2 \mathrm{i} k \partial_{t} \phi(t)+h \phi(t)=0,
$$

where $\phi: \mathbb{R} \rightarrow \mathcal{H}, \mathcal{H}$ is a (complex) Hilbert space, and $h, k$ are self-adjoint, resp. symmetric operators on $\mathcal{H}$.

We consider their generators $H$ (resp. $K$ ) in the two natural spaces of Cauchy data, the energy (resp. charge) spaces. We do not assume that the dynamics generated by $H$ or $K$ has any positive conserved quantity, in particular these operators may have complex spectrum. Assuming conditions on $h$ and $k$ which allow to use the theory of selfadjoint operators on Krein spaces, we prove weighted estimates on the boundary values of the resolvents of $H$, $K$ on the real axis. From these resolvent estimates we obtain corresponding propagation estimates on the behavior of the dynamics for large times.

Examples include wave or Klein-Gordon equations on asymptotically euclidean or asymptotically hyperbolic manifolds, minimally coupled with an external electro-magnetic field decaying at infinity.

Mathematics Subject Classification (2010). 35L05, 35P25, 81U, 81Q05, 81Q12.

Keywords. Klein-Gordon equations, Krein spaces, resolvent estimates, propagation estimates. 


\section{Contents}

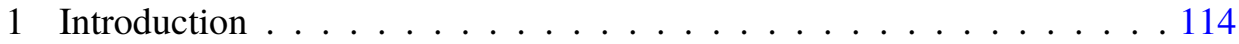

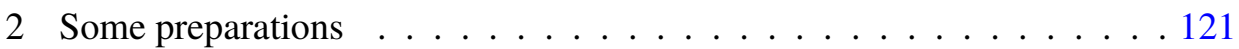

3 Operators on Krein spaces . . . . . . . . . . . . . . . . 125

4 Abstract Klein-Gordon equations . . . . . . . . . . . . . . . . . 134

5 Klein-Gordon operators in energy spaces . . . . . . . . . . . . . 138

6 Klein-Gordon operators in charge spaces . . . . . . . . . . . . . . . . 144

7 Definitizable Klein-Gordon operators on energy spaces . . . . . . . . 149

8 Mourre estimate for Klein-Gordon operators on energy spaces . . . . 157

9 Limiting absorption principle . . . . . . . . . . . . . . 162

10 Existence of the dynamics . . . . . . . . . . . . . . 166

11 Propagation estimates . . . . . . . . . . . . . . . . . . 167

12 Examples . . . . . . . . . . . . . . . . . 170

Appendix A . . . . . . . . . . . . . . . . . . 181

Appendix B . . . . . . . . . . . . . . . . . . . . 187

References . . . . . . . . . . . . . . . . . . . . 190

\section{Introduction}

This paper is devoted to the proof of resolvent and propagation estimates for the generators of a class of abstract Klein-Gordon equations

$$
\partial_{t}^{2} \phi(t)-2 \mathrm{i} k \partial_{t} \phi(t)+h \phi(t)=0,
$$

where $\phi: \mathbb{R} \rightarrow \mathcal{H}, \mathcal{H}$ is a (complex) Hilbert space, and $h, k$ are self-adjoint, resp. symmetric operators on $\mathcal{H}$.

There are many natural examples of such abstract class of equations: one class is obtained by considering Klein-Gordon equations

$$
-\nabla^{a} \nabla_{a} \phi+m^{2} \phi=0
$$

on a Lorentzian manifold having a global Killing vector field, corresponding in (1.1) to $\partial_{t}$. A related class is obtained by perturbing a static Klein-Gordon equation

$$
\partial_{t}^{2} \phi-\nabla^{j} \nabla_{j} \phi+m^{2} \phi=0
$$


on $\mathbb{R}_{t} \times N$ ( $N$ is a Riemannian manifold) by minimal coupling this equation to an external electro-magnetic field $A_{a}$ independent on $t$. We obtain then the equation

$$
\left(\partial_{t}-\mathrm{i} v(x)\right)^{2} \phi-\left(\nabla^{j}-\mathrm{i} A^{j}(x)\right)\left(\nabla_{j}-\mathrm{i} A_{j}(x)\right) \phi+m^{2} \phi=0,
$$

which can be put in the form (1.1).

An example to keep in mind is the Klein-Gordon equation on Minkowski space minimally coupled with an external electric field

$$
\left(\partial_{t}-\mathrm{i} v(x)\right)^{2} \phi(t, x)-\Delta_{x} \phi(t, x)+m^{2} \phi(t, x)=0,
$$

for which $\mathcal{H}=L^{2}\left(\mathbb{R}^{d}, d x\right), h=-\Delta_{x}+m^{2}-v^{2}(x), k=v(x)$ is a (real) electric potential and $m \geq 0$ is the mass of the Klein-Gordon field. We will use this example to describe the results and methods of the present work.

1.1. Description of the main results. The equation (1.2) has two natural conserved quantities, the charge

$$
\int_{\mathbb{R}^{d}}\left(\mathrm{i} \partial_{t} \bar{\phi}(t, x) \phi(t, x)-\mathrm{i} \bar{\phi}(t, x) \partial_{t} \phi(t, x)-2 v(x)|\phi(t, x)|^{2}\right) d x,
$$

and the energy

$$
\int_{\mathbb{R}^{d}}\left(\left|\partial_{t} \phi(t, x)\right|^{2}+\left|\nabla_{x} \phi(t, x)\right|^{2}+\left(m^{2}-v^{2}(x)\right)|\phi(t, x)|^{2}\right) d x,
$$

both related to the symplectic nature of (1.2). In order to associate a generator to (1.2), one has to consider a Cauchy problem. There are two natural ways to define Cauchy data at time $t$. On can set

$$
f(t)=\left(\begin{array}{c}
\phi(t) \\
\mathrm{i}^{-1} \partial_{t} \phi(t)-v \phi(t)
\end{array}\right),
$$

so that

$$
f(t)=\mathrm{e}^{\mathrm{i} t K} f(0), \quad K=\left(\begin{array}{cc}
v & \mathbb{1} \\
-\Delta_{x}+m^{2} & v
\end{array}\right) .
$$

This choice is natural when one emphasizes the conservation of the charge, which takes the simple form

$$
q(f, f)=\int_{\mathbb{R}^{d}} \bar{f}_{1}(x) f_{0}(x)+\bar{f}_{0}(x) f_{1}(x) d x, \quad f=\left(\begin{array}{l}
f_{0} \\
f_{1}
\end{array}\right) .
$$


Another choice, more common in the PDE literature is

$$
f(t)=\left(\begin{array}{c}
\phi(t) \\
\mathrm{i}^{-1} \partial_{t} \phi(t)
\end{array}\right)
$$

so that

$$
f(t)=\mathrm{e}^{\mathrm{i} t H} f(0), \quad H=\left(\begin{array}{cc}
0 & \mathbb{1} \\
-\Delta_{x}+m^{2}-v^{2} & 2 v
\end{array}\right) .
$$

With this choice the energy takes the simple form

$$
E(f, f)=\int_{\mathbb{R}^{d}}\left|f_{1}\right|^{2}(x)+\left|\nabla f_{0}\right|^{2}(x)+\left(m^{2}-v^{2}(x)\right)\left|f_{0}\right|^{2}(x) d x, \quad f=\left(\begin{array}{l}
f_{0} \\
f_{1}
\end{array}\right) .
$$

Note that the two operators $K$ and $H$ are obviously related by similarity, see e.g. Subsection 4.4.

The main problem one faces when studying Klein-Gordon equations (1.2) is the lack of a positive conserved quantity. For example $q$ is clearly never positive definite, while $E$ is not positive definite if the electric potential $v$ becomes too large, so that $-\Delta_{x}+m^{2}-v^{2}(x)$ acquires some negative spectrum. In other words it is generally not possible to equip the space of Cauchy data with a Hilbert space structure such that $K$ or $H$ are self-adjoint.

There are two manifestations of this problem with some physical significance. The first one, discovered long ago by physicists [30], is the fact that if $v$ is too large, $K$ and $H$ acquire complex eigenvalues, appearing in complex conjugate pairs. This phenomenon is sometimes called the Klein paradox. It implies the existence of exponentially growing solutions, and causes difficulties with the quantization of (1.2).

The second manifestation is known as superradiance. It appears for example for the Klein-Gordon equation (1.2) in 1 dimension, when the electric potential $v$ has two limits $v_{ \pm}$at $\pm \infty$ with $\left|v_{+}-v_{-}\right|>m$, see [2] for a mathematical analysis. It also appears in more complicated models, like the Klein-Gordon equation on the Kerr space-time, which can be reduced to the abstract form (1.1) after some separation of variables.

Superradiance appears when there exist infinite dimensional subspaces, asymptotic invariant under $H$, on which the energy is positive (resp. negative). If this happens a wave coming from $+\infty$ may, after scattering by the potential, return to $+\infty$ with more energy than it initially had. 
Another more mathematical issue with (1.2) is that there are many possible topologies to put on the space of Cauchy data. If we use (1.3) it is natural to require that the charge should be bounded for the chosen topology. This of course does not fix the topology, but by considering the simple case $v(x) \equiv 0$ it is easy to see (see Subsection 4.5) that the natural space of Cauchy data is the charge space

$$
\mathcal{F}=H^{\frac{1}{2}}\left(\mathbb{R}^{d}\right) \oplus H^{-\frac{1}{2}}\left(\mathbb{R}^{d}\right),
$$

where $H^{s}\left(\mathbb{R}^{d}\right)$ denotes the usual Sobolev space of order $s$.

If we use (1.4), then we should require that the energy be bounded, which leads to the essentially unique choice of the energy space

$$
\mathcal{E}=H^{1}\left(\mathbb{R}^{d}\right) \oplus L^{2}\left(\mathbb{R}^{d}\right)
$$

Note that if $m=0$ the homogeneous energy space

$$
\dot{\mathcal{E}}=\left(-\Delta_{x}-v^{2}\right)^{-\frac{1}{2}} L^{2}\left(\mathbb{R}^{d}\right) \oplus L^{2}\left(\mathbb{R}^{d}\right)
$$

(see Subsection 2.1 for this notation) could also be considered, and will actually play an important role in our work.

Let us now illustrate the results of our paper on the example (1.2), assuming for simplicity that $v \in C_{0}^{\infty}\left(\mathbb{R}^{d}\right)$. Using general results on self-adjoint operators on Krein spaces, one can first show that

$$
\begin{aligned}
\sigma(H) & =\sigma(K), \\
\sigma_{\mathrm{ess}}(H) & \left.\left.=\sigma_{\mathrm{ess}}(K)=\right]-\infty,-m\right] \cup[m,+\infty[, \\
\sigma(H) \backslash \mathbb{R} & =\sigma(K) \backslash \mathbb{R}=\cup_{1 \leq j \leq n}\left\{\lambda_{j}, \overline{\lambda_{j}}\right\},
\end{aligned}
$$

where $\lambda_{j}, \overline{\lambda_{j}}$ are eigenvalues of finite Riesz index.

The main result of this work are weighted resolvent estimates, valid near the essential spectrum of $K, H$,

$$
\sup _{\operatorname{Re} z \in I, 0<\operatorname{Im} z \mid \leq \delta}\left\|\langle x\rangle_{\text {diag }}^{-\delta}(H-z)^{-1}\langle x\rangle_{\text {diag }}^{-\delta}\right\|_{B(\varepsilon)}<\infty, \quad \text { for all } \frac{1}{2}<\delta,
$$

where the brackets $\langle x\rangle_{\text {diag }}$ denote the diagonal operator on the space of Cauchy data $H^{1}\left(\mathbb{R}^{d}\right) \oplus L^{2}\left(\mathbb{R}^{d}\right)$ with entries $\langle x\rangle$ (see Subsection 2.1$), I \subset \mathbb{R}$ is a compact interval disjoint from $\pm m$, containing no real eigenvalues of $H$, nor so called critical points of $H$ (see Section 3 for the definition of critical points). 
Similar results hold for $K$, replacing $\mathcal{E}$ by $\mathcal{F}$. By the usual argument based on Fourier transformation, we deduce from (1.5) propagation estimates on the $C_{0}$-groups $\mathrm{e}^{\mathrm{i} t H}$ and $\mathrm{e}^{\mathrm{i} t K}$

$$
\int_{\mathbb{R}}\left\|\langle x\rangle_{\text {diag }}^{-\delta} \mathrm{e}^{\mathrm{i} t H} \chi(H)\langle x\rangle^{-\delta} f\right\|_{\mathcal{E}}^{2} d t \leq C\|f\|_{\mathcal{E}}^{2},
$$

and

$$
\int_{\mathbb{R}}\left\|\langle x\rangle_{\operatorname{diag}}^{-\delta} \mathrm{e}^{\mathrm{i} t K} \chi(K)\langle x\rangle^{-\delta} f\right\|_{\mathcal{F}}^{2} d t \leq C\|f\|_{\mathcal{F}}^{2},
$$

where $\chi \in C_{0}^{\infty}(\mathbb{R})$ is a cutoff function supported away from real eigenvalues and critical points of $H$ and $K$.

From (1.6) it is easy to construct the short-range scattering theory for the dynamics $\mathrm{e}^{\mathrm{i} t H}, \mathrm{e}^{\mathrm{i} t K}$. With a little more effort, the long-range scattering theory can also be constructed. In this way the results of [11], dealing with the scattering theory of massive Klein-Gordon equations in energy spaces, can certainly be extended to the massless case (ie to wave equations), both in the energy and charge spaces.

1.2. Methods. In the usual Hilbert space setting, where $H$ is self-adjoint for some Hilbert space scalar product, the most powerful way to prove estimates (1.5) and (1.6) relies on the Mourre method, i.e. on the construction of another selfadjoint operator $A$ such that

$$
\mathbb{1}_{I}(H)[H, \mathrm{i} A] \mathbb{1}_{I}(H) \geq c \mathbb{1}_{I}(H)+R,
$$

where $R$ is compact and $c>0$. This method can be directly applied to (1.2) if the energy $E(f, f)$ is positive definite, so that it defines a compatible scalar product on $\mathcal{E}$. Numerous papers rely on this observation, see among many others the papers [6, 25, 27, 29, 34], and for more recent results based on the Mourre method $[13,14]$.

If the energy is not positive, one can consider the energy space $\mathcal{E}$ equipped with $E$ as a Krein space, i.e. a Hilbertizable vector space equipped with a bounded, nondegenerate hermitian form. Orthogonal and adjoints on a Krein space are defined w.r.t. the Krein scalar product, and conservation of energy is formally equivalent to the fact that the generator $H$ is self-adjoint in the Krein sense.

There exists a class of self-adjoint operators on Krein spaces, the so-called definitizable operators, (see Subsection 3.2) which admit a continuous and Borel functional calculus quite similar to the one of usual self-adjoint operators. A finite set of their real spectrum, called the critical points, plays the role of spectral singularities for the functional calculus. Spectral projections on intervals whose 
endpoints are not critical points can be defined, and they have the important property that if they do not contain critical points, then the Krein scalar product is definite (positive or negative) on their range.

In [9], we exploited these properties of definitizable operators on Krein spaces to extend the Mourre method to this setting, obtaining weighted resolvent estimates in an abstract setting.

In this work we apply the general results of [9] to one of the main examples of self-adjoint operators on Krein spaces, namely the generators of one of the $C_{0}$-groups associated to the abstract Klein-Gordon equation (1.1).

Note that several papers were devoted to Klein-Gordon or wave equations from the Krein space point of view, see e.g. [18, 23, 24]. However resolvent estimates near the real spectrum were never considered in the above papers.

We obtain resolvent and propagation estimates which are generalizations of equations (1.5) and (1.6). Examples of our abstract framework are minimally coupled Klein-Gordon or wave equations on scattering or asymptotically hyperbolic manifolds.

Note that the typical assumption of the electric potential $v$ is that it should decay to 0 at $\infty$. This assumption is necessary to ensure that $H$ is definitizable. Therefore the models considered in this paper, while possibly exhibiting the Klein paradox, do not give rise to superradiance. In a subsequent paper [10] we will prove similar results for a model exhibiting superradiance, namely the KleinGordon equation on Kerr-de Sitter space-times. Using the results of this paper, it is possible to prove resolvent estimates and to study scattering theory also for such superradiant Klein-Gordon equations.

1.3. Plan of the paper. Section 2 contains some preparatory material, the most important dealing with quadratic operator pencils.

In Section 3 we recall the theory of definitizable operators on Krein spaces. In particular we devote some effort to present a self-contained exposition of their functional calculus, which is a rather delicate but interesting topic. Among previous contributions to this question, we mention the works of Langer [22] and Jonas [19].

In [9] we constructed the natural version of the continuous functional calculus for a definitizable operator $H$, associated to an algebra of continuous functions having asymptotic expansions of a specific order at each critical point of $H$. Although we will not need its full generality in the rest of the paper, we found it worthwhile to develop the corresponding Borel functional calculus. An interesting feature of this calculus is that the natural algebra is not an algebra of functions 
on $\mathbb{R}$ anymore, but has to be augmented by adding a copy of $\mathbb{C}$ at each critical point.

In Section 4 we discuss in some detail the various setups for abstract KleinGordon equations and the possible choices of topologies on the space of initial data.

Sections 5 and 6 are devoted to basic facts on the generators of Klein-Gordon equations in energy and charge spaces respectively. In particular quadratic pencils play an important role here.

Related results were obtained before by Langer, Najman and Tretter in [23] and [24] for the energy resp. charge Klein-Gordon operators. We will comment on the relationship between our framework and the one in $[23,24]$ in Remarks 5.5 and 6.6.

In Section 7 we introduce a class of definitizable Klein-Gordon operators. We also construct an approximate diagonalization of these operators which will be needed later.

Section 8 is devoted to the proof of a positive (in the Krein sense) commutator estimate for the operators considered in Section 7. It relies on abstract conditions on the scalar operators $h, k$ appearing in (1.1).

In Section 9 resolvent estimates are proved for the generators $H$ on energy spaces. From them we deduce similar estimates for the quadratic pencils considered in Section 5, which in turn imply resolvent estimates for the generators $K$ on charge spaces.

Section 11 is devoted to the proof of propagation estimates for the groups $\mathrm{e}^{\mathrm{i} t H}$ and $\mathrm{e}^{\mathrm{i} t} K$. They follow from resolvent estimates by the standard arguments, usually applied in the Hilbert space setting.

In Section 12 we give various examples of our abstract class of Klein-Gordon equations. The first examples are Klein-Gordon equations on scattering manifolds, minimally coupled to external electro-magnetic fields. The massive and massless cases are discussed separately, a Hardy inequality playing an important role in the massless case. The second examples are Klein-Gordon equations on asymptotically hyperbolic manifolds, again with minimal coupling.

Various technical proofs are collected in Appendices A and B. 


\section{Some preparations}

In this section we collect some notation and preparatory material which will be used in later sections.

\subsection{Notations}

Sets

- If $X, Y$ are sets and $f: X \rightarrow Y$ we write

$$
f: X \stackrel{\sim}{\longrightarrow} Y
$$

if $f$ is bijective. If $X, Y$ are equipped with topologies, we use the same notation if $f: X \rightarrow Y$ is a homeomorphism.

- If $I \subset \mathbb{R}$ and $f$ is a real valued function defined on $I$ then $f(I)$ denotes the image of $I$ under $f$.

Examples of this notation used in Subsection 8.3 are $\sqrt{I}, I^{2}$ and $|I|$.

- We set

$$
\langle\lambda\rangle:=\left(\lambda^{2}+1\right)^{\frac{1}{2}}, \quad \lambda \in \mathbb{R} .
$$

LINEAR OPERATORS

- If $E \subset F$ are Banach spaces, we denote by $[E, F]_{\theta}, 0 \leq \theta \leq 1$ the complex interpolation space of order $\theta$.

- If $A$ is a closed, densely defined operator, we denote by $\rho(A) \subset \mathbb{C}$ its resolvent set and by $\operatorname{Dom} A$ its domain.

- Let $X, Y, Z$ be Banach spaces such that $X \subset Y \subset Z$ with continuous and dense embeddings. Then to each continuous operator $\hat{S}: X \stackrel{\sim}{\rightarrow} Z$ one may associate a densely defined operator $S$ acting in $Y$ defined as the restriction of $\hat{S}$ to the domain $\operatorname{Dom} S=(\widehat{S})^{-1}(Y)$.

- If $\mathcal{H}_{1}$ and $\mathcal{H}_{2}$ are Hilbert spaces, we denote by

$$
B_{\infty}\left(\mathcal{H}_{1}, \mathcal{H}_{2}\right)
$$

the ideal of compact operators from $\mathcal{H}_{1}$ to $\mathcal{H}_{2}$ and set $B_{\infty}(\mathcal{H})=B_{\infty}(\mathcal{H}, \mathcal{H})$. 
- If $a, b$ are linear operators, then we set

$$
\operatorname{ad}_{a}(b):=[a, b]
$$

Usually in this paper commutators are defined in the operator sense, i.e. $[a, b]$ has domain $\operatorname{Dom}(a b) \cap \operatorname{Dom}(b a)$.

- If $A, B$ are two positive self-adjoint operators on a Hilbert space $\mathcal{H}$, we write

$$
A \sim B
$$

if

$$
\operatorname{Dom} A^{\frac{1}{2}}=: \operatorname{Dom} B^{\frac{1}{2}} \text { and } c^{-1} A \leq B \leq c A \text { on } \operatorname{Dom} A^{\frac{1}{2}}, c>0
$$

\section{DUAL PAIRS}

Let $\mathcal{G}$ and $\mathcal{H}$ be reflexive Banach spaces and $\mathcal{E}=\mathcal{G} \oplus \mathcal{H}$. The usual realization $(\mathcal{G} \oplus \mathcal{H})^{*}=\mathcal{G}^{*} \oplus \mathcal{H}^{*}$ of the adjoint space will not be convenient in the sequel, we shall rather set

$$
\mathcal{E}^{*}:=\mathcal{H}^{*} \oplus \mathcal{G}^{*}
$$

so that

$$
\langle w \mid f\rangle=\left\langle w_{0} \mid f_{1}\right\rangle+\left\langle w_{1} \mid f_{0}\right\rangle, \quad \text { for } f=\left(f_{0}, f_{1}\right) \in \mathcal{E}, w=\left(w_{0}, w_{1}\right) \in \mathcal{E}^{*} .
$$

For example, if $\mathcal{H}=\mathcal{G}^{*}$, so $\mathcal{H}^{*}=\mathcal{G}$, the adjoint space of $\mathcal{E}=\mathcal{G} \oplus \mathcal{G}^{*}$ is identified with itself $\mathcal{E}^{*}=\mathcal{E}$.

\section{Scale of Sobolev SPaces}

Let $\mathcal{H}$ be a Hilbert space with norm $\|\cdot\|$ and scalar product $(\cdot \mid \cdot)$. We identify $\mathcal{H}$ with its adjoint space $\mathcal{H}^{*}=\mathcal{H}$ via the Riesz isomorphism. Let $h$ be a self-adjoint operator on $\mathcal{H}$.

We can associate to it the non-homogeneous Sobolev spaces

$$
\langle h\rangle^{-s} \mathcal{H}:=\operatorname{Dom}|h|^{s},\langle h\rangle^{s} \mathcal{H}:=\left(\langle h\rangle^{-s} \mathcal{H}\right)^{*}, \quad s \geq 0 .
$$

The spaces $\langle h\rangle^{-s} \mathcal{H}$ are equipped with the graph norm $\left\|\langle h\rangle^{s} u\right\|$.

If moreover Kerh $=\{0\}$, then we can also define the homogeneous Sobolev spaces $|h|^{s} \mathcal{H}$ equal to the completion of Dom $|h|^{-s}$ for the norm $\left\||h|^{-s} u\right\|$.

The notation $\langle h\rangle^{s} \mathcal{H}$ or $|h|^{s} \mathcal{H}$ is very convenient but somewhat ambiguous because usually $a \mathcal{H}$ denotes the image of $\mathcal{H}$ under the linear operator $a$. Let us explain how to reconcile these two meanings. 
Let $\mathcal{H}_{\mathrm{c}}$ be the space of $u \in \mathcal{H}$ such that $u=\mathbb{1}_{I}(h) u$, for some compact $I \subset \mathbb{R} \backslash\{0\}$. We equip $\mathcal{H}_{\mathrm{c}}$ with its natural topology by saying that $u_{n} \rightarrow u$ in $\mathcal{H}_{\mathrm{c}}$ if there exists $I \subset \mathbb{R} \backslash\{0\}$ compact such that $u_{n}=\mathbb{1}_{I}(h) u_{n}$ for all $n$ and $u_{n} \rightarrow u$ in $\mathcal{H}$. We set

$$
\mathcal{H}_{\text {loc }}:=\left(\mathcal{H}_{\mathrm{c}}\right)^{*} \text {. }
$$

Then $|h|^{s}$ and $\langle h\rangle^{s}$ preserve $\mathcal{H}_{\mathrm{c}}$ and $\mathcal{H}_{\text {loc }}$, and $\langle h\rangle^{s} \mathcal{H}$, resp. $|h|^{s} \mathcal{H}$ are the images in $\mathcal{H}_{\text {loc }}$ of $\mathcal{H}$ under $\langle h\rangle^{s}$, resp. $|h|^{s}$. It follows that these spaces are subspaces (equipped with finer topologies) of $\mathcal{H}_{\text {loc }}$, in particular they are pairwise compatible. Let us mention some properties of these spaces:

$$
\begin{gathered}
\langle h\rangle^{-s} \mathcal{H} \subset\langle h\rangle^{-t} \mathcal{H} \quad \text { if } t \leq s, \\
\langle h\rangle^{-s} \mathcal{H} \subset|h|^{-s} \mathcal{H} \quad \text { and } \quad|h|^{s} \mathcal{H} \subset\langle h\rangle^{s} \mathcal{H} \quad \text { if } s \geq 0, \\
\langle h\rangle^{0} \mathcal{H}=|h|^{0} \mathcal{H}=\mathcal{H}, \quad\langle h\rangle^{s} \mathcal{H}=\left(\langle h\rangle^{-s} \mathcal{H}\right)^{*}, \quad|h|^{s} \mathcal{H}=\left(|h|^{-s} \mathcal{H}\right)^{*}, \\
0 \in \rho(h) \Longleftrightarrow\langle h\rangle^{s} \mathcal{H}=|h|^{s} \mathcal{H} \text { for some } s \neq 0 \Longleftrightarrow\langle h\rangle^{s} \mathcal{H}=|h|^{s} \mathcal{H} \text { for all } s .
\end{gathered}
$$

Moreover the operator $|h|^{s}$ is unitary from $|h|^{-t} \mathcal{H}$ to $|h|^{s-t} \mathcal{H}$ for all $s, t \in \mathbb{R}$.

The following fact is a rephrasing of the Kato-Heinz theorem:

$$
\text { if } a \sim b \text { then } a^{s} \mathcal{H}=b^{s} \mathcal{H} \quad \text { for all }|s| \leq \frac{1}{2} .
$$

\section{SMOOTHNESS OF OPERATORS}

Let $\mathcal{H}_{1}, \mathcal{H}_{2}$ be two Banach spaces such that $\mathcal{H}_{1} \subset \mathcal{H}_{2}$ continuously and densely. Let $\left\{T_{t}\right\}_{t \in \mathbb{R}}$ be a $C_{0}$-group on $\mathcal{H}_{2}$, preserving $\mathcal{H}_{1}$. It follows by [1, Proposition 3.2.5] that $T_{t}$ defines a $C_{0}$-group on $\mathcal{H}_{1}$. If $a$ is the generator of $T_{t}$ on $\mathcal{H}_{2}$, so that $T_{t}=\mathrm{e}^{\mathrm{i} t a}$ on $\mathcal{H}_{2}$, then the generator of $T_{t}$ on $\mathcal{H}_{1}$ is $a_{\mid \mathcal{H}_{1}}$ with domain $\left\{u \in \mathcal{H}_{1} \cap \operatorname{Dom} a: a u \in \mathcal{H}_{1}\right\}$.

We denote by $C^{k}\left(a ; \mathcal{H}_{1}, \mathcal{H}_{2}\right)$ (resp. $\left.C_{\mathrm{u}}^{k}\left(a ; \mathcal{H}_{1}, \mathcal{H}_{2}\right)\right)$ for $k \in \mathbb{N}$ the space of operators $b \in B\left(\mathcal{H}_{1}, \mathcal{H}_{2}\right)$ such that

$$
\mathrm{R} \ni t \longmapsto \mathrm{e}^{\mathrm{i} t a} b \mathrm{e}^{-\mathrm{i} t a}
$$

is $C^{k}$ for the strong (resp. operator) topology of $B\left(\mathcal{H}_{1}, \mathcal{H}_{2}\right)$.

One defines similarly $C_{(\mathrm{u})}^{s}\left(a ; \mathcal{H}_{1}, \mathcal{H}_{2}\right)$ first for $0<s<1$, then for all non integers $s \in \mathbb{R}^{+}$by requiring the Hölder continuity of the above map. Note that by the uniform boundedness principle, the spaces $C^{s}\left(a ; \mathcal{H}_{1}, \mathcal{H}_{2}\right)$ and $C_{\mathrm{u}}^{s}\left(a ; \mathcal{H}_{1}, \mathcal{H}_{2}\right)$ coincide for non integer $s$. It follows also from the same argument that

$$
C^{k}\left(a ; \mathcal{H}_{1}, \mathcal{H}_{2}\right) \subset C_{\mathrm{u}}^{s}\left(a ; \mathcal{H}_{1}, \mathcal{H}_{2}\right), \quad \text { for } 0<s<k .
$$


If $b \in C^{1}\left(a ; \mathcal{H}_{1}, \mathcal{H}_{2}\right)$ then $b$ maps Dom $a_{\mid \mathcal{H}_{1}}$ into Dom $a$ and

$$
\operatorname{ad}_{a} b:=a b-b a \in B\left(\mathcal{H}_{1}, \mathcal{H}_{2}\right) .
$$

If $\mathcal{H}_{1}=\mathcal{H}_{2}=\mathcal{H}$, the above spaces are simply denoted by $C_{(\mathrm{u})}^{s}(a ; \mathcal{H})$ or even $C_{(\mathrm{u})}^{s}(a)$ if $\mathcal{H}$ is fixed from the context.

2.2. Quadratic pencils. In this subsection we prove some basic results about a quadratic operator pencil related to the abstract Klein-Gordon operator.

Let $\mathcal{H}$ be a Hilbert space, $h$ be a self-adjoint operator on $\mathcal{H}$ and $\langle h\rangle^{-s} \mathcal{H}$ the Sobolev spaces introduced in Subsection 2.1. Let

$$
k:\langle h\rangle^{-\frac{1}{2}} \mathcal{H} \longrightarrow \mathcal{H}
$$

be a continuous symmetric operator and denote also $k$ its unique extension to a continuous map $\mathcal{H} \rightarrow\langle h\rangle^{\frac{1}{2}} \mathcal{H}$. Denote

$$
h_{0}=h+k^{2}:\langle h\rangle^{-\frac{1}{2}} \mathcal{H} \longrightarrow\langle h\rangle^{\frac{1}{2}} \mathcal{H}
$$

and

$$
p(z)=h+z(2 k-z)=h_{0}-(k-z)^{2} \in B\left(\langle h\rangle^{-\frac{1}{2}} \mathcal{H},\langle h\rangle^{\frac{1}{2}} \mathcal{H}\right), \quad z \in \mathbb{C} .
$$

Definition 2.1. We denote by $\rho(h, k)$ the set of $z \in \mathbb{C}$ such that

$$
p(z):\langle h\rangle^{-\frac{1}{2}} \mathcal{H} \stackrel{\sim}{\longrightarrow}\langle h\rangle^{\frac{1}{2}} \mathcal{H} .
$$

Observe that the domain in $\mathcal{H}$ of the operator

$$
p(z):\langle h\rangle^{-\frac{1}{2}} \mathcal{H} \longrightarrow\langle h\rangle^{\frac{1}{2}} \mathcal{H}
$$

is equal to $\langle h\rangle^{-1} \mathcal{H}$, i.e.

$$
\langle h\rangle^{-1} \mathcal{H}=p(z)^{-1} \mathcal{H} .
$$

Indeed, for $f \in\langle h\rangle^{-\frac{1}{2}} \mathcal{H}$,

$$
p(z) f=h f+z(2 k-z) f
$$

and the last term belongs to $\mathcal{H}$, hence $p(z) f \in \mathcal{H}$ if and only if $h f \in \mathcal{H}$. Note also that the relation

$$
p(z)^{*}=p(\bar{z}) \quad \text { in } B\left(\langle h\rangle^{-\frac{1}{2}} \mathcal{H},\langle h\rangle^{\frac{1}{2}} \mathcal{H}\right)
$$

is obvious. It follows that the map

$$
p(z):\langle h\rangle^{-\frac{1}{2}} \mathcal{H} \longrightarrow\langle h\rangle^{\frac{1}{2}} \mathcal{H}
$$

naturally induces operators in $B\left(\langle h\rangle^{-1} \mathcal{H}, \mathcal{H}\right)$ and $B(\mathcal{H},\langle h\rangle \mathcal{H})$. 
The following two results are proved in $[9$, Lemmas 8.1, 8.2].

Lemma 2.2. The operator induced by $p(z)$ in $\mathcal{H}$ is a closed operator and its Hilbert space adjoint is the operator induced by $p(\bar{z})$ in $\mathcal{H}$. In other terms, the relation $p(z)^{*}=p(\bar{z})$ also holds in the sense of closed operators in $\mathcal{H}$. The following conditions are equivalent:

$$
\begin{aligned}
& p(z):\langle h\rangle^{-1} \mathcal{H} \stackrel{\sim}{\longrightarrow} \mathcal{H}, \\
& p(\bar{z}):\langle h\rangle^{-1} \mathcal{H} \stackrel{\sim}{\longrightarrow} \mathcal{H}, \\
& p(z):\langle h\rangle^{-\frac{1}{2}} \mathcal{H} \stackrel{\sim}{\longrightarrow}\langle h\rangle^{\frac{1}{2}} \mathcal{H}, \\
& p(\bar{z}):\langle h\rangle^{-\frac{1}{2}} \mathcal{H} \stackrel{\sim}{\longrightarrow}\langle h\rangle^{\frac{1}{2}} \mathcal{H}, \\
& p(z): \mathcal{H} \stackrel{\sim}{\longrightarrow}\langle h\rangle \mathcal{H}, \\
& p(\bar{z}): \mathcal{H} \stackrel{\sim}{\longrightarrow}\langle h\rangle \mathcal{H} .
\end{aligned}
$$

In particular, the set

$$
\begin{aligned}
\rho(h, k) & :=\left\{z \in \mathbb{C} \mid p(z):\langle h\rangle^{-\frac{1}{2}} \mathcal{H} \stackrel{\sim}{\longrightarrow}\langle h\rangle^{\frac{1}{2}} \mathcal{H}\right\} \\
& =\left\{z \in \mathbb{C} \mid p(z):\langle h\rangle^{-1} \mathcal{H} \stackrel{\sim}{\longrightarrow} \mathcal{H}\right\}
\end{aligned}
$$

is invariant under conjugation.

Proposition 2.3. Assume that $h$ is bounded below. Then there exists $c_{0}>0$ such that

$$
\left\{z:|\operatorname{Im} z|>|\operatorname{Re} z|+c_{0}\right\} \subset \rho(h, k) .
$$

\section{Operators on Krein spaces}

In this section we review some basic facts about Krein spaces and self-adjoint operators on Krein spaces. We refer the reader for more details to the survey paper [22], or to [11], [9]. We also describe the natural extension of the continuous functional calculus constructed in [9] to the Borel case.

3.1. Krein spaces. If $\mathcal{H}$ is a topological complex vector space, we denote by $\mathcal{H}^{\#}$ the space of continuous linear forms on $\mathcal{H}$ and by $\langle w, u\rangle$, for $u \in \mathcal{H}, w \in \mathcal{H}^{\#}$ the duality bracket between $\mathcal{H}$ and $\mathcal{H}^{\#}$. 
Definition 3.1. A Krein space $\mathcal{K}$ is a hilbertizable vector space equipped with a bounded hermitian form $\langle u \mid v\rangle$ non-degenerate in the sense that if $w \in \mathcal{K}^{\#}$ there exists a unique $u \in \mathcal{K}$ such that

$$
\langle u \mid v\rangle=\langle w, v\rangle, \quad v \in \mathcal{K} .
$$

If $\mathcal{K}_{1}$ is a subspace of $\mathcal{K}$, we denote by $\mathcal{K}_{1}^{\perp}$ the orthogonal of $\mathcal{K}_{1}$ for $\langle\cdot \mid \cdot\rangle$.

If we fix a scalar product $(\cdot \mid \cdot)$ on $\mathcal{K}$ endowing $\mathcal{K}$ with its hilbertizable topology, then by the Riesz theorem there exists a bounded, invertible self-adjoint operator $M$ such that

$$
\langle u \mid v\rangle=(u \mid M v), \quad u, v \in \mathcal{K} .
$$

Using the polar decomposition of $M, M=J|M|$ where $J=J^{*}, J^{2}=\mathbb{1}$, one can equip $\mathcal{K}$ with the equivalent scalar product

$$
(u \mid v)_{M}:=(u|| M \mid v),
$$

so that

$$
\langle u \mid v\rangle=(u \mid J v)_{M}, \quad u, v \in \mathcal{K} .
$$

Definition 3.2. A Krein space $(\mathcal{K},\langle\cdot \mid \cdot\rangle)$ is a Pontryagin space if either $\mathbb{1}_{\mathbb{R}^{-}}(J)$ or $\mathbb{1}_{\mathbb{R}^{+}}(J)$ has finite rank.

Clearly this definition is independent on the choice of the scalar product $(\cdot \mid \cdot)$.

Replacing $\langle\cdot \mid \cdot\rangle$ by $-\langle\cdot \mid \cdot\rangle$ we can assume that $\mathbb{1}_{\mathbb{R}^{-}}(J)$ has finite rank, which is the usual convention for Pontryagin spaces.

Let $A: \operatorname{Dom} A \rightarrow \mathcal{K}$ be a densely defined linear operator on the Krein space $\mathcal{K}$. The adjoint $A^{\dagger}$ of $A$ on $(\mathcal{K},\langle\cdot \mid \cdot\rangle)$ is defined as

$$
\begin{array}{r}
\operatorname{Dom} A^{\dagger}:=\left\{u \in \mathcal{K}: \text { there exists } f=: A^{\dagger} u\right. \text { such that } \\
\\
\langle f \mid v\rangle=\langle u \mid A v\rangle, \text { for all } v \in \operatorname{Dom} A\} .
\end{array}
$$

We will sometimes use the following easy fact: there is a constant $C>0$ such that

$$
C^{-1}\|A\| \leq\left\|A^{\dagger}\right\| \leq C\|A\|, A \in B(\mathcal{K}) .
$$

A densely defined operator $H$ is self-adjoint on $\mathcal{K}$ if $H=H^{\dagger}$. The following fact is often useful.

Lemma 3.3. Let $H$ be closed and densely defined on $\mathcal{K}$. Assume that for some $z \in \rho(H) \cap \overline{\rho(H)}$ one has $\left((H-z)^{-1}\right)^{\dagger}=(H-\bar{z})^{-1}$. Then $H=H^{\dagger}$. 
3.2. Definitizable operators on Krein spaces. Not much of interest can be said about self-adjoint operators on a Krein space, except for the trivial fact that $\rho(H)=\overline{\rho(H)}$. There is however a special class of self-adjoint operators, called definitizable, which admit a functional calculus close to the one of usual selfadjoint operators on a Hilbert space.

Definition 3.4. A self-adjoint operator $H$ is definitizable if

(1) $\rho(H) \neq \varnothing$ and

(2) there exists a real polynomial $p(\lambda)$ such that

$$
\langle u \mid p(H) u\rangle \geq 0, \quad \text { for all } u \in \operatorname{Dom} H^{k}, k:=\operatorname{deg} p .
$$

An operator $H$ on a Krein space $\mathcal{K}$ which is definitizable with an even definitizing polynomial will be called even-definitizable.

The following result is well known, see e.g. [19, Lemma 1].

Proposition 3.5. Let $H$ be definitizable. Then

(1) If $z \in \sigma(H) \backslash \mathbb{R}$ then $p(z)=0$ for each definitizing polynomial $p$;

(2) there is a definitizing polynomial $p$ such that $\sigma(H) \backslash \mathbb{R}$ is exactly the set of non-real zeroes of $p$;

(3) moreover, this $p$ may be chosen such that if $\lambda \notin \mathbb{R}$ is a zero of multiplicity $k$ of $p$ then $\lambda$ is an eigenvalue of $H$ of Riesz index $k$;

(4) the non-real spectrum of $H$ consists of a finite number of eigenvalues of finite Riesz index distributed symmetrically with respect to the real axis.

The usefulness of the notion of Pontryagin spaces comes from the following theorem (see [22]).

Theorem 3.6. A self-adjoint operator $H$ on a Pontryagin space is even-definitizable.

The following result is easy (see Langer [22]). If $\lambda$ is an isolated point of $\sigma(H)$ the Riesz spectral projection on $\lambda$ is

$$
E(\lambda, H):=\frac{1}{2 \mathrm{i} \pi} \oint_{C}(z-H)^{-1} d z
$$

where $C$ is a small curve in $\rho(H)$ surrounding $\lambda$. 
Proposition 3.7. Let $H$ be a definitizable self-adjoint operator and

$$
\mathbb{1}_{\mathrm{pp}}^{\mathrm{C}}(H)=\sum_{\lambda \in \sigma(H), \operatorname{Im} \lambda>0}(E(\lambda, H)+E(\bar{\lambda}, H)), \quad \mathcal{K}_{\mathrm{pp}}^{\mathbb{C}}:=\mathbb{1}_{\mathrm{pp}}^{\mathrm{C}}(H) \mathcal{K} .
$$

Then $\mathbb{1}_{\mathrm{pp}}^{\mathrm{C}}(H)$ is a projection, $\mathbb{1}_{\mathrm{pp}}^{\mathrm{C}}(H)=\left(\mathbb{1}_{\mathrm{pp}}^{\mathrm{C}}(H)\right)^{\dagger}$, hence $\mathcal{K}_{\mathrm{pp}}^{\mathrm{C}}$ is a Krein space and

$$
\mathcal{K}=\mathcal{K}_{\mathrm{pp}}^{\mathbb{C}} \oplus\left(\mathcal{K}_{\mathrm{pp}}^{\mathbb{C}}\right)^{\perp} .
$$

3.3. $C^{\alpha}$ functional calculus. In this subsection we recall some results of [9], extending earlier results of $[19,22]$ on the continuous functional calculus for definitizable operators. It turns out that a definitizable operator $H$ admits a functional calculus associated to the algebra of bounded continuous functions on $\mathbb{R}$ having an asymptotic expansion of a specific order at each critical point of $H$ (see Definition 3.11).

Let $\widehat{\mathbb{R}}=\mathbb{R} \cup\{\infty\}$ be the one point compactification of $\mathbb{R}$, so that $C(\widehat{\mathbb{R}})$ is identified with the set of continuous functions $\mathbb{R} \rightarrow \mathbb{C}$ having a finite limit at $\infty$.

We equip $\widehat{\mathbb{R}} \times \mathbb{N}$ with the order relation defined by $(\xi, s) \leq(\eta, t)$ if and only if $\xi=\eta$ and $s \leq t$. If $\omega=(\xi, s) \in \widehat{\mathbb{R}} \times \mathbb{N}$ we denote by $\chi_{\omega}$ the rational function equal to $(x-\xi)^{s}$ if $\xi \in \mathbb{R}$ and $x^{-s}$ if $\xi=\infty$. We set also $\rho_{\omega}=\chi_{\omega}^{-1}$.

Definition 3.8. Let $\omega=(\xi, s) \in \widehat{\mathbb{R}} \times \mathbb{N}$. We denote by $C^{\omega}(\widehat{\mathbb{R}})$ the space of functions $\varphi \in C(\widehat{\mathbb{R}})$ such that there is a polynomial $P$ with

$$
\varphi(x)= \begin{cases}P(x-\xi)+o\left(|x-\xi|^{s}\right), & \text { if } \xi \in \mathbb{R}, \\ P(1 / x)+o\left(|x|^{-s}\right), & \text { if } \xi=\infty .\end{cases}
$$

Clearly $C^{\mu}(\widehat{\mathbb{R}}) \subset C^{\omega}(\widehat{\mathbb{R}})$ if $\mu \geq \omega$. If $\varphi \in C^{\omega}(\widehat{\mathbb{R}})$ then the terms of degree $\leq s$ of $P$ are uniquely determined, hence there is a unique sequence of complex numbers $\left\{\delta_{\mu}(\varphi)\right\}_{\mu \leq \omega}$ such that the rational function

$$
T_{\omega}^{+} \varphi:=\sum_{\mu \leq \omega} \delta_{\mu}(\varphi) \chi_{\mu}
$$

satisfies

$$
\varphi(x)=T_{\omega}^{+} \varphi(x)+o\left(\left|\chi_{\omega}(x)\right|\right) .
$$

Set

$$
T_{\omega} \varphi:=\sum_{\mu<\omega} \delta_{\mu}(\varphi) \chi_{\mu}, \quad R_{\omega} \varphi:=\rho_{\omega}\left(\varphi-T_{\omega} \varphi\right)
$$

so that

$$
\varphi=T_{\omega} \varphi+\chi_{\omega} R_{\omega} \varphi
$$


Note that if $\omega=(\xi, 0)$ then $R_{\omega} \varphi=\varphi$. If follows that

$$
\|\varphi\|_{\omega}:=\sum_{\mu \leq \omega} \sup \left|R_{\mu} \varphi\right|
$$

is a norm on $C^{\omega}(\widehat{\mathbb{R}})$ dominating the sup norm.

An element $\omega \in \widehat{\mathbb{R}} \times \mathbb{N}$ may be seen as a function $\widehat{\mathbb{R}} \rightarrow \mathbb{N}$ with support containing at most one point. A function $\alpha: \widehat{\mathbb{R}} \rightarrow \mathbb{N}$ with finite support is called an order function. We write

$$
\omega \preceq \alpha
$$

if

$$
\omega=(\xi, s) \in \widehat{\mathbb{R}} \times \mathbb{N} \quad \text { and } \quad s \leq \alpha(\xi) .
$$

Then

$$
\omega \prec \alpha
$$

means

$$
\omega \preceq \alpha \quad \text { and } \quad s<\alpha(\xi) .
$$

To each definitizable operator one can associate a natural order function.

Definition 3.9. Let $H$ be a definitizable operator on $\mathcal{K}$.

(1) To each definitizing polynomial $p$ for $H$ we associate an order function $\beta$ as follows: if $\xi \in \mathbb{R}$ then $\beta(\xi)$ is the multiplicity of $\xi$ as zero of $p$ and $\beta(\infty)=0$ if $p$ is of even degree and $\beta(\infty)=1$ if $p$ is of odd degree.

(2) The order function $\alpha_{H}$ of $H$ is the infimum over all definitizing polynomials for $H$ of the above functions $\beta$.

If $\alpha$ is an order function, we set

$$
C^{\alpha}(\widehat{\mathbb{R}}):=\bigcap_{\omega \preceq \alpha} C^{\omega}(\widehat{\mathbb{R}})
$$

which, equipped with the norm $\|\varphi\|_{\alpha}:=\sup _{\omega \preceq \alpha}\|\varphi\|_{\omega}$, is a unital Banach *algebra for the usual operations.

The following theorem is shown in [9, Theorem 4.9]

Theorem 3.10. Let $H$ be a self-adjoint definitizable operator on the Krein space $\mathcal{H}$ with $\sigma(H) \subset \mathbb{R}$.Then there is a unique linear continuous map

$$
C^{\alpha_{H}}(\widehat{\mathbb{R}}) \ni \varphi \longmapsto \varphi(H) \in B(\mathcal{K})
$$

such that if $\varphi(\lambda)=(\lambda-z)^{-1}$ for $z \in \mathbb{C} \backslash \mathbb{R}$ then $\varphi(H)=(H-z)^{-1}$. This map is a morphism of unital $*$-algebras. 
Theorem 3.10 implies an optimal estimate of the resolvent of a definitizable operator. We first introduce some terminology.

Definition 3.11. We set

$$
\sigma_{\mathbb{C}}(H):=\sigma(H) \backslash \mathbb{R}
$$

and

$$
c(H):=\left\{\omega \in \widehat{\mathbb{R}}: \alpha_{H}(\xi) \neq 0\right\}
$$

The set $c(H)$ is called the set of critical points of $H$.

Let $H$ be a definitizable operator. Note that Definition 3.9 extends naturally to give an order function on $\widehat{\mathbb{C}}=\mathbb{C} \cup\{\infty\}$, still denoted by $\alpha_{H}$. The following result is proved in [9, Proposition 4.15].

Proposition 3.12. With the preceding notations, there exists $c>0$ such that

$$
\begin{aligned}
& c\left\|(H-z)^{-1}\right\| \\
& \quad \leq \sum_{\xi \in \sigma_{\mathbb{C}}(H)}|z-\xi|^{-\alpha_{H}(\xi)}+|\operatorname{Im} z|^{-1}\left(1+\sum_{\xi \in c(H) \cap \mathbb{R}}|z-\xi|^{-\alpha_{H}(\xi)}+|z|^{\alpha_{H}(\infty)}\right)
\end{aligned}
$$

for all $z \notin \sigma_{\mathbb{C}}(H) \cup \mathbb{R}$. Note that $\alpha_{H}(\infty)$ is either 0 or 1 .

We will use the following corollary of Proposition 3.12, giving estimates on $(H-z)^{-1}$ in a bounded region or in a conic neighborhood of infinity in $\mathbb{C} \backslash \mathbb{R}$.

Corollary 3.13. Let for $R, a, \delta>0$,

$$
U_{0}(R, a)=\{z \in \mathbb{C}: 0<|\operatorname{Im} z|<a,|\operatorname{Re} z| \leq R\},
$$

and

$$
U_{\infty}(R, \delta)=\{z \in \mathbb{C}: 0<|\operatorname{Im} z| \leq \delta|\operatorname{Re} z|,|\operatorname{Re} z| \geq R\}
$$

where $R$, a are chosen such that $\sigma_{\mathbb{C}}(H)$ does not intersect $U_{0}(R, a)$ and $U_{\infty}(R, \delta)$. Then there exists $C>0$ such that

$$
\left\|(H-z)^{-1}\right\| \leq \begin{cases}C|\operatorname{Im} z|^{-m-1} & \text { for } z \in U_{0}(R, a), \\ C\langle z\rangle^{\alpha_{H}(\infty)}|\operatorname{Im} z|^{-1} & \text { for } z \in U_{\infty}(R, \delta),\end{cases}
$$

where $m=\sup _{\xi \in \mathbb{R}} \alpha_{H}(\xi)$. 
It is sometimes convenient to have a concrete expression of $\varphi(H)$ if $\varphi \in$ $C_{0}^{\infty}(\mathbb{R})$. Let $\tilde{\varphi} \in C_{0}^{\infty}(\mathbb{C})$ be an almost-analytic extension of $\varphi$, satisfying

$$
\tilde{\varphi}_{\mid \mathbb{R}}=\varphi,\left|\frac{\partial \tilde{\varphi}(z)}{\partial \bar{z}}\right| \leq C_{N}|\operatorname{Imz}|^{N}, \quad \text { for all } N \in \mathbb{N} .
$$

For $m \in \mathbb{N}$ we set

$$
\|\varphi\|_{m}:=\sum_{0 \leq k \leq m}\left\|\partial_{x}^{k} \varphi\right\|_{\infty}
$$

Then we have

$$
\varphi(H)=\frac{\mathrm{i}}{2 \pi} \int_{\mathbb{C}} \frac{\partial \tilde{\varphi}}{\partial \bar{z}}(z)(z-H)^{-1} d z \wedge d \bar{z},
$$

where due to Corollary 3.13 the integral is norm-convergent and one has

$$
\|\varphi(H)\| \leq C\|\varphi\|_{m}, \quad \text { for some } m \in \mathbb{N} .
$$

3.4. Borel functional calculus. In this subsection we extend the results of Subsection 3.3 to cover the Borel functional calculus. Similar results were already obtained by Jonas [19], see also [36], although we believe that our approach is simpler and more transparent.

The standard method to obtain a Borel functional calculus from a continuous one relies on the Riesz and monotone class theorems (see Theorem B.1 and the beginning of the Appendix B for details).

In our case we have to follow the same procedure, starting from the algebra $C^{\alpha}(\widehat{\mathbb{R}})$ instead of $C(\widehat{\mathbb{R}})$. In turns out that the resulting algebra is not an algebra of functions on $\widehat{\mathbb{R}}$, because after a bounded limit, the top order term in the asymptotic expansion (3.7) is not uniquely determined. Instead the resulting algebra is a direct sum of a sub-algebra of bounded Borel functions satisfying (3.12) below, and of a finite dimensional space.

We first introduce some definitions. Denote by $B(\widehat{\mathbb{R}})$ the space of bounded Borel functions on $\widehat{\mathbb{R}}$.

Definition 3.14. Let $\omega=(\xi, s) \in \widehat{\mathbb{R}} \times \mathbb{N}$. We denote by $L^{\omega}(\widehat{\mathbb{R}})$ the space of functions $\varphi \in B(\widehat{\mathbb{R}})$ such that there is a polynomial $P$ with

$$
\varphi(x)= \begin{cases}P(x-\xi)+O\left(|x-\xi|^{s}\right) & \text { if } \xi \in \mathbb{R}, \\ P(1 / x)+O\left(|x|^{-s}\right) & \text { if } \xi=\infty .\end{cases}
$$


Again $L^{\mu}(\widehat{\mathbb{R}}) \subset L^{\omega}(\widehat{\mathbb{R}})$ if $\mu \geq \omega$. If $\varphi \in L^{\omega}(\widehat{\mathbb{R}})$ for $\omega=(\xi, s), s \geq 1$, the terms of degree $<s$ of $P$ are uniquely determined, hence there is a unique sequence $\left\{\delta_{\mu}(\varphi)\right\}_{\mu<\omega}$ such that the rational function $T_{\omega} \varphi$ defined in (3.8) satisfies

$$
\varphi(x)=T_{\omega} \varphi(x)+O\left(\left|\chi_{\omega}(x)\right|\right) .
$$

If $\omega=(\xi, 0)$ we set $\delta_{\omega}(\varphi):=\varphi(\xi)$. We equip $L^{\omega}(\widehat{\mathbb{R}})$ with the norm $\|\varphi\|_{\omega}$ as before and if $\alpha$ is an order function, we introduce the space

$$
L^{\alpha}(\widehat{\mathbb{R}}):=\bigcap_{\omega \leq \alpha} L^{\omega}(\widehat{\mathbb{R}})
$$

equipped with the norm $\|\varphi\|_{\alpha}$.

Clearly $L^{\alpha}(\widehat{\mathbb{R}})$ is a unital Banach $*$-algebra for the usual algebraic operations.

Definition 3.15. Let

$$
\tilde{\alpha}=\{(\xi, \alpha(\xi)): \xi \in \operatorname{supp} \alpha\} \subset \widehat{\mathbb{R}} \times \mathbb{N} .
$$

We set

$$
\Lambda^{\alpha}:=L^{\alpha}(\widehat{\mathbb{R}}) \oplus \mathbb{C}^{\tilde{\alpha}}
$$

and

$$
\begin{aligned}
I: C^{\alpha}(\widehat{\mathbb{R}}) & \longrightarrow \Lambda^{\alpha}, \\
\varphi & \longmapsto\left(\varphi,\left(\delta_{\omega}(\varphi)\right)_{\omega \in \tilde{\alpha}}\right) .
\end{aligned}
$$

For $\varphi=\left(\varphi^{0},\left(a_{\omega}\right)_{\omega \in \tilde{\alpha}}\right) \in \Lambda^{\alpha}$ and $\omega \preceq \alpha$, we define

$$
\delta_{\omega}(\varphi):= \begin{cases}\delta_{\omega}\left(\varphi^{0}\right) & \text { if } \omega \notin \tilde{\alpha}, \\ a_{\omega} & \text { if } \omega \in \tilde{\alpha},\end{cases}
$$

which allows to write $\varphi$ as $\left(\varphi^{0},\left(\delta_{\omega}(\varphi)\right)_{\omega \in \tilde{\alpha}}\right)$. We can then equip $\Lambda^{\alpha}$ with a $*$-algebra structure by setting

$$
\begin{aligned}
\varphi \psi & :=\left(\varphi^{\circ},\left(\delta_{\omega}(\varphi)\right)_{\omega \in \tilde{\alpha}}\right) \cdot\left(\psi^{\circ},\left(\delta_{\omega}(\psi)\right)_{\omega \in \tilde{\alpha}}\right) \\
& =\left(\varphi^{\circ} \psi^{\circ},\left(\sum_{\mu+\nu=\omega} \delta_{\mu}(\varphi) \delta_{\nu}(\psi)\right)_{\omega \in \tilde{\alpha}}\right),
\end{aligned}
$$

and

$$
\left.\left(\varphi^{0},\left(\delta_{\omega}(\varphi)\right)_{\omega \in \tilde{\alpha}}\right)^{*}:=\overline{\left(\varphi^{0}\right.},\left(\overline{\delta_{\omega}}(\varphi)\right)_{\omega \in \tilde{\alpha}}\right) .
$$

It is easy to see that $\Lambda^{\alpha}$, equipped with the norm

$$
\|\varphi\|_{\Lambda_{\alpha}}=\max _{\omega \leq \alpha} \max \left\{\left\|\varphi^{\circ}\right\|_{\omega}, \sum_{\mu \leq \omega}\left|\delta_{\mu}(\varphi)\right|\right\}
$$

is a unital Banach $*$-algebra with $(1,0)$ as unit. The embedding $I: C^{\alpha}(\widehat{\mathbb{R}}) \rightarrow \Lambda^{\alpha}$ is isometric hence $C^{\alpha}(\widehat{\mathbb{R}})$ is identified with a closed $*$-subalgebra of $\Lambda^{\alpha}$. 
Definition 3.16. A sequence $\left(\varphi_{n}\right)_{n \in \mathbb{N}}$ in $\Lambda^{\alpha}$ is $b$-convergent to $\varphi$ if

$$
\sup _{n}\left\|\varphi_{n}\right\|_{\Lambda^{\alpha}}<\infty \quad \text { and } \quad \lim _{n} \delta_{\omega}\left(\varphi_{n}\right)=\delta_{\omega}(\varphi)
$$

for each $\omega \preceq \alpha$.

Clearly the b-convergence of $\left(\varphi_{n}\right)$ to $\varphi$ implies the b-convergence of $\left(\varphi_{n}^{0}\right)$ to $\varphi^{0}$.

The main result of this subsection is the following theorem which is the natural extension of Theorem 3.10 to the Borel case.

Theorem 3.17. Let $H$ be a self-adjoint definitizable operator on a Krein space $\mathcal{K}$ with $\sigma(H) \subset \mathbb{R}$ and order function $\alpha_{H}$. Then there is a unique linear weakly b-continuous map

$$
\Lambda^{\alpha_{H}} \ni \varphi \longmapsto \varphi(H) \in B(\mathcal{K})
$$

such that if $\varphi=I r_{z}$, with $r_{z}(\lambda)=(\lambda-z)^{-1}$ and $z \in \mathbb{C} \backslash \mathbb{R}$, then

$$
\varphi(H)=(H-z)^{-1} .
$$

This map is a norm continuous morphism of unital $*$-algebras.

The proof will be given in Appendix B.

Corollary 3.18. Let $H$ a self-adjoint definitizable operator as above. Let $J \subset \widehat{\mathbb{R}}$ an open set such that $\bar{J} \cap \operatorname{supp} \alpha_{H}=\emptyset$ and $B_{J}(\widehat{\mathbb{R}}) \subset B(\widehat{\mathbb{R}})$ be the $*$-ideal of functions supported in $\bar{J}$. Then the map

$$
B_{J}(\widehat{\mathbb{R}}) \ni \varphi \longmapsto \varphi(H):=(\varphi, 0)(H) \in B(\mathcal{K})
$$

is a *-morphism, continuous for the norm topologies of $B_{J}(\widehat{\mathbb{R}})$ and $B(\mathcal{K})$ and weakly b-continuous.

Proof. Let us denote by $C_{J}(\widehat{\mathbb{R}}) \subset C(\widehat{\mathbb{R}})$ the $*$-ideal of functions supported in $\bar{J}$. Clearly $C_{J}(\widehat{\mathbb{R}}) \subset C^{\alpha_{H}}(\widehat{\mathbb{R}})$ isometrically. Moreover $I \varphi=(\varphi, 0)$ for all $\varphi \in C_{J}(\widehat{\mathbb{R}})$, if $I: C^{\alpha_{H}}(\widehat{\mathbb{R}}) \rightarrow \Lambda^{\alpha_{H}}$ is defined in Definition 3.15. Finally if $\varphi_{n} \in B_{J}(\widehat{\mathbb{R}})$ and $\mathrm{b}-\lim _{n} \varphi_{n}=\varphi$ then $\mathrm{b}-\lim _{n}\left(\varphi_{n}, 0\right)=(\varphi, 0) \in \Lambda^{\alpha}$. These facts imply the corollary. 
3.5. Existence of the dynamics. Let us mention a well-known consequence of Corollary 3.18 about the existence of the dynamics generated by an even-definitizable operator.

Let $H$ be even-definitizable, $f_{t}: x \mapsto \mathrm{e}^{\mathrm{i} t x}$ and $\chi \in C_{0}^{\infty}(\mathbb{R})$ such all finite critical points of $H$ are in the support of $\chi$. We write $f_{t}=\chi f_{t}+(1-\chi) f_{t}$, and extend $(1-\chi) f_{t}$ arbitrarily at $\infty$. We can define $\left(\chi f_{t}\right)(H)$ by Theorem 3.10 and $\left((1-\chi) f_{t}\right)(H)$ by Corollary 3.18. We set then

$$
f_{t}(H):=\left(\chi f_{t}\right)(H)+\left((1-\chi) f_{t}\right)(H) \in B(\mathcal{K}),
$$

which is independent on the choice of $\chi$ with the above properties.

The space $\mathcal{K}_{\mathrm{pp}}^{\mathrm{C}}=\mathbb{1}_{\mathrm{pp}}^{\mathrm{C}}(H) \mathcal{K}$ is finite dimensional and invariant under $H$, hence we can obviously define $\left(\mathrm{e}^{\mathrm{i} t H}\right)_{\mid \mathcal{K}_{\mathrm{pp}}^{\mathrm{C}}}$. We then set

$$
\mathrm{e}^{\mathrm{i} t H}:=f_{t}(H)+\left(\mathrm{e}^{\mathrm{i} t H}\right)_{\mid \mathcal{K}_{\mathrm{pp}}^{\mathrm{C}}}, t \in \mathbb{R} .
$$

It is easy to see that $\left\{\mathrm{e}^{\mathrm{i} t H}\right\}_{t \in \mathbb{R}}$ is a $C_{0}$-group on $\mathcal{K}$, with $\left(\mathrm{e}^{\mathrm{i} t H}\right)^{\dagger}=\mathrm{e}^{-\mathrm{i} t H}$, i.e. a unitary $C_{0}$-group on $(\mathcal{K},\langle\cdot \mid \cdot\rangle)$. Moreover $H$ is the generator of $\left\{\mathrm{e}^{\mathrm{i} t H}\right\}_{t \in \mathbb{R}}$ and there exist $C, \lambda>0, n \in \mathbb{N}$ such that

$$
\left\|\left(\mathrm{e}^{\mathrm{i} t H}\right)_{\mid \mathcal{K}_{\mathrm{pp}}^{\mathrm{C}}}\right\| \leq C \mathrm{e}^{\lambda|t|},\left\|\left(\mathrm{e}^{\mathrm{i} t H}\right)_{\mid\left(\mathcal{K}_{\mathrm{pp}}^{\mathrm{C}}\right) \perp}\right\| \leq C\langle t\rangle^{n}, \quad t \in \mathbb{R} .
$$

\section{Abstract Klein-Gordon equations}

Let us discuss in more details the Klein-Gordon equation (1.1). The scalar product on $\mathcal{H}$ will be denoted by $(u \mid v)$ or sometimes by $\bar{u} \cdot v$.

To associate a generator to (1.1) one has to turn this equation into a first order evolution equation. It turns out that there are several ways to do this, leading to different generators, and different topological spaces of Cauchy data.

In order to present the results of this paper, we first discuss these questions in an informal way, without worrying about the problems of existence, uniqueness or even the meaning of solutions to (1.1).

4.1. Symplectic setup. The most natural approach is to consider $y=\mathcal{H} \oplus \mathcal{H}$ whose elements are denoted by $(\varphi, \pi)$, and to equip it with the complex symplectic form (i.e. sesquilinear, non-degenerate, anti-hermitian)

$$
\overline{\left(\varphi_{1}, \pi_{1}\right)} \omega\left(\varphi_{2}, \pi_{2}\right):=\overline{\pi_{1}} \cdot \varphi_{2}-\overline{\varphi_{1}} \cdot \pi_{2} .
$$

The classical Hamiltonian is

$$
E(\varphi, \pi):=\overline{(\pi+\mathrm{i} k \varphi)} \cdot(\pi+\mathrm{i} k \varphi)+\bar{\varphi} \cdot h \varphi .
$$


We consider $\omega, E$ as maps from $y$ to $y^{*}$, where $y^{*}$ is the space of anti-linear forms on $y$ and set

$$
A:=-\mathrm{i} \omega^{-1} E=\left(\begin{array}{cc}
k & -\mathrm{i} \\
\mathrm{i} h_{0} & k
\end{array}\right),
$$

for $h_{0}=h+k^{2}$. In other words $\mathrm{e}^{\mathrm{i} t A}$ is the symplectic flow obtained from the classical Hamiltonian $E$.

If we set

$$
\left(\begin{array}{c}
\varphi(t) \\
\pi(t)
\end{array}\right):=\mathrm{e}^{\mathrm{i} t A}\left(\begin{array}{l}
\varphi \\
\pi
\end{array}\right)
$$

then

$$
\phi(t):=\varphi(t)
$$

solves the Cauchy problem

$$
\left\{\begin{array}{l}
\partial_{t}^{2} \phi(t)-2 \mathrm{i} k \partial_{t} \phi(t)+h \phi(t)=0, \\
\phi(0)=\varphi, \partial_{t} \phi(0)=\pi+\mathrm{i} k \varphi .
\end{array}\right.
$$

4.2. Quadratic pencils and stationary solutions. If we look for a solution of equation (1.1) of the form $\phi(t)=\mathrm{e}^{\mathrm{i} t z} \phi$ (or equivalently set $\mathrm{i}^{-1} \partial_{t}=z$ ), we obtain that $\phi$ should solve

$$
p(z) \phi=0, \text { for } p(z)=h_{0}-(k-z)^{2} .
$$

The map $z \mapsto p(z)$, called a quadratic pencil, is further discussed in Subsection 2.2 .

4.3. Charge setup. Since we work on a complex symplectic space, it is more convenient to turn the symplectic form $\omega$ into a hermitian form. In fact setting

$$
f:=\left[\begin{array}{c}
\varphi \\
\mathrm{i}^{-1} \pi
\end{array}\right]=\left[\begin{array}{l}
f_{0} \\
f_{1}
\end{array}\right]
$$

the hermitian form

$$
q:=\mathrm{i} \omega
$$

called the charge, takes the form

$$
\bar{f} q f=\left(f_{1} \mid f_{0}\right)+\left(f_{0} \mid f_{1}\right),
$$

and the energy $E$ becomes

$$
E(f, f)=\left\|f_{1}+k f_{0}\right\|^{2}+\left(f_{0} \mid h f_{0}\right) .
$$


Note that from (4.15) we obtain

$$
E(f, f)=\bar{f} q K f
$$

If

$$
f(t):=\mathrm{e}^{\mathrm{i} t K} f, \text { for } K:=\left(\begin{array}{cc}
k & \mathbb{1} \\
h_{0} & k
\end{array}\right),
$$

then $\phi(t)=f_{0}(t)$ solves the Cauchy problem

$$
\left\{\begin{array}{l}
\partial_{t}^{2} \phi(t)-2 \mathrm{i} k \partial_{t} \phi(t)+h \phi(t)=0, \\
\phi(0)=f_{0}, \mathrm{i}^{-1} \partial_{t} \phi(0)-k \phi(0)=f_{1} .
\end{array}\right.
$$

4.4. PDE setup. Finally let us describe the standard setup used in partial differential equations. We set

$$
f(t)=\mathrm{e}^{\mathrm{i} t H} f, \text { for } H:=\left(\begin{array}{cc}
0 & \mathbb{1} \\
h & 2 k
\end{array}\right),
$$

and $\phi(t)=f_{0}(t)$ solves the Cauchy problem

$$
\left\{\begin{array}{l}
\partial_{t}^{2} \phi(t)-2 \mathrm{i} k \partial_{t} \phi(t)+h \phi(t)=0, \\
\phi(0)=f_{0}, \mathrm{i}^{-1} \partial_{t} \phi(0)=f_{1} .
\end{array}\right.
$$

The charge and energy become

$$
\begin{aligned}
\bar{f} q f & =\left(f_{1} \mid f_{0}\right)+\left(f_{0} \mid f_{1}\right)-2\left(f_{0} \mid k f_{0}\right), \\
E(f, f) & =\left\|f_{1}\right\|^{2}+\left(f_{0} \mid h f_{0}\right) .
\end{aligned}
$$

Note that if

$$
\Phi=\left(\begin{array}{ll}
\mathbb{1} & 0 \\
k & \mathbb{1}
\end{array}\right)
$$

then $H \Phi=\Phi K$.

4.5. The choice of functional spaces. Let us now discuss the choice of the possible topologies to put on the spaces of Cauchy data. We will use the abstract Sobolev spaces $\langle h\rangle^{s} \mathcal{H}$ and $|h|^{s} \mathcal{H}$ associated to the self-adjoint operator $h$, whose definition and properties are given in Subsection 2.1. 
The first natural choices correspond to topologies for which the symplectic form $\omega$ is bounded. Note that our choice of $y=\mathcal{H} \oplus \mathcal{H}$ as symplectic space in Subsection 4.1 was quite arbitrary. In fact we can choose a reflexive Banach space $\mathcal{G}$ and set $y=\mathcal{G} \oplus \mathcal{G}^{*}$ equipped with

$$
\bar{g} \omega f:=\left\langle g_{1} \mid f_{0}\right\rangle-\left\langle g_{0} \mid f_{1}\right\rangle,
$$

where $\left\langle g_{0} \mid f_{1}\right\rangle=f_{1}\left(g_{0}\right)$ and $\left\langle g_{1} \mid f_{0}\right\rangle=\overline{\left\langle f_{0} \mid g_{1}\right\rangle}$. Clearly $\omega$ is sesquilinear, anti-hermitian, non degenerate and bounded on $y$.

Examples of such symplectic spaces are the charge spaces

$$
\mathcal{K}_{\theta}=\langle h\rangle^{-\theta} \mathcal{H} \oplus\langle h\rangle^{\theta} \mathcal{H}, \quad \dot{\mathcal{K}}_{\theta}=|h|^{-\theta} \mathcal{H} \oplus|h|^{\theta} \mathcal{H}, \quad \theta \geq 0 .
$$

In this case it is convenient to use the charge setup. An additional requirement is of course that $K$ should be well defined as a closed operator on $\mathcal{K}_{\theta}$ or $\dot{\mathcal{K}}_{\theta}$, possibly with non-empty resolvent set, and that $K$ be the generator of a strongly continuous group $\mathrm{e}^{\mathrm{i} t K}$.

Another possibility often used in partial differential equations is to forget about the symplectic form and consider instead spaces on which the energy $E$ is bounded. It is then more convenient to use the PDE setup, and to work with the generator $H$. Reasonable choices are then the energy spaces

$$
\mathcal{E}=\langle h\rangle^{-\frac{1}{2}} \mathcal{H} \oplus \mathcal{H}, \quad \dot{\mathcal{E}}=|h|^{-\frac{1}{2}} \mathcal{H} \oplus \mathcal{H} .
$$

To select convenient spaces among all these, it suffices to consider the 'static' Klein-Gordon equation

$$
\partial_{t}^{2} \phi(t)+\epsilon^{2} \phi(t)=0
$$

corresponding to $h=\epsilon^{2}, k=0$ (we assume of course that $\epsilon \geq 0$ is unbounded). In this case we have

$$
H=K=\left(\begin{array}{cc}
0 & \mathbb{1} \\
\epsilon^{2} & 0
\end{array}\right)=: H_{0} .
$$

On any space of Cauchy data, the group $\mathrm{e}^{\mathrm{i} t H_{0}}$ will be formally given by

$$
\mathrm{e}^{\mathrm{i} t H_{0}}=\left(\begin{array}{cc}
\cos (\epsilon t) & \mathrm{i} \epsilon^{-1} \sin (\epsilon t) \\
\mathrm{i} \epsilon \sin (\epsilon t) & \cos \epsilon t
\end{array}\right) .
$$

We see that among these spaces the only ones on which $\mathrm{e}^{\mathrm{i} t H_{0}}$ is bounded are $\dot{\mathcal{E}}$, $\mathcal{E}, \dot{\mathcal{K}}_{\frac{1}{4}}$ and $\mathcal{K}_{\frac{1}{4}}$. The first two are the usual homogeneous and non-homogeneous energy spaces. The last two are called the homogeneous and non-homogeneous charge spaces. Note that the space $\dot{\mathcal{K}}_{\frac{1}{4}}$ appears naturally as the one-particle space in the Fock quantization of (4.17).

In this paper we consider the two operators $H$ acting on $\mathcal{E}$ and $K$ acting on $\mathcal{K}_{\frac{1}{4}}$. 


\section{Klein-Gordon operators in energy spaces}

In this section we discuss the properties of the operator $H$ in Subsection 4.4 considered as acting on the energy spaces $\mathcal{E}$ or $\dot{\mathcal{E}}$.

5.1. Non-homogeneous energy space. Let us fix a self-adjoint operator $h$ on $\mathcal{H}$ and a bounded, symmetric operator $k:\langle h\rangle^{-\frac{1}{2}} \mathcal{H} \rightarrow \mathcal{H}$ as in Subsection 2.2.

The energy space $\mathcal{E}$ and its adjoint space $\mathcal{E}^{*}$ are defined by

$$
\mathcal{E}:=\langle h\rangle^{-\frac{1}{2}} \mathcal{H} \oplus \mathcal{H} \quad \text { and } \quad \mathcal{E}^{*}:=\mathcal{H} \oplus\langle h\rangle^{\frac{1}{2}} \mathcal{H},
$$

where we used the convention explained in Subsection 2.1. We have a continuous and dense embedding $\mathcal{E} \subset \mathcal{E}^{*}$.

Lemma 5.1. (1) $h:\langle h\rangle^{-\frac{1}{2}} \mathcal{H} \stackrel{\sim}{\rightarrow}\langle h\rangle^{\frac{1}{2}} \mathcal{H}$ if and only if $0 \in \rho(h)$ if and only if $0 \in \rho(h, k)$.

(2) If $0 \in \rho(h)$ then $\mathcal{E}$ equipped with the hermitian sesquilinear form

$$
\langle f \mid f\rangle_{\varepsilon}:=\left(f_{0} \mid h f_{0}\right)+\left(f_{1} \mid f_{1}\right)
$$

is a Krein space.

(3) If, in addition, $\operatorname{Tr} \mathbb{1}_{]-\infty, 0]}(h)<\infty$, then $\left(\mathcal{E},\langle\cdot \mid \cdot\rangle_{\mathcal{E}}\right)$ is Pontryagin.

Proof. (1) follows from Lemma 2.2. (2) and (3) are immediate.

5.2. Klein-Gordon operators on energy space. We set

$$
\widehat{H}:=\left(\begin{array}{cc}
0 & \mathbb{1} \\
h & 2 k
\end{array}\right) \in B\left(\mathcal{E}, \mathcal{E}^{*}\right) .
$$

Definition 5.2. The energy Klein-Gordon operator is the operator $H$ induced by $\hat{H}$ in $\mathcal{E}$. Its domain is given by

$$
\text { Dom } H:=\mathcal{D}=\langle h\rangle^{-1} \mathcal{H} \oplus\langle h\rangle^{-\frac{1}{2}} \mathcal{H}=(\widehat{H}-z)^{-1} \mathcal{E}, \quad z \in \rho(h, k) .
$$

We have

$$
H=\left(\begin{array}{cc}
0 & 1 \\
h & 2 k
\end{array}\right)
$$


Proposition 5.3. (1) One has $\rho(H)=\rho(h, k)$.

(2) In particular, if $\rho(h, k) \neq \emptyset$, then $H$ is a closed densely defined operator in $\mathcal{E}$ and its spectrum is invariant under complex conjugation.

(3) If $z \in \rho(h, k)$ then

$$
(H-z)^{-1}=p(z)^{-1}\left(\begin{array}{cc}
z-2 k & 1 \\
h & z
\end{array}\right) .
$$

Proof. We will prove (1) and (3). Note that (2) will follow then from Lemma 2.2.

Let $z \in \rho(H)$. If $f_{0} \in\langle h\rangle^{-1} \mathcal{H}$ with $p(z) f_{0}=0$, then $f=\left(f_{0}, z f_{0}\right) \in$ $\operatorname{Ker}(H-z)$; hence $f_{0}=0$. If $g_{1} \in \mathcal{H}$, then $g=\left(0, g_{1}\right) \in \mathcal{E}$ and if $f=(H-z)^{-1} g$ then $p(z) f_{0}=g_{1}$, hence

$$
p(z):\langle h\rangle^{-1} \mathcal{H} \stackrel{\sim}{\longrightarrow} \mathcal{H}
$$

and $z \in \rho(h, k)$. Therefore $\rho(H) \subset \rho(h, k)$.

Conversely let $z \in \rho(h, k)$ so that $p(z):\langle h\rangle^{-1} \mathcal{H} \stackrel{\sim}{\rightarrow} \mathcal{H}$. We shall show that $z \in \rho(H)$ and

$$
(H-z)^{-1}=\left(\begin{array}{cc}
\ell w & \ell \\
\ell h & z \ell
\end{array}\right), \quad \ell=p(z)^{-1}, w=z-2 k,
$$

completing the proof of (1) and (3). One must interpret carefully the operators appearing in the matrix above because $(H-z)^{-1}$ must send $\mathcal{E}$ into $\mathcal{D}$. More precisely, since $h f_{0} \in\langle h\rangle^{\frac{1}{2}} \mathcal{H}$ if $f_{0} \in\langle h\rangle^{-\frac{1}{2}} \mathcal{H}$, the factor $\ell$ in the product $\ell h$ is not the inverse of $p(z):\langle h\rangle^{-1} \mathcal{H} \stackrel{\sim}{\rightarrow} \mathcal{H}$ but of its extension

$$
p(z):\langle h\rangle^{-\frac{1}{2}} \mathcal{H} \stackrel{\sim}{\longrightarrow}\langle h\rangle^{\frac{1}{2}} \mathcal{H} .
$$

We can do this thanks to Lemma 2.2. Now a mechanical computation implies

$$
\begin{aligned}
& \left(\begin{array}{cc}
\ell w & \ell \\
\ell h & z \ell
\end{array}\right)\left(\begin{array}{cc}
-z & 1 \\
h & 2 k-z
\end{array}\right)\left(\begin{array}{l}
f_{0} \\
f_{1}
\end{array}\right) \\
& =\left(\begin{array}{cc}
\ell w & \ell \\
\ell h & z \ell
\end{array}\right)\left(\begin{array}{l}
-z f_{0}+f_{1} \\
h f_{0}-w f_{1}
\end{array}\right) \\
& =\left(\begin{array}{l}
f_{0} \\
f_{1}
\end{array}\right),
\end{aligned}
$$

for all $f=\left(f_{0}, f_{1}\right) \in \mathcal{D}$. 
Similarly for $g=\left(g_{0}, g_{1}\right) \in \mathcal{E}$ we compute

$$
\begin{aligned}
& \left(\begin{array}{cc}
-z & 1 \\
h & 2 k-z
\end{array}\right)\left(\begin{array}{cc}
\ell w & \ell \\
\ell h & z \ell
\end{array}\right)\left(\begin{array}{l}
g_{0} \\
g_{1}
\end{array}\right) \\
& =\left(\begin{array}{cc}
-z & 1 \\
h & -w
\end{array}\right)\left(\begin{array}{l}
\ell w g_{0}+\ell g_{1} \\
\ell h g_{0}+z \ell g_{1}
\end{array}\right) \\
& =\left(\begin{array}{l}
g_{0} \\
g_{1}
\end{array}\right),
\end{aligned}
$$

which holds because $h \ell w=w \ell h$ on $\langle h\rangle^{-\frac{1}{2}} \mathcal{H}$, where $\ell$ is the inverse of the map $p(z):\langle h\rangle^{-\frac{1}{2}} \mathcal{H} \rightarrow\langle h\rangle^{\frac{1}{2}} \mathcal{H}$. Thus $z \in \rho(H)$ and $(H-z)^{-1}$ is given by (5.5).

Theorem 5.4. Assume that $0 \in \rho(h)$.

(1) $H$ is a self-adjoint operator on the Krein space $\left(\mathcal{E},\langle\cdot \mid \cdot\rangle_{\mathcal{E}}\right)$ with $\rho(H) \neq \emptyset$.

(2) If in addition $\operatorname{Tr} \mathbb{1}_{-\infty, 0]}(h)<\infty$, then $H$ is even-definitizable.

Proof. If $0 \in \rho(h)$ then $0 \in \rho(h, k)=\rho(H)$ and from (5.5) we get

$$
H^{-1}=\left(\begin{array}{cc}
-2 h^{-1} k & h^{-1} \\
1 & 0
\end{array}\right) \text {. }
$$

By Lemma 3.3 it suffices to show that $\left(H^{-1}\right)^{\dagger}=H^{-1}$, which is a simple computation. This proves (1). Since any self-adjoint operator with non-empty resolvent set on a Pontryagin space is even-definitizable, (2) follows from Lemma 5.1.

Remark 5.5. We make some comments on the relationship between our framework in Section 5 and the one used by Langer, Najman, and Tretter in [23], devoted to abstract Klein-Gordon operators on energy spaces. The main difference with respect to [23] come from the fact that our conditions are stated in terms of $h$ instead of $h_{0}$. One argument in favor of using $h$ is that $h$ appears naturally in the definition of the Krein scalar product $\langle\cdot \mid \cdot\rangle_{\varepsilon}$. On the other hand, in many concrete situations, like the charged Klein-Gordon equation on Minkowski space, where $h_{0}=-\Delta+m^{2}$ and $k$ is a real potential, it is also natural to view $h$ as a perturbation of $h_{0}$ and to use $h_{0}$ as the reference operator.

In [23] it is assumed that $h_{0} \geq c_{0}>0$ (in particular, the wave equation is not considered) and that $k$ is a symmetric operator whose domain contains $\left\langle h_{0}\right\rangle^{-1 / 2} \mathcal{H}$. Other conditions are needed to get deeper facts, e.g. in [23] it is required that $1-h_{0}^{-1 / 2} k^{2} h_{0}^{-1 / 2}$ be a boundedly invertible operator on $\mathcal{H}$ to get a convenient definition of $h$. In our setting, $h$ is given (with no assumptions on its spectrum) and we require $\langle h\rangle^{-1 / 2} \mathcal{H} \subset D(k)$. 
Suppose that both $h_{0}$ and $h$ are bounded from below. Then it is easy to see that $\langle h\rangle^{-1 / 2} \mathcal{H}=\left\langle h_{0}\right\rangle^{-1 / 2} \mathcal{H}$ and the results of this section and of Section 7 are essentially equivalent to those in [23]. However, the operator $h$ in [23] could be unbounded from below and then there is no straightforward relation between their results and ours.

5.3. Homogeneous energy space. Assume that $\operatorname{Ker} h=\{0\}$. Then we can introduce the homogeneous energy space

$$
\dot{\mathcal{E}}:=|h|^{-\frac{1}{2}} \mathcal{H} \oplus \mathcal{H},
$$

equipped with his canonical Hilbert space structure. Note that $\mathcal{E} \subset \dot{\varepsilon}$ continuously and densely. Of course $\mathcal{E}=\dot{\varepsilon}$ if and only if $0 \in \rho(h)$, so the typical situation considered in the sequel is $0 \in \sigma(h)$.

The following analog of Lemma 5.1 is obvious.

Lemma 5.6. Assume that $\operatorname{Ker} h=\{0\}$. Then $\dot{\mathcal{E}}$ equipped with $\langle\cdot \mid \cdot\rangle_{\mathcal{E}}$ is a Krein space. If in addition $\operatorname{Tr} \mathbb{1}_{]-\infty, 0]}(h)<\infty$, then $\dot{\mathcal{E}}$ is Pontryagin.

\subsection{Klein-Gordon operators on homogeneous energy space.}

Definition 5.7. The (homogeneous) energy Klein-Gordon operator is the operator $\dot{H}$ induced by $\hat{H}$ in $\dot{\mathcal{E}}$. Its domain is given by

$$
\dot{\mathcal{D}}=\left(|h|^{-\frac{1}{2}} \mathcal{H} \cap|h|^{-1} \mathcal{H}\right) \oplus\langle h\rangle^{-\frac{1}{2}} \mathcal{H}=\{f \in \dot{\mathcal{E}}: \widehat{H} f \in \dot{\mathcal{E}}\}
$$

which is continuously and densely embedded in $\dot{\varepsilon}$. We have

$$
\dot{H}=\left(\begin{array}{cc}
0 & 1 \\
h & 2 k
\end{array}\right)
$$

Since $\mathcal{E} \subset \dot{\mathcal{E}}$ and $\mathcal{D} \subset \dot{\mathcal{D}}$ continuously and densely, $H$ may also be considered as an operator acting in $\dot{\mathcal{E}}$. We shall prove below that $\dot{H}$ is its closure in $\dot{\mathcal{E}}$.

Proposition 5.8. (1) $\rho(\dot{H})=\rho(h, k)$.

(2) In particular, if $\rho(h, k) \neq \varnothing$ then $\dot{H}$ is a closed densely defined operator in $\dot{\mathcal{E}}$ and its spectrum if invariant under complex conjugation.

(3) For $z \in \rho(h, k), z \neq 0$ we have

$$
(\dot{H}-z)^{-1}=\left(\begin{array}{cc}
z^{-1} p(z)^{-1} h-z^{-1} & p(z)^{-1} \\
p(z)^{-1} h & z p(z)^{-1}
\end{array}\right) .
$$


Remark 5.9. It would be tempting to take the expression in (5.4) for $(\dot{H}-z)^{-1}$. The trouble is that $k f_{0}$ does not have an obvious meaning under our assumptions on $k$ if $f_{0} \in|h|^{-\frac{1}{2}} \mathcal{H}$. We obtain a meaningful formula for $(\dot{H}-z)^{-1}$ by noting that $(2 k-z)=z^{-1}(p(z)-h)$ for $z \neq 0$.

Proof. Let us first prove that $\rho(\dot{H}) \subset \rho(h, k)$. Let $z \in \rho(\dot{H})$. Assume first that $z \neq 0$. Then for $g_{1} \in \mathcal{H}$ and $g=\left(0, g_{1}\right) \in \dot{\mathcal{E}}$ there exists a unique

$$
f=\left(f_{0}, f_{1}\right) \in \dot{\mathcal{D}}
$$

such that

$$
(\dot{H}-z) f=g
$$

i.e.

$$
f_{1}=z f_{0} \quad \text { and } \quad p(z) f_{0}=g_{1} .
$$

Since $f_{1}=z f_{0} \in \mathcal{H}$ and $z \neq 0$ it follows that $f_{0} \in\langle h\rangle^{-1} \mathcal{H}$ hence

$$
p(z):\langle h\rangle^{-1} \mathcal{H} \stackrel{\sim}{\longrightarrow} \mathcal{H}
$$

and $z \in \rho(h, k)$.

If $0 \in \rho(\dot{H})$, then for all $\left(g_{0}, g_{1}\right) \in \dot{\varepsilon}$ there exists a unique

$$
\left(f_{0}, f_{1}\right) \in|h|^{-1} \mathcal{H} \cap|h|^{-\frac{1}{2}} \mathcal{H} \oplus\langle h\rangle^{-\frac{1}{2}} \mathcal{H}
$$

with

$$
f_{1}=g_{0} \quad \text { and } \quad h f_{0}+2 k f_{1}=g_{1} .
$$

This implies that

$$
|h|^{-\frac{1}{2}} \mathcal{H}=\langle h\rangle^{-\frac{1}{2}} \mathcal{H},
$$

hence $0 \in \rho(h)$, hence $0 \in \rho(h, k)$.

We now prove that $\rho(h, k) \subset \rho(\dot{H})$ and that (5.10) holds for $z \in \rho(h, k), z \neq 0$.

First, let $z \in \rho(h, k)$ with $z \neq 0, g=\left(g_{0}, g_{1}\right) \in \dot{\mathcal{E}}$, and $\left(f_{0}, f_{1}\right)$ given by the right hand side of (5.10) applied to $g$. We begin by proving that $f \in \dot{\mathcal{D}}$.

Note that

$$
p(z)^{-1} g_{1} \in\langle h\rangle^{-1} \mathcal{H} \subset|h|^{-1} \mathcal{H} \cap|h|^{-\frac{1}{2}} \mathcal{H},
$$

and

$$
h g_{0} \in|h|^{\frac{1}{2}} \mathcal{H} \subset\langle h\rangle^{\frac{1}{2}} \mathcal{H},
$$

hence

$$
p(z)^{-1} h g_{0} \in\langle h\rangle^{-\frac{1}{2}} \mathcal{H} .
$$


It follows that

$$
f_{1}=p(z)^{-1} h g_{0}+z p(z)^{-1} g_{1} \in\langle h\rangle^{-\frac{1}{2}} \mathcal{H} .
$$

The same argument shows that

$$
f_{0}=z^{-1} p(z)^{-1} h g_{0}-z^{-1} g_{0}+p(z)^{-1} g_{1} \in|h|^{-\frac{1}{2}} \mathcal{H} .
$$

It remains to prove that $f_{0} \in|h|^{-1} \mathcal{H}$ i.e. that $h f_{0} \in \mathcal{H}$. Since $p(z)^{-1} g_{1} \in\langle h\rangle^{-1} \mathcal{H}$ it suffices to prove that $z^{-1} h\left(p(z)^{-1} h-1\right) g_{0} \in \mathcal{H}$. Note that

$$
z^{-1} h\left(p(z)^{-1} h-1\right) g_{0}=z^{-1}\left(h p(z)^{-1}-\mathbb{1}\right) h g_{0}=(z-2 k) p(z)^{-1} h g_{0} .
$$

Since $g_{0} \in|h|^{-\frac{1}{2}} \mathcal{H}, h g_{0} \in\langle\dot{h}\rangle^{\frac{1}{2}} \mathcal{H} \subset\langle h\rangle^{\frac{1}{2}} \mathcal{H}$, we obtain that $p(z)^{-1} h g_{0} \in\langle h\rangle^{-\frac{1}{2}} \mathcal{H}$ hence $(z-2 k) p(z)^{-1} h g_{0} \in \mathcal{H}$. This completes the proof of the fact that $f \in \dot{\mathcal{D}}$.

It remains to prove that $(\dot{H}-z) f=g$, which is a standard computation.

Finally assume that $0 \in \rho(h, k)$. Then $0 \in \rho(h)$ which implies that $\dot{\varepsilon}=\mathcal{E}$ and $\dot{H}=H$. Then by Proposition 5.3, $0 \in \rho(H)$. This completes the proof of (1), (3) and of the first statement of (2).

Theorem 5.10. Assume that there exists $z \in \rho(h, k)$ with $z \neq 0$.

(1) Then $\dot{H}$ is self-adjoint on $\left(\dot{\mathcal{E}},\langle\cdot \mid \cdot\rangle_{\mathcal{E}}\right)$ and $\rho(H) \neq \emptyset$.

(2) If in addition $\operatorname{Tr} \mathbb{1}_{-\infty, 0]}(h)<\infty$, then $\dot{H}$ is even-definitizable.

Proof. An easy computation using (5.10) shows that $\left((\dot{H}-z)^{-1}\right)^{\dagger}=(\dot{H}-\bar{z})^{-1}$. Then (1) follows from Lemma 3.3. (2) follows as before from Lemma 5.6.

We now describe the relationship between the two operators $H$ and $\dot{H}$.

Proposition 5.11. (1) $\dot{H}$ is the closure of $H$ in $\dot{\varepsilon}$.

(2) for $z \in \rho(h, k), z \neq 0,(\dot{H}-z)^{-1}$ maps $\mathcal{E}$ into $\mathcal{D}$ and

$$
(H-z)^{-1}=(\dot{H}-z)_{\mid \mathcal{E}}^{-1} .
$$

(2) there exists $C>0$ such that for all $z \in \rho(h, k), z \neq 0$ one has

$$
\left\|(H-z)^{-1} g\right\|_{\mathcal{E}} \leq C\left(\left(1+|z|^{-1}\right)\left\|(\dot{H}-z)^{-1} g\right\|_{\dot{\varepsilon}}+|z|^{-1}\|g\|_{\mathcal{E}}\right), \quad g \in \mathcal{E} .
$$

Proof. If $f=\left(f_{0}, f_{1}\right) \in \dot{\mathcal{D}}$, we pick a sequence $f_{0}^{n} \in\langle h\rangle^{-1} \mathcal{H}$ with $f_{0}^{n} \rightarrow f_{0}$ in $|h|^{-1} \mathcal{H} \cap|h|^{-\frac{1}{2}} \mathcal{H}$. Then $f^{n}=\left(f_{0}^{n}, f_{1}\right) \in \mathcal{D}$ and $f^{n} \rightarrow f$ in $\dot{\mathcal{D}}, H f^{n} \rightarrow \dot{H} f$ in $\dot{\mathcal{E}}$, which proves (1). To prove (2) it suffices to note that $(2 k-z)=z^{-1}(p(z)-h)$ on $\langle h\rangle^{-\frac{1}{2}} \mathcal{H}$, which proves that on $\mathcal{E}$ the right hand side of (5.4) and (5.10) coincide. 
To prove (3) we use that $\|f\|_{\mathcal{E}} \sim\|f\|_{\dot{\varepsilon}}+\left\|f_{0}\right\|_{\mathcal{H}}$. If $f=(H-z)^{-1} g$ then $f_{0}=z^{-1}\left(f_{1}-g_{0}\right)$, hence

$$
\left\|f_{0}\right\|_{\mathcal{H}} \leq|z|^{-1}\left(\left\|f_{1}\right\|_{\mathcal{H}}+\left\|g_{0}\right\|_{\mathcal{H}}\right) \leq|z|^{-1}\left(\left\|(H-z)^{-1} g\right\|_{\dot{\varepsilon}}+\|g\|_{\mathcal{E}}\right),
$$

which proves (3).

Proposition 5.11 has some direct consequences for the estimates on the quadratic pencil that we collect below.

Corollary 5.12. Assume that there exists $z \in \rho(h, k), z \neq 0$ and that

$$
\operatorname{Tr} \mathbb{1}_{]-\infty, 0]}(h)<\infty .
$$

Then we have the estimates on the quadratic pencil

$$
\left\|\langle h\rangle^{\frac{1}{2}} p(z)^{-1}\right\|_{B(\mathcal{H})} \leq\left\{\begin{array}{ll}
C\left(\left(1+|z|^{-1}\right)|\operatorname{Im} z|^{-m-1}+|z|^{-1}\right), & z \in U_{0}(R, a), \\
C\left(\left(1+|z|^{-1}\right)\langle z\rangle^{k}|\operatorname{Im} z|^{-1}+|z|^{-1}\right), & z \in U_{\infty}(R, a) .
\end{array},\right.
$$

and

$$
\left\|p(z)^{-1}\right\|_{B(\mathcal{H})} \leq \begin{cases}\frac{C}{|z|}\left(\left(1+|z|^{-1}\right)|\operatorname{Im} z|^{-m-1}+|z|^{-1}\right), & z \in U_{0}(R, a), \\ \frac{C}{|z|}\left(\left(1+|z|^{-1}\right)\langle z\rangle^{k}|\operatorname{Im} z|^{-1}+|z|^{-1}\right), & z \in U_{\infty}(R, a) .\end{cases}
$$

Proof. By Corollary 3.13 and Proposition 5.11 we obtain

$$
\left\|(H-z)^{-1}\right\|_{B(\varepsilon)} \leq \begin{cases}C\left(\left(1+|z|^{-1}\right)|\operatorname{Im} z|^{-m-1}+|z|^{-1}\right), & z \in U_{0}(R, a), \\ C\left(\left(1+|z|^{-1}\right)\langle z\rangle^{k}|\operatorname{Im} z|^{-1}+|z|^{-1},\right. & z \in U_{\infty}(R, a) .\end{cases}
$$

Using (5.4) we see that we have

$$
\left\|(H-z)^{-1}(0, f)\right\|_{\mathcal{E}}^{2}=\left\|\langle h\rangle^{\frac{1}{2}} p(z)^{-1} f\right\|_{\mathcal{H}}^{2}+|z|^{2}\left\|p(z)^{-1} f\right\|_{\mathcal{H}}^{2},
$$

which gives the result.

\section{Klein-Gordon operators in charge spaces}

In this section we discuss in a way parallel to Section 5 the properties of the operator $K$ in Subsection 4.3 considered as acting on the non-homogeneous charge space $\mathcal{K}_{\frac{1}{4}}$ introduced in Subsection 4.5.

Note that $K$ acting on the homogeneous charge space $\dot{\mathcal{K}}_{\frac{1}{4}}$ could also be considered, at the price of some technical complications. 
6.1. Non-homogeneous charge spaces. In this subsection, we consider a pair of operators $(h, k)$ satisfying the conditions in Subsection 2.2. Note that by duality and interpolation we see that

$$
k \in B\left(\langle h\rangle^{-\theta} \mathcal{H},\langle h\rangle^{\frac{1}{2}-\theta} \mathcal{H}\right), \quad 0 \leq \theta \leq \frac{1}{2} .
$$

We define the (non-homogeneous) charge spaces of order $\theta$

$$
\mathcal{K}_{\theta}:=\langle h\rangle^{-\theta} \mathcal{H} \oplus\langle h\rangle^{\theta} \mathcal{H}, \quad 0 \leq \theta \leq \frac{1}{2} .
$$

and observe that $\mathcal{E} \subset \mathcal{K}_{\theta} \subset \mathcal{E}^{*}$ continuously and densely. Note also that if

$$
q(f, g):=\left(f_{0} \mid g_{1}\right)_{\mathcal{H}}+\left(f_{1} \mid g_{0}\right)_{\mathcal{H}}
$$

then $\left(\mathcal{K}_{\theta}, q\right)$ are Krein spaces.

As we saw in Subsection 4.5, the middle space

$$
\mathcal{F}:=\mathcal{K}_{\frac{1}{4}},
$$

which equals the complex interpolation space $\left[\mathcal{E}, \mathcal{E}^{*}\right]_{\frac{1}{2}}$ is natural even in the case of free Klein-Gordon equations. We will forget the order $\frac{1}{4}$ and call it the nonhomogeneous charge space.

6.2. Klein-Gordon operators on non-homogeneous charge space. We set

$$
\widehat{K}:=\left(\begin{array}{ll}
k & \mathbb{1} \\
h_{0} & k
\end{array}\right) \in B\left(\mathcal{E}, \mathcal{E}^{*}\right) .
$$

Note that there is a simple relation between $\widehat{K}$ and $\hat{H}$ defined in (5.2): indeed, if

$$
\Phi=\Phi(k)=\left(\begin{array}{ll}
\mathbb{1} & 0 \\
k & \mathbb{1}
\end{array}\right) \text { hence } \Phi\left(\begin{array}{l}
f_{0} \\
f_{1}
\end{array}\right)=\left(\begin{array}{c}
f_{0} \\
k f_{0}+f_{1}
\end{array}\right)
$$

then a straightforward computation using (6.1) gives

Lemma 6.1. The map

$$
\Phi=\Phi(k): \mathcal{E}^{*} \longrightarrow \mathcal{E}^{*}
$$

is an isomorphism with inverse

$$
\Phi^{-1}=\Phi(-k)
$$

The subspaces $\mathcal{E}$ and $\mathcal{F}$ are stable under $\Phi$ and the restrictions of $\Phi$ to these subspaces are bijective. We have

$$
\widehat{H} \Phi=\Phi \widehat{K}
$$


Definition 6.2. The charge Klein-Gordon operator is the operator $K$ induced by $\hat{K}$ in $\mathcal{F}$. Its domain is given by

$$
\text { Dom } K:=\{f \in \mathcal{F}: \hat{K} f \in \mathcal{F}\} .
$$

We have

$$
K=\left(\begin{array}{cc}
k & \mathbb{1} \\
h_{0} & \mathbb{1}
\end{array}\right)
$$

Proposition 6.3. (1) One has $\rho(K)=\rho(h, k)$.

(2) In particular, if $\rho(h, k) \neq \emptyset$ then $K$ is a closed, densely defined operator in $\mathcal{F}$ and its spectrum is invariant under complex conjugation.

(3) If $z \in \rho(h, k)$ then

$$
(K-z)^{-1}=\left(\begin{array}{cc}
-p(z)^{-1}(k-z) & p(z)^{-1} \\
\mathbb{1}+(k-z) p(z)^{-1}(k-z) & -(k-z) p(z)^{-1}
\end{array}\right) .
$$

Proof. It suffices to prove (1) and (3). We will set

$$
l=p(z)^{-1} \quad \text { and } \quad u=k-z
$$

to simplify the notation.

Let $z \in \rho(K)$. If $f_{0} \in\langle h\rangle^{-\frac{1}{2}} \mathcal{H}$ and $f_{1}=-u f_{0} \in \mathcal{H}$ then

$$
h_{0} f_{0}+u f_{1}=\left(h_{0}-u^{2}\right) f_{0}=p(z) f_{0},
$$

hence

$$
\left(K_{\theta}-z\right)\left(f_{0}, f_{1}\right)^{t}=\left(0, p(z) f_{0}\right)^{t} .
$$

Thus if $p(z) f_{0}=0$, then $(K-z)\left(f_{0}, f_{1}\right)^{t}=0$; in particular $\left(f_{0}, f_{1}\right)^{t} \in \operatorname{Dom} K$, and so $f_{0}=0$. Hence

$$
p(z):\langle h\rangle^{-\frac{1}{2}} \mathcal{H} \longrightarrow\langle h\rangle^{\frac{1}{2}} \mathcal{H}
$$

is injective. Now let $b \in \mathcal{H}$. Since $(K-z)$ Dom $K=\mathcal{F}$ and $(0, b)^{t} \in \mathcal{F}$, there are $f_{0} \in\langle h\rangle^{-\frac{1}{2}} \mathcal{H}$ and $f_{1} \in \mathcal{H}$ such that $K_{\theta}\left(f_{0}, f_{1}\right)^{t}=(0, b)^{t}$, hence

$$
u f_{0}+f_{1}=0 \text { and } h_{0} f_{0}+u f_{1}=b,
$$

or

$$
p(z) f_{0}=b .
$$


But

$$
p(z)=h-z^{2}+2 z k
$$

hence

$$
h f_{0}=b+z^{2} f_{0}-2 z k f_{0} \in \mathcal{H},
$$

so

$$
f_{0} \in\langle h\rangle^{-1} \mathcal{H}
$$

This proves that

$$
p(z)\langle h\rangle^{-1} \mathcal{H}=\mathcal{H},
$$

and so

$$
p(z):\langle h\rangle^{-1} \mathcal{H} \stackrel{\sim}{\longrightarrow} \mathcal{H},
$$

and $z \in \rho(h, k)$.

Conversely let $z \in \rho(h, k)$, so that

$$
p(z):\langle h\rangle^{-\frac{1}{2}} \mathcal{H} \stackrel{\sim}{\longrightarrow}\langle h\rangle^{\frac{1}{2}} \mathcal{H} .
$$

Let

$$
G=\left(\begin{array}{cc}
-\ell u & \ell \\
\mathbb{1}+u \ell u & -u \ell
\end{array}\right)
$$

be the right hand side of (6.7). Clearly $G \in B\left(\mathcal{E}, \mathcal{E}^{*}\right)$ and a simple computation gives

$$
(\widehat{K}-z) G=\mathbb{1} \text { on } \mathcal{E}^{*}
$$

and

$$
G(\widehat{K}-z)=\mathbb{1} \quad \text { on } \mathcal{E} .
$$

So $G$ is the inverse of

$$
\widehat{K}-z: \mathcal{E} \longrightarrow \mathcal{E}^{*}
$$

If $a \in\langle h\rangle^{-\frac{1}{2}} \mathcal{H}, b \in\langle h\rangle^{\frac{1}{2}} \mathcal{H}$, and $\left(f_{0}, f_{1}\right)^{t}:=G(a, b)^{t}$ then

$$
u f_{0}+f_{1}=a \text { and } h_{0} f_{0}+u f_{1}=b \text {, }
$$

hence

$$
\left(f_{0}, f_{1}\right)^{t} \in \operatorname{Dom} K \text {. }
$$


Thus $G \mathcal{F} \subset \operatorname{Dom} K$. Reciprocally, if $\left(f_{0}, f_{1}\right)^{t} \in \operatorname{Dom} K$ then

$$
(a, b)^{t}:=(K-z)\left(f_{0}, f_{1}\right)^{t}
$$

belongs to $\mathcal{F}$ by (6.6) and

$$
G(a, b)^{t}=\left(f_{0}, f_{1}\right)^{t}
$$

by the preceding computation. Thus Dom $K \subset G \mathcal{F}$. So

$$
G \mathcal{F}=\operatorname{Dom} K
$$

and

$$
K-z: \operatorname{Dom} K \stackrel{\sim}{\longrightarrow} \mathcal{F}
$$

with inverse given by the restriction of $G$ to $\mathcal{F}$.

We deduce from Proposition 6.3 the following analog of Theorem 5.4.

Theorem 6.4. Assume that $\rho(h, k) \neq \emptyset$. Then $K$ is a self-adjoint operator on the Krein space $(\mathcal{F}, q)$ with $\rho(K) \neq \emptyset$.

Since we saw that $\hat{H}=\Phi \widehat{K} \Phi^{-1}$ and $\Phi$ preserves $\mathcal{F}$, it is instructive to describe the operator $\Phi K \Phi^{-1}$. Note that if we compute the image of the canonical Krein structure $q$ on $\mathcal{F}$ under $\Phi$ we get

$$
q^{\prime}(f, g):=q\left(\Phi^{-1} f, \Phi^{-1} g\right)=q(f, g)-2\left(f_{0} \mid k g_{0}\right)_{\mathcal{H}} .
$$

Lemma 6.5. (1) $\Phi K \Phi^{-1}$ is equal to the operator induced by $\hat{H}$ on $\mathcal{F}$.

(2) $\Phi K \Phi^{-1}$ is equal to the restriction of $\hat{H}$ to the domain

$$
\Phi \operatorname{Dom} K=\langle h\rangle^{-3 / 4} \mathcal{H} \oplus\langle h\rangle^{-1 / 4} \mathcal{H}=[\operatorname{Dom} H, \mathcal{E}]_{\frac{1}{2}} .
$$

Proof. (1) is obvious, (2) is a routine computation.

Remark 6.6. As in Section 5, let us make some comments on the relationship between our approach and the one of [24], devoted to Klein-Gordon operators on charge spaces. The comparison between the formulation of the abstract conditions done in Remark 5.5 still applies. In [24], the operator $\widehat{K}$ is considered on the $h_{0}$ version of the charge space $\mathcal{K}_{\frac{1}{4}}$, with equivalent results to those of Section 6 if $h_{0}$ and $h$ are both bounded below. However [24] also consider $\widehat{K}$ on $\mathcal{K}_{0}=\mathcal{H} \oplus \mathcal{H}$, which is not considered in this paper, for reasons explained in Subsection 4.5. 
Another difference is that in [24] the operator $\widehat{K}$ on $\mathcal{K}_{0}$ or $\mathcal{K}_{\frac{1}{4}}$ is studied using the theory of definitizable operators on Krein spaces. In our paper the results for $\widehat{K}$ on $\mathcal{K}_{\frac{1}{4}}$, like existence of a Borel functional calculus or resolvent estimates are deduced from those for $\hat{H}$ on energy spaces, either by duality and interpolation arguments, or by reduction to estimates for the quadratic pencil $p(z)$.

\section{Definitizable Klein-Gordon operators on energy spaces}

In this section, we describe some basic properties of a class of definitizable KleinGordon operators on the energy spaces $\mathcal{E}$ and $\dot{\varepsilon}$. We also describe an approximate diagonalization of $\dot{H}$, which will be useful later on.

We will assume

$$
\begin{aligned}
& \operatorname{Ker} h=\{0\} \\
& \operatorname{Tr} \mathbb{1}_{]-\infty, 0]}(h)<\infty, \\
& k|h|^{-\frac{1}{2}} \in B(\mathcal{H}) .
\end{aligned}
$$

Condition (E1) implies

$$
k\langle h\rangle^{-\frac{1}{2}} \in B(\mathcal{H}),
$$

hence the results of Section 5 hold. Moreover (E2) implies that $h$ is bounded below, hence $\rho(h, k) \neq \emptyset$ by Proposition 2.3.

We set

$$
m^{2}:=\inf \sigma(h) \cap \mathbb{R}^{+}, \quad m \geq 0 .
$$

The constant $m$ is called the mass, Klein-Gordon equations will be called massive resp. massless if $m>0$ resp. $m=0$. A more common name for a massless Klein-Gordon equation is of course a wave equation.

Note that (E1) and (E2) imply that $\sigma_{\text {ess }}(h) \subset \mathbb{R}^{+}$. Moreover if both (E2) and (E2) hold and $m>0$ then $0 \notin \sigma(h)$ hence $|h| \sim\langle h\rangle$ hence $\dot{\mathcal{E}}=\mathcal{E}$ and $\dot{H}=H$. By Theorem 5.10, we obtain that if (E1)-(E3) holds then $\dot{\varepsilon}$ equipped with $\langle\cdot \mid \cdot\rangle_{\mathcal{E}}$ if a Pontryagin space and $\dot{H}$ defined in Definition 5.7 is even-definitizable. 
Proposition 7.1. Assume (E1)-(E3) and let $U$ be a compact set with $U \subset \rho(\dot{H})$ if $m>0$ and $U \subset \rho(\dot{H}) \backslash\{0\}$ if $m=0$. Then there exists $C>0$ such that

$$
\left\|(\dot{H}-z)^{-1}\right\|_{B\left(\dot{\varepsilon}^{*}, \dot{\varepsilon}\right)} \leq C+C\left\|(\dot{H}-z)^{-1}\right\|_{B(\dot{\varepsilon})}, \quad z \in U .
$$

Proof. If $m=0$ and $z \in \rho(\dot{H}), z \neq 0$, then $(\dot{H}-z)^{-1}$ is given by the right hand side of (5.4), using that $k \in B\left(|h|^{-\frac{1}{2}} \mathcal{H}, \mathcal{H}\right)$. This also implies that

$$
p(z)^{-1} h=\mathbb{1}+z p(z)^{-1}(z-2 k) .
$$

Then an easy computation shows that $(\dot{H}-z)^{-1} \in B\left(\dot{\mathcal{E}}^{*}, \dot{\mathcal{E}}\right)$. If $m>0$ and $z \in \rho(\dot{H})$ then the same result holds using that $H=\dot{H}$ and $\dot{\mathcal{E}}=\mathcal{E}$.

Let us prove the bound (7.1). We assume $m=0$, the proof for $m>0$ being simpler. We have

$$
\begin{aligned}
\left\|(\dot{H}-z)^{-1}\right\|_{B\left(\dot{\mathcal{E}}^{*}, \dot{\varepsilon}\right)} \leq C\left(\left\||h|^{\frac{1}{2}} p(z)^{-1}(z-2 k)\right\|_{B(\mathcal{H})}+\left\||h|^{\frac{1}{2}} p(z)^{-1} \epsilon\right\|_{B(\mathcal{H})}\right. \\
\left.+\left\|z p(z)^{-1}(z-2 k)\right\|_{B(\mathcal{H})}+p(z)^{-1}|h|^{\frac{1}{2}} \|_{B(\mathcal{K})}\right) \\
\leq C\left\|\langle h\rangle^{\frac{1}{2}} p(z)^{-1}\langle h\rangle^{\frac{1}{2}}\right\|_{B(\mathcal{K})}, \quad z \in U .
\end{aligned}
$$

Next from the expression (5.10) of $(\dot{H}-z)^{-1}$ we obtain that

$$
\left\||h|^{\frac{1}{2}} p(z)^{-1}\right\|_{B(\mathcal{H})}+\left\|p(z)^{-1}\right\|_{B(\mathcal{H})} \leq C\left\|(\dot{H}-z)^{-1}\right\|_{B(\dot{\mathcal{E}})},
$$

hence

$$
\left\|\langle h\rangle^{\frac{1}{2}} p(z)^{-1}\right\|_{B(\mathcal{H})} \leq C\left\|(\dot{H}-z)^{-1}\right\|_{B(\dot{\varepsilon})} .
$$

Taking adjoints and using that $p(z)^{*}=p(\bar{z})$, we also get

$$
\left\|p(z)^{-1}\langle h\rangle^{\frac{1}{2}}\right\|_{B(\mathcal{H})} \leq C\left\|(\dot{H}-\bar{z})^{-1}\right\|_{B(\dot{\mathcal{\varepsilon}})} \leq C^{\prime}\left\|(\dot{H}-z)^{-1}\right\|_{B(\dot{\varepsilon})},
$$

using (3.4). Since $p(z)^{-1} h=\mathbb{1}+p(z)^{-1}(z-2 k)$, we obtain, for $z \in U$,

$$
\left\|p(z)^{-1} h\right\|_{B(\mathcal{H})} \leq C+C\left\|p(z)^{-1}\langle h\rangle^{\frac{1}{2}}\right\|_{B(\mathcal{H})} \leq C+C\left\|(\dot{H}-z)^{-1}\right\|_{B(\dot{\varepsilon})} .
$$

By (7.3) we have the same bound for $\left\|p(z)^{-1}\langle h\rangle\right\|_{B(\mathcal{H})}$ and for $\left\|\langle h\rangle p(z)^{-1}\right\|_{B(\mathcal{H})}$ by taking adjoints. By interpolation we obtain for $z \in U$

$$
\left\|\langle h\rangle^{\frac{1}{2}} p(z)^{-1}\langle h\rangle^{\frac{1}{2}}\right\|_{B(\mathcal{H})} \leq C+C\left\|(\dot{H}-z)^{-1}\right\|_{B(\dot{\varepsilon})}
$$

which using (7.2) completes the proof of (7.1). 
7.1. Functional calculus. We saw that under conditions (E1), (E2), and (E3'), the operator $\dot{H}$ is even-definitizable, hence admits a $\mathrm{C}^{\alpha}$ and a $\Lambda^{\alpha}$ functional calculus, see Subsects 3.3 and 3.4.

In this subsection, we discuss the functional calculus for $H$, in the case $m=0$, which is not completely straightforward, since in this case $\left(\mathcal{E},\langle\cdot \mid \cdot\rangle_{\mathcal{E}}\right)$ is not a Krein space. We set

$$
\alpha_{H}:=\alpha_{\dot{H}}+\mathbb{1}_{\{0\}},
$$

where $\alpha_{\dot{H}}$ is the order function of $\dot{H}$ (see Definition 3.9).

Proposition 7.2. Assume (E1), (E2), and (E3').

(1) There exists a unique continuous $*$-morphism

$$
C^{\alpha_{H}}(\widehat{\mathbb{R}}) \ni \varphi \longmapsto \varphi(H) \in B(\mathcal{E}),
$$

such that if

$$
\varphi(\lambda)=(\lambda-z)^{-1}, \quad \text { for } z \in \rho(H) \backslash \mathbb{R},
$$

then $\varphi(H)=(H-z)^{-1}$.

(2) There exists a unique extension of the above map to a weakly b-continuous map

$$
\Lambda^{\alpha_{H}}(\widehat{\mathbb{R}}) \ni \varphi \longmapsto \varphi(H) \in B(\mathcal{E}),
$$

which is a norm continuous $*$-morphism.

Proof. By Proposition 5.11,

$$
(H-z)^{-1}=(\dot{H}-z)_{\mid \mathcal{E}}^{-1}, \quad \text { for } z \in \rho(\dot{H}), z \neq 0 .
$$

This implies (see Proposition 3.7) that $\mathbb{1}_{\mathrm{pp}}^{\mathbb{C}}(\dot{H})$ maps $\mathcal{E}$ into itself and defines a bounded projection on $\mathcal{E}$, naturally denoted by $\mathbb{1}_{\mathrm{pp}}^{\mathbb{C}}(H)$, which commutes with $H$. Let us set

$$
\mathcal{E}_{1}:=\left(\mathbb{1}-\mathbb{1}_{\mathrm{pp}}^{\mathbb{C}}(H)\right) \mathcal{\varepsilon},
$$

which is a closed subspace of $\mathcal{E}$, invariant under $(H-z)^{-1}$ for $z \in \rho(H)$. Replacing $H$ by $H_{\mid \varepsilon_{1}}$, we see that without loss of generality we can assume that $\varepsilon_{1}=\mathcal{E}$.

Let us set

$$
\alpha=\alpha_{\dot{H}} \quad \text { and } \quad \beta=\alpha_{H}=\alpha+\mathbb{1}_{\{0\}} .
$$

For $\varphi \in C^{\beta}(\widehat{\mathbb{R}})$ with $\varphi(0)=0$ we set

$$
\tilde{\varphi}(x)=x^{-1} \varphi(x) .
$$


Clearly $\tilde{\varphi} \in C^{\alpha}(\widehat{\mathbb{R}})$ and there exists $C>0$ such that

$$
\|\tilde{\varphi}\|_{\alpha} \leq C\|\varphi\|_{\beta}, \quad \text { for all } \varphi \in C^{\beta}(\widehat{\mathbb{R}}) \text { with } \varphi(0)=0 .
$$

We claim that $\varphi(\dot{H})$ is bounded from $\mathcal{E}$ into itself. In fact if $g \in \mathcal{E}$ we have

$$
\begin{aligned}
\|\varphi(\dot{H}) g\|_{\mathcal{E}} & \leq\|\varphi(\dot{H}) g\|_{\dot{\varepsilon}}+\left\|(\varphi(\dot{H}) g)_{0}\right\|_{\mathcal{H}} \\
& =\|\varphi(\dot{H}) g\|_{\dot{\varepsilon}}+\left\|(\dot{H} \tilde{\varphi}(\dot{H}) g)_{1}\right\|_{\mathcal{H}} \\
& =\|\varphi(\dot{H}) g\|_{\dot{\varepsilon}}+\left\|(\tilde{\varphi}(\dot{H}) g)_{1}\right\|_{\mathcal{H}} \\
& \leq\|\varphi(\dot{H}) g\|_{\dot{\varepsilon}}+\|\tilde{\varphi}(\dot{H}) g\|_{\dot{\varepsilon}} .
\end{aligned}
$$

Moreover from the above inequality, Theorem 3.10 applied to $\dot{H}$ and (7.4) we obtain that

$$
\|\varphi(\dot{H})\|_{B(\mathcal{E})} \leq C\left(\|\varphi\|_{\alpha}+\|\tilde{\varphi}\|_{\alpha}\right) \leq C\|\varphi\|_{\beta} .
$$

Now for $\varphi \in C^{\beta}(\widehat{\mathbb{R}})$ arbitrary we set $\psi(x)=\varphi(x)-\varphi(0)$ and

$$
\varphi(H):=\varphi(0) \mathbb{1}_{\mathcal{E}}+\psi(\dot{H})_{\mid \mathcal{E}} .
$$

From the fact that $(H-z)^{-1}=(H-z)_{\mid \mathcal{E}}^{-1}$, we see that $\varphi(H)=(H-z)^{-1}$ if $\varphi(x)=(x-z)^{-1}$. This yields the existence of the $*$-morphism in (1). The uniqueness follows from the density of the space of bounded rational functions in $C^{\beta}(\widehat{\mathbb{R}})$, see [9, Lemma 4.7]. We deduce (2) from (1) by the argument explained in Appendix B.

Remark 7.3. It is easy to construct a similar functional calculus for the operator $K$ considered in Subsection 6.2. In fact if $\varphi$ belongs to one of the algebras in Proposition 7.2, then $\varphi(H)$ is bounded on $\mathcal{E}$ and thus on $\mathcal{E}^{*}$ by duality. Recalling that $\mathcal{F}=\left[\mathcal{E}, \mathcal{E}^{*}\right]_{1 / 2}$ we see by complex interpolation that $\varphi(H)$ defines a bounded operator on $\mathcal{F}$ with similar estimates. We then define

$$
\varphi(K)=\Phi^{-1} \varphi(H) \Phi,
$$

which is well defined because $\Phi$ and $\Phi^{-1}$ are bounded on $\mathcal{F}$.

7.2. Essential spectrum of Klein-Gordon operators. We now investigate the essential spectrum of the operators $H$ and $\dot{H}$. We set

$$
H_{0}=\left(\begin{array}{ll}
0 & 1 \\
h & 0
\end{array}\right)
$$

defined as in Definition 5.2 for $k=0$, so that Dom $H_{0}=\langle h\rangle^{-1} \mathcal{H} \oplus\langle h\rangle^{-\frac{1}{2}} \mathcal{H}$. 
Similarly we set

$$
\dot{H}_{0}=\left(\begin{array}{ll}
0 & \mathbb{1} \\
h & 0
\end{array}\right),
$$

defined as in Definition 5.7, with domain

$$
\dot{\mathcal{D}}_{0}=|h|^{-1} \mathcal{H} \cap|h|^{-\frac{1}{2}} \mathcal{H} \oplus\langle h\rangle^{-\frac{1}{2}} \mathcal{H} .
$$

Clearly

$$
\sigma_{\text {ess }}\left(H_{0}\right)=\sigma_{\text {ess }}\left(\dot{H}_{0}\right)=\sqrt{\sigma_{\text {ess }}(h)} \cup-\sqrt{\sigma_{\text {ess }}(h)} .
$$

(Recall that from (E1)-(E3) we saw that $\sigma_{\text {ess }}(h) \subset \mathbb{R}^{+}$).

We introduce the condition

$$
k\langle h\rangle^{-\frac{1}{2}} \in B_{\infty}(\mathcal{H}) .
$$

Proposition 7.4. Assume (E1)-(E3) and (A4). Then

$$
(H-z)^{-1}-\left(H_{0}-z\right)^{-1} \in B_{\infty}\left(\mathcal{E}^{*}, \mathcal{E}\right), \quad z \in \rho(H) \cap \rho\left(H_{0}\right),
$$

and

$$
\sigma_{\mathrm{ess}}(H)=\sigma_{\mathrm{ess}}(\dot{H})=\sqrt{\sigma_{\mathrm{ess}}(h)} \cup-\sqrt{\sigma_{\mathrm{ess}}(h)} .
$$

Proof. By (A4) we obtain that $H-H_{0} \in B_{\infty}\left(\mathcal{E}, \mathcal{E}^{*}\right)$ which by the resolvent formula implies that

$$
(H-z)^{-1}-\left(H_{0}-z\right)^{-1} \in B_{\infty}\left(\mathcal{E}^{*}, \mathcal{E}\right) \subset B_{\infty}(\mathcal{E}) .
$$

This implies that

$$
\sigma_{\mathrm{ess}}(H)=\sigma_{\mathrm{ess}}\left(H_{0}\right) \text {. }
$$

Since by $(\mathrm{E} 2) h \mathbb{1}_{\mathbb{R}^{-}}(h) \in B_{\infty}(\mathcal{H})$ we see by the same argument that

$$
\sigma_{\mathrm{ess}}\left(H_{0}\right)=\sigma_{\mathrm{ess}}\left(H_{1}\right)
$$

for

$$
H_{1}=\left(\begin{array}{cc}
0 & \mathbb{1} \\
h \mathbb{1}_{\mathbb{R}^{+}}(h) & 0
\end{array}\right) .
$$

Using the arguments at the beginning of Subsection 7.3, we obtain that

$$
\sigma_{\text {ess }}\left(H_{1}\right)=\sqrt{\sigma_{\text {ess }}(h)} \cup-\sqrt{\sigma_{\text {ess }}(h)},
$$

which proves (2) for $H$.

To prove (2) for $\dot{H}$ we use again the second resolvent formula, hypothesis (A4) and the fact that $\left(\dot{H}_{0}-z\right)^{-1}$ maps $\dot{\mathcal{E}}$ into Dom $\dot{H}_{0}$. We obtain that

$$
\sigma_{\mathrm{ess}}(\dot{H})=\sigma_{\mathrm{ess}}\left(\dot{H}_{0}\right) \text {. }
$$

We conclude as in the case of $H$. 
For completeness we state the following proposition.

Proposition 7.5. Assume (E1)-(E3). Then

$$
\sigma(H)=\sigma(\dot{H}),
$$

and

$$
\sigma_{\mathrm{p}}(H)=\sigma_{\mathrm{p}}(\dot{H}) .
$$

Proof. By Proposition 5.3 (1) and Proposition 5.8, we see that

$$
\rho(H)=\rho(\dot{H})=\rho(h, k),
$$

which proves (1). To prove (2) note that since $H \subset \dot{H}$ we have $\sigma_{\mathrm{p}}(H) \subset \sigma_{\mathrm{p}}(\dot{H})$. Moreover we easily see that

$$
0 \in \sigma_{\mathrm{p}}(H) \Longleftrightarrow 0 \in \sigma_{\mathrm{p}}(h) \Longleftrightarrow 0 \in \sigma_{\mathrm{p}}(\dot{H}) .
$$

Since by (E1) $\operatorname{Ker} h=\{0\}$ we obtain that $0 \notin \sigma_{\mathrm{p}}(H) \cup \sigma_{\mathrm{p}}(\dot{H})$. Finally, in the case $f \in \operatorname{Ker}(\dot{H}-z)$, for $z \neq 0$, we see that $f \in \mathcal{E}$, hence $\dot{H} f=\hat{H} f \in \mathcal{E}$ and $f \in \operatorname{Ker}(H-z)$. Hence $\sigma_{\mathrm{p}}(\dot{H}) \subset \sigma_{\mathrm{p}}(H)$, which completes the proof of (2).

7.3. Approximate diagonalization. It will be convenient later to diagonalize as much as possible the operator $\dot{H}$. This can be done by extracting a convenient positive part from $h$.

We assume that

$$
h=b^{2}-r
$$

with

$$
b \geq 0, \text { self-adjoint on } \mathcal{H}, b^{2} \sim|h|,
$$

and

$$
r \text { symmetric on }\langle h\rangle^{-\frac{1}{2}} \mathcal{H}, b^{-1} r b^{-1} \in B(\mathcal{H}) .
$$

From (A1), (E1), and the Kato-Heinz inequality (see page 123) we see that

$$
\operatorname{Ker} b=\{0\}, \quad\langle b\rangle^{s} \mathcal{H}=\langle h\rangle^{s / 2} \mathcal{H}, \quad b^{s} \mathcal{H}=|h|^{s / 2} \mathcal{H}, \quad|s| \leq 1 .
$$

Set

$$
U:=\frac{1}{\sqrt{2}}\left(\begin{array}{cc}
b & \mathbb{1} \\
b & -1
\end{array}\right) \quad \text { and } \quad U^{-1}=\frac{1}{\sqrt{2}}\left(\begin{array}{cc}
b^{-1} & b^{-1} \\
\mathbb{1} & -1
\end{array}\right)
$$


We see using (7.5) that

$$
U: \dot{\mathcal{E}} \stackrel{\sim}{\longrightarrow} \mathcal{H} \oplus \mathcal{H}=: \mathcal{K}, \quad U: \dot{\mathcal{E}}^{*} \stackrel{\sim}{\longrightarrow} b \mathcal{H} \oplus b \mathcal{H}=\left|L_{0}\right| \mathcal{K},
$$

for

$$
L_{0}:=\left(\begin{array}{cc}
b & 0 \\
0 & -b
\end{array}\right)=U\left(\begin{array}{cc}
0 & \mathbb{1} \\
b^{2} & 0
\end{array}\right) U^{-1} .
$$

We will also use the space

$$
\left\langle L_{0}\right\rangle \mathcal{K}=\langle b\rangle \mathcal{H} \oplus\langle b\rangle \mathcal{H}=\langle h\rangle^{\frac{1}{2}} \mathcal{H} \oplus\langle h\rangle^{\frac{1}{2}} \mathcal{H} .
$$

Note that $\left\langle L_{0}\right\rangle \mathcal{K}=U \mathcal{E}^{*}$ if and only if $m>0$.

We have

$$
\begin{gathered}
L:=U \dot{H} U^{-1}=L_{0}+V_{1}+V_{2}, \\
V_{1}:=k\left(\begin{array}{rr}
\mathbb{1} & -1 \\
-\mathbb{1} & \mathbb{1}
\end{array}\right), \\
V_{2}:=\frac{1}{2} r b^{-1}\left(\begin{array}{rr}
-1 & -1 \\
1 & \mathbb{1}
\end{array}\right) .
\end{gathered}
$$

The canonical Hilbertian scalar product on $\mathcal{K}=\mathcal{H} \oplus \mathcal{H}$ will be denoted by $\langle\cdot \mid \cdot\rangle_{0}$. Then the Krein structure $\langle\cdot \mid \cdot\rangle_{\varepsilon}$ is mapped by $U$ on

$$
\langle u \mid u\rangle=\langle u \mid(\mathbb{1}+K) u\rangle_{0}, \quad K:=-\frac{1}{2} b^{-1} r b^{-1}\left(\begin{array}{ll}
\mathbb{1} & \mathbb{1} \\
\mathbb{1} & \mathbb{1}
\end{array}\right) .
$$

Clearly if both (E1)-(E3) and (A1), as well as (A2) hold then $L$ is even-definitizable on the Krein space $(\mathcal{K},\langle\cdot \mid \cdot\rangle)$.

Lemma 7.6. Assume (E1)-(E3), (A1), and (A2). Then

(1) $L-z: \mathcal{K} \stackrel{\sim}{\rightarrow}\left\langle L_{0}\right\rangle \mathcal{K}$, for $z \in \rho(L)=\rho(\dot{H})$.

(2) Let $U$ be a compact set with $U \subset \rho(\dot{H})$ if $m>0$ and $U \subset \rho(\dot{H}) \backslash\{0\}$ if $m=0$. Then there exists $C>0$ such that

$$
\left\|(L-z)^{-1}\right\|_{B\left(\left\langle L_{0}\right\rangle \mathcal{K}, \mathcal{K}\right)} \leq C 1+C\left\|(L-z)^{-1}\right\|_{B(\mathcal{K})}, \quad \text { for all } z \in U .
$$

Note that Lemma 7.6 would be immediate if $\operatorname{Dom} L=\operatorname{Dom} L_{0}$.

Proof. If $m>0$ we know that $\langle h\rangle \sim|h|$ hence $b^{2} \sim\langle b\rangle^{2}$ by (A1). This implies that $\left\langle L_{0}\right\rangle \mathcal{K}=\left|L_{0}\right| \mathcal{K}$ and the lemma follows from Proposition 7.1 and (7.6). Assume that $m=0$. Then from (A2) we see that $L-z \in B\left(\mathcal{K},\left\langle L_{0}\right\rangle \mathcal{K}\right)$. We note then that

$$
\|u\|_{\langle b\rangle \mathcal{H}} \sim\left\|\mathbb{1}_{[0,1]}(b) u\right\|_{\mathcal{H}}+\left\|\mathbb{1}_{] 1,+\infty[}(b) u\right\|_{b \mathcal{H}}, u \in\langle b\rangle \mathcal{H} .
$$


Proposition 7.1 gives $(\dot{H}-z)^{-1}: \dot{\mathcal{E}}^{*} \rightarrow \dot{\mathcal{E}}$, hence $(L-z)^{-1}:\left|L_{0}\right| \mathcal{K} \rightarrow \mathcal{K}$ by using again (7.6). Since $(L-z)^{-1}: \mathcal{K} \rightarrow \mathcal{K}$, we get from (7.9) $(L-z)^{-1} \in B\left(\left\langle L_{0}\right\rangle \mathcal{K}, \mathcal{K}\right)$ and

$$
\left\|(L-z)^{-1}\right\|_{B\left(\left\langle L_{0}\right\rangle \mathcal{K}, \mathcal{K}\right)} \leq C\left\|(L-z)^{-1}\right\|_{B\left(\left|L_{0}\right| \mathcal{K}, \mathcal{K}\right)}+C\left\|(L-z)^{-1}\right\|_{B(\mathcal{K})} .
$$

Then we apply Proposition 7.1.

We now introduce the condition

$$
k\langle b\rangle^{-1}, \quad b^{-1} r b^{-1} \in B_{\infty}(\mathcal{H}) .
$$

Proposition 7.7. Assume (E1)-(E3) and (A1)-(A3). Then

$$
(L-z)^{-1}-\left(L_{0}-z\right)^{-1} \in B_{\infty}\left(\left\langle L_{0}\right\rangle \mathcal{K}, \mathcal{K}\right), \quad z \in \rho(L) \cap \rho\left(L_{0}\right),
$$

and

$$
\sigma_{\mathrm{ess}}(L)=\sqrt{\sigma_{\mathrm{ess}}(h)} \cup-\sqrt{\sigma_{\mathrm{ess}}(h)} .
$$

Remark 7.8. Note that Proposition 7.7 still holds if we replace $b^{-1} r b^{-1} \in B_{\infty}(\mathcal{H})$ by the weaker condition $\langle b\rangle^{-1} r b^{-1} \in B_{\infty}(\mathcal{H})$. If $b^{-1} r b^{-1} \in B_{\infty}(\mathcal{H})$ then $K$ defined in (7.8) belongs to $B_{\infty}(\mathcal{K})$, which will be useful in Section 8 .

Proof. To prove (1) we use that $(L-z)^{-1},\left(L_{0}-z\right)^{-1} \in B\left(\left\langle L_{0} \mathcal{K}, \mathcal{K}\right)\right.$ by Lemma 7.6, that $V_{1}, V_{2}$ defined in (7.7) belong to $B_{\infty}\left(\mathcal{K},\left\langle L_{0}\right\rangle \mathcal{K}\right)$ and the second resolvent formula. Relation (2) follows from the analogous statement for $\dot{H}$ in Proposition 7.4, noting that (A3) implies (A4).

We will need later the following lemma.

Lemma 7.9. Assume (E1)-(E3) and (A1)-(A3). Let $\chi \in C_{0}^{\infty}(\mathbb{R})$ with $0 \notin \operatorname{supp} \chi$ if $m=0$. Then

$$
\chi(L) \in B\left(\left\langle L_{0}\right\rangle \mathcal{K}, \mathcal{K}\right)
$$

and

$$
\chi(L)-\chi\left(L_{0}\right) \in B_{\infty}(\mathcal{K}) .
$$

Proof. We use the functional calculus formula

$$
\chi(L)=\frac{\mathrm{i}}{2 \pi} \int_{\mathbb{C}} \frac{\partial \tilde{\chi}}{\partial \bar{z}}(z)(z-L)^{-1} d z \wedge d \bar{z} .
$$

Then (1) follows from Lemma 7.6 and the bound in Corollary 3.13. Statement (2) follows from Proposition 7.7 (1) and the fact that the integrals defining $\chi(L)$ and $\chi\left(L_{0}\right)$ are norm convergent. 
We conclude this subsection by discussing the situation when $h_{0}=h+k^{2}$ is positive and by formulating conditions on $k$ which imply conditions (A1)-(A4).

Lemma 7.10. Assume that $h_{0} \geq 0, \operatorname{Ker} h_{0}=\{0\}$ and

- $h_{0} \sim|h|$ and

- $k=k_{1}+k_{2}$ where $k_{i}$ are symmetric on $\langle h\rangle^{-\frac{1}{2}} \mathcal{H}$ and $\left\|k_{1}\left|h_{0}\right|^{-\frac{1}{2}}\right\|_{B(\mathcal{H})}<1$, $k_{1}\left\langle h_{0}\right\rangle^{-\frac{1}{2}}, k_{2}\left|h_{0}\right|^{-\frac{1}{2}} \in B_{\infty}(\mathcal{H})$.

Then conditions (A1)-(A4) are satisfied for

$$
b=\left(h_{0}-k_{1}^{2}\right)^{\frac{1}{2}}, \quad r=k^{2}-k_{1}^{2}=k_{2}^{2}+k_{1} k_{2}+k_{2} k_{1} .
$$

Proof. Since $\left\|k_{1}\left|h_{0}\right|^{-\frac{1}{2}}\right\|<1$ we have $b^{2} \sim h_{0} \sim|h|$, hence (A1) holds. We know $k_{i} \in B\left(b^{-1} \mathcal{H}, \mathcal{H}\right)$ hence $k_{i} \in B(\mathcal{H}, b \mathcal{H})$ by duality. This implies $r \in B\left(b^{-1} \mathcal{H}, b \mathcal{H}\right)$, which is (A2). Similarly we obtain that $k\langle b\rangle^{-1}$ and $b^{-1} r b^{-1}$ belong to $B_{\infty}(\mathcal{H})$.

\section{Mourre estimate for Klein-Gordon operators on energy spaces}

This section is devoted to the proof of a Mourre estimate for Klein-Gordon operators on energy spaces. We will use the approximate diagonalization in Subsection 7.3, and consider the operator $L$.

8.1. Scalar conjugate operators. We start with some preparations with scalar operators, i.e. operators acting on $\mathcal{H}$.

Let us fix as in Subsection 7.3 two operators $b, r$ such that both (A1) and (A2) hold. Let $a$ be a self-adjoint operator on $\mathcal{H}$ such that

$$
b^{2} \in C^{2}(a) .
$$

Then

$$
\chi\left(b^{2}\right): \text { Dom } a \longrightarrow \text { Dom } a \text { for } \chi \in C_{0}^{\infty}(\mathbb{R})
$$

and (see e.g. [13, Subsection 2.2.2])

$$
a_{\chi}:=\chi\left(b^{2}\right) a \chi\left(b^{2}\right)
$$

is essentially self-adjoint on Dom $a$. We still denote by $a_{\chi}$ its closure. Then we have $b^{2} \in C^{2}\left(a_{\chi}\right)$ and $\operatorname{ad}_{a_{\chi}}^{\alpha}\left(b^{2}\right) \in B(\mathcal{H})$, for $0 \leq \alpha \leq 2$. 
Lemma 8.1. Assume (M1). Then

(1) $\mathrm{e}^{\mathrm{i} t a_{\chi}}:\langle b\rangle^{s} \mathcal{H} \rightarrow\langle b\rangle^{s} \mathcal{H}$ and defines a $C_{0}$-group on $\langle b\rangle^{s} \mathcal{H}$ for $|s| \leq 2$,

(2) if $m>0$ or $m=0$ and $0 \notin \operatorname{supp} \chi$ then

$$
\begin{aligned}
\operatorname{ad}_{a_{\chi}}^{\alpha}(b) \in B(\mathcal{H}) & \text { if } 0 \leq \alpha \leq 2 \\
& \text { and } b,\langle b\rangle \in C^{2}\left(a_{\chi} ;\langle b\rangle^{-1} \mathcal{H}, \mathcal{H}\right) \cap C^{2}\left(a_{\chi} ; \mathcal{H},\langle b\rangle \mathcal{H}\right) .
\end{aligned}
$$

Proof. (1) Since $\left[b^{2}, a_{\chi}\right] \in B(\mathcal{H})$, it follows from [8, Appendix $]$ that $\mathrm{e}^{\mathrm{i} t a_{\chi}}$ preserves Dom $b^{2}=\langle b\rangle^{-2} \mathcal{H}$, hence also $\langle b\rangle^{s} \mathcal{H}$ for $|s| \leq 2$ by duality and interpolation. By [1, Proposition 3.2.5], $\mathrm{e}^{\mathrm{i} t a_{\chi}}$ defines a $C_{0}$-group on all these spaces.

(2) If $m>0$ or $m=0$ and $0 \notin \operatorname{supp} \chi$ we have

$$
\operatorname{ad}_{a_{\chi}}(b)=\operatorname{ad}_{a_{\chi}}\left(f\left(b^{2}\right)\right),
$$

for some $f \in C_{0}^{\infty}(\mathbb{R})$. Since $b^{2} \in C^{1}(a)$ we get $\operatorname{ad}_{a_{\chi}}\left(f\left(b^{2}\right)\right) \in B(\mathcal{H})$. The same argument shows that

$$
\operatorname{ad}_{a_{\chi}}^{\alpha}(b)=\chi\left(b^{2}\right) M_{\alpha} \chi\left(b^{2}\right), \quad M_{\alpha} \in B(\mathcal{H}), 0 \leq \alpha \leq 2 .
$$

The same hold for $\langle b\rangle$, which implies (2).

Lemma 8.2. Assume (M1) and let $a_{\chi}$ be defined by (8.1) with $0 \notin$ supp $\chi$. Then

$$
\left\langle a_{\chi}\right\rangle^{-\delta}(\langle b\rangle-b)\left\langle a_{\chi}\right\rangle^{\delta} \in B(\mathcal{H}), \quad 0 \leq \delta \leq 1 .
$$

Proof. The proof is given in Subsection A.3.

We now introduce assumptions on $k$ and $r$.

$$
k\langle b\rangle^{-1},\langle b\rangle^{-1} r b^{-1} \in C^{2}\left(a_{\chi} ; \mathcal{H}\right), \quad b^{-1} r b^{-1} \in C^{1}\left(a_{\chi} ; \mathcal{H}\right) .
$$

Note that if both (E1)-(E3) and (A1), as well as (A2) hold, then $k\langle b\rangle^{-1},\langle b\rangle^{-1} r b^{-1}$, and $b^{-1} r b^{-1}$ belong to $B(\mathcal{H})$, so assumption (M2) makes sense.

Lemma 8.3. Assume (E1)-(E3), (A1), (A2), (M1), and (M2). Then

$$
k, r b^{-1} \in C^{2}\left(a_{\chi} ; \mathcal{H},\langle b\rangle \mathcal{H}\right) .
$$

Proof. Since $\langle b\rangle \in C^{2}\left(a_{\chi} ; \mathcal{H},\langle b\rangle \mathcal{H}\right)$ it suffices to show that $\langle b\rangle^{-1} k,\langle b\rangle^{-1} r b^{-1}$ belong to $C^{2}\left(a_{\chi} ; \mathcal{H}\right)$, which follows from (M2) and [1, Proposition 5.1.7]. 
We now discuss conditions on $k$ which imply (M2), if $h_{0}:=h+k^{2} \geq 0$ and $|h| \sim h_{0}$, similar to Lemma 7.10.

Lemma 8.4. Assume the hypotheses of Lemma 7.10 and choose $b=\left(h_{0}-k_{1}^{2}\right)^{\frac{1}{2}}$ so that $r=k_{2}^{2}+k_{1} k_{2}+k_{2} k_{1}$. Assume moreover that $\left[k_{1}, k_{2}\right]=0$, as an identity in $B\left(\langle h\rangle^{-\frac{1}{2}} \mathcal{H},\langle h\rangle^{\frac{1}{2}} \mathcal{H}\right)$. Then if

$$
k_{1}\langle b\rangle^{-1}, k_{2} b^{-1} \in C^{2}\left(a_{\chi} ; \mathcal{H}\right), \quad b^{-1} k_{1} k_{2} b^{-1} \in C^{1}\left(a_{\chi} ; \mathcal{H}\right)
$$

condition (M2) is satisfied.

Proof. Arguing as in the proof of (8.2) we obtain that $b\langle b\rangle^{-1} \in C^{2}\left(a_{\chi} ; \mathcal{H}\right)$. Since $k_{2} b^{-1} \in C^{2}\left(a_{\chi} ; \mathcal{H}\right)$, we obtain that $k_{2}\langle b\rangle^{-1} \in C^{2}\left(a_{\chi} ; \mathcal{H}\right)$, by [1, Propositions 5.1.7, 5.2.3], hence $k\langle b\rangle^{-1} \in C^{2}\left(a_{\chi} ; \mathcal{H}\right)$. Using that $r=k_{2}^{2}+2 k_{1} k_{2}$ and the same argument, we also obtain the remaining conditions in (M2).

8.2. Conjugate operators for Klein-Gordon operators. We introduce some notation. If $c$ is a closed densely defined operator on $\mathcal{H}$, we set

$$
c_{\text {diag }}:=\left(\begin{array}{ll}
c & 0 \\
0 & c
\end{array}\right), \quad \text { acting on } \mathcal{K}=\mathcal{H} \oplus \mathcal{H} .
$$

We will use the approximate diagonalization introduced in Subsection 7.3. Recall that $U \dot{\mathcal{E}}=\mathcal{K}$ and $U \dot{\mathcal{E}}^{*}=\left|L_{0}\right| \mathcal{K}$.

Let now

$$
A=\left(a_{\chi}\right)_{\text {diag }},
$$

which is the generator of $\left(\mathrm{e}^{\mathrm{i} t a_{\chi}}\right)_{\text {diag }}$ on $\mathcal{K}$.

Proposition 8.5. Assume (E1)-(E3), (A1), (A2), (M1), and (M2). Then

(1) $\mathrm{e}^{\mathrm{i} t A}$ is a $C_{0}$-group on $\left\langle L_{0}\right\rangle \mathcal{K}$,

(2) the Krein structure $\langle\cdot \mid \cdot\rangle$ is of class $C^{1}(A)$,

(3) $L, L_{0}$ belong to $C^{2}\left(A ; \mathcal{K},\left\langle L_{0}\right\rangle \mathcal{K}\right)$ hence to $C^{2}(A)$.

We refer to [9, Subsection 5.5] for the terminology in (2) above.

Proof. (1) follows from Lemma 8.1 (1) and (2) from (M2) and identity (7.8), and (3) from (M2) and identity (7.7).

Proposition 8.6. Assume (E1)-(E3), (A1)-(A3), (M1), and (M2). Let $\chi \in C_{0}^{\infty}(\mathbb{R})$ with $0 \notin \operatorname{supp} \chi$ if $m=0$. Then

$$
\chi(L)[L, \mathrm{i} A] \chi(L)-\chi\left(L_{0}\right)\left[L_{0}, \mathrm{i} A\right] \chi\left(L_{0}\right) \in B_{\infty}(\mathcal{K}) .
$$


Proof. From (E1)-(E3) and (A1)-(A3), we see that

$$
L-L_{0} \in B_{\infty}\left(\mathcal{K},\left\langle L_{0}\right\rangle \mathcal{K}\right) .
$$

From Proposition 8.5 we know that

$$
L-L_{0} \in C^{2}\left(A ; \mathcal{K},\left\langle L_{0}\right\rangle \mathcal{K}\right) \subset C_{\mathrm{u}}^{1}\left(A ; \mathcal{K},\left\langle L_{0}\right\rangle \mathcal{K}\right) .
$$

Therefore

$$
\left[L-L_{0}, \mathrm{i} A\right] \in B_{\infty}\left(\mathcal{K},\left\langle L_{0}\right\rangle \mathcal{K}\right)
$$

We write now

$$
\begin{aligned}
& \chi(L)[L, \mathrm{i} A] \chi(L)-\chi\left(L_{0}\right)\left[L_{0}, \mathrm{i} A\right] \chi\left(L_{0}\right) \\
& =\chi(L)\left[L-L_{0}, \mathrm{i} A\right] \chi(L)+\chi(L)\left[L_{0}, \mathrm{i} A\right]\left(\chi(L)-\chi\left(L_{0}\right)\right) \\
& \quad+\left(\chi(L)-\chi\left(L_{0}\right)\right)\left[L_{0}, \mathrm{i} A\right] \chi\left(L_{0}\right) .
\end{aligned}
$$

By (8.4), the fact that $\left[L_{0}, \mathrm{i} A\right] \in B(\mathcal{K})$, and Lemma 7.9 (2), this is compact.

8.3. Mourre estimate. We denote by $\tau\left(b^{2}, a\right)$ the set of thresholds for $\left(b^{2}, a\right)$. If $a$ is fixed from the context, we will often simply write $\tau\left(b^{2}\right)$ for $\tau\left(b^{2}, a\right)$. So if $\lambda \notin \tau\left(b^{2}, a\right)$ there exists an interval $I \subset \mathbb{R}$, with $\lambda \in I$, a constant $c_{0}>0$ and $R \in B_{\infty}(\mathcal{H})$ such that

$$
\mathbb{1}_{I}\left(b^{2}\right)\left[b^{2}, \mathrm{i} a\right] \mathbb{1}_{I}\left(b^{2}\right) \geq c_{0} \mathbb{1}_{I}\left(b^{2}\right)+R .
$$

We set

$$
\tau(b):=\sqrt{\tau\left(b^{2}\right)} .
$$

In the theorem below we use the notation $c(L)$ for the set of critical points of $L$.

Recall that $\langle\cdot \mid \cdot\rangle_{0}$ denotes the Hilbertian scalar product on $\mathcal{K}$. If $A \in B(\mathcal{H})$ then

$$
A \geq_{0} 0, \quad \text { resp. } A \geq 0,
$$

means that $A$ is self-adjoint positive for $\langle\cdot \mid \cdot\rangle_{0}$, resp. $\langle\cdot \mid \cdot\rangle$.

Theorem 8.7. Assume (E1)-(E3), (A1)-(A3), (M1), and (M2). Let $I \subset \mathbb{R}^{ \pm} a$ compact interval such that

$$
I \cap \pm \tau(b)=\emptyset,
$$

$$
I \cap c(L)=\emptyset,
$$

$$
0 \notin I \text {. }
$$


Let $\chi \in C_{0}^{\infty}(\mathbb{R})$ such that $\chi \equiv 1$ on $I^{2}$ and $0 \notin \operatorname{supp} \chi$ if $m=0$, and $A=\left(a_{\chi}\right)_{\text {diag }}$, where $a_{\chi}$ is defined in (8.1). Then

(1) for $f \in C_{0}^{\infty}\left(\stackrel{\circ}{I)}\right.$ there exists $c_{1}>0$ and $R \in B_{\infty}(\mathcal{K})$ such that

$$
\pm \operatorname{Re}(f(L)[L, \mathrm{i} A] f(L)) \geq c_{1} f^{2}(L)+R ;
$$

(2) if $\lambda \in I \backslash \sigma_{p}(L)$ there exists $\delta>0, c_{2}>0$ such that

$$
\pm \operatorname{Re}\left(\mathbb{1}_{[\lambda-\delta, \lambda+\delta]}(L)[L, \mathrm{i} A] \mathbb{1}_{[\lambda-\delta, \lambda+\delta]}(L)\right) \geq c_{2} \mathbb{1}_{[\lambda-\delta, \lambda+\delta]}(L) .
$$

In (1) and (2) we choose the sign \pm if $I \subset \mathbb{R}^{ \pm}$.

Remark 8.8. We assume for simplicity that $0 \notin I$, even if $m>0$. This is not a restriction since by Proposition 7.7 we know that $0 \notin \sigma_{\text {ess }}(L)$ if $m>0$.

Proof. (1) Since, by (i), $I^{2} \cap \tau\left(b^{2}\right)=\emptyset$, there exists $c_{0}>0, R \in B_{\infty}(\mathcal{H})$ such that

$$
\mathbb{1}_{I^{2}}\left(b^{2}\right)\left[b^{2}, \mathrm{i} a\right] \mathbb{1}_{I^{2}}\left(b^{2}\right) \geq c_{0} \mathbb{1}_{I^{2}}\left(b^{2}\right)+R .
$$

By [13, Theorem 2.2.4] this implies if $\chi \in C_{0}^{\infty}(\mathbb{R})$ is such that $\chi \equiv 1$ on $I^{2}$, there exists $c_{1}>0 R_{1} \in B_{\infty}(\mathcal{H})$ such that

$$
\mathbb{1}_{|I|}(b)\left[b, \mathrm{i} a_{\chi}\right] \mathbb{1}_{|I|}(b) \geq c_{1} \mathbb{1}_{|I|}(b)+R_{1} .
$$

This implies that if $I \subset \mathbb{R}^{ \pm}$one has

$$
\pm \mathbb{1}_{I}\left(L_{0}\right)\left[L_{0}, \mathrm{i} A\right] \mathbb{1}_{I}\left(L_{0}\right) \geq_{0} c_{1} \mathbb{1}_{I}\left(L_{0}\right)+R_{2}, \quad R_{2} \in B_{\infty}(\mathcal{K}),
$$

which implies that for $f \in C_{0}^{\infty}(\stackrel{\circ}{I})$ one has

$$
\pm f\left(L_{0}\right)\left[L_{0}, \mathrm{i} A\right] f\left(L_{0}\right) \geq_{0} c_{1} f^{2}\left(L_{0}\right)+R_{3}, \quad R_{3} \in B_{\infty}(\mathcal{K}) .
$$

Let us now set

$$
\begin{aligned}
B & =f(L)[L, \mathrm{i} A] f(L), & C & =f^{2}(L), \\
B_{0} & =f\left(L_{0}\right)\left[L_{0}, \mathrm{i} A\right] f\left(L_{0}\right), & C_{0} & =f^{2}\left(L_{0}\right),
\end{aligned}
$$

and let $K$ be defined in (7.8). By (A3), we know that $K \in B_{\infty}(\mathcal{K})$. By Lemma 7.9, Proposition 8.6 and hypothesis (A2), we know that

$$
B-B_{0}, C-C_{0}, K \in B_{\infty}(\mathcal{K}) .
$$


We have, for $u \in \mathcal{K}$,

$$
\begin{aligned}
\pm \operatorname{Re}\langle u \mid B u\rangle & = \pm \operatorname{Re}\left\langle u \mid B_{0} u\right\rangle+\operatorname{Re}\langle u \mid R u\rangle \\
& = \pm \operatorname{Re}\left\langle u \mid(\mathbb{1}+K) B_{0} u\right\rangle_{0}+\operatorname{Re}\langle u \mid R u\rangle \\
& = \pm \operatorname{Re}\left\langle u \mid B_{0} u\right\rangle_{0}+\operatorname{Re}\langle u \mid R u\rangle \\
& \geq c_{1}\left\langle u \mid C_{0} u\right\rangle_{0}+\operatorname{Re}\langle u \mid R u\rangle_{0}+\operatorname{Re}\langle u \mid R u\rangle \\
& =c_{1}\langle u \mid C u\rangle+\operatorname{Re}\langle u \mid R u\rangle,
\end{aligned}
$$

where $R$ denotes an element of $B_{\infty}(\mathcal{K})$ and we used (8.7) and (7.8).

(2) Assume now that $\lambda \in I \backslash \sigma_{p}(L)$. Since $I$ does not contain critical points of $L$, we know that $\mathbb{1}_{I}(L) \geq 0$ and that the restriction of $\langle\cdot \mid \cdot\rangle$ to $\mathbb{1}_{I}(L) \mathcal{K}$ is a Hilbertian scalar product, equivalent to $\langle\cdot \mid \cdot\rangle_{0}$, and the restriction of $L$ to $\mathbb{1}_{I}(L) \mathcal{K}$ is self-adjoint in the usual sense for this scalar product. Then (2) follows from (1) by the usual argument.

\section{Limiting absorption principle}

In this section we apply the abstract results from [9] to deduce weighted resolvent estimates from the positive commutator estimate proved in the previous section. The following theorem follows directly from [9, Theorem 7.9], whose hypotheses follow from Theorem 8.7 and Proposition 8.5.

Theorem 9.1. Assume (E1)-(E3), (A1)-(A3), (M1), and (M2).

Let $I \subset \mathbb{R}$ a compact interval such that

$$
\begin{gathered}
I \cap \pm \tau(b)=\emptyset, \\
I \cap c(L)=\emptyset, \\
0 \notin I, \\
I \cap \sigma_{\mathrm{p}}(L)=\emptyset .
\end{gathered}
$$

Let $\chi \in C_{0}^{\infty}(\mathbb{R})$ such that $\chi \equiv 1$ on $I^{2}$ and $0 \notin \operatorname{supp} \chi$ if $m=0$, and $A=\left(a_{\chi}\right)_{\text {diag, }}$, where $a_{\chi}$ is defined in (8.1).

Then there exists $\epsilon_{0}>0$ such that for $\delta>\frac{1}{2}$ one has

$$
\sup _{\operatorname{Re} z \in I, 0<|\operatorname{Im} z| \leq \epsilon_{0}}\left\|\langle A\rangle^{-\delta}(L-z)^{-1}\langle A\rangle^{-\delta}\right\|_{B(\mathcal{K})}<\infty .
$$


9.1. Limiting absorption principle in energy space. After conjugation by the operator $U$ defined in Subsection 7.3, we immediately deduce from Theorem 9.1 a corresponding result on $(\dot{H}-z)^{-1}$ acting on the homogeneous Hilbert space $\dot{\varepsilon}$.

Clearly we have $c(L)=c(\dot{H})$ since $\dot{H}, L$ are unitarily equivalent. Although $H$ is not necessarily definitizable on $\left(\mathcal{E},\langle\cdot \mid \cdot\rangle_{\mathcal{E}}\right)$ if $m=0$, we will still set

$$
c(H):=c(\dot{H}) .
$$

The weights appearing on both sides of $(\dot{H}-z)^{-1}$ are not convenient for applications, at least in the massless case, because they contain the relatively singular operators $b$ and $b^{-1}$ (see (9.1) below). In this subsection we consider the resolvent $(H-z)^{-1}$ on $\mathcal{E}$ and prove more useful resolvent estimates, with non singular weights.

It is convenient to formulate these estimates in terms of an additional operator on $\mathcal{H}$ which dominates the conjugate operator $a_{\chi}$. Let us introduce the corresponding abstract hypothesis.

We fix a self-adjoint operator $\langle x\rangle \geq \mathbb{1}$ on $\mathcal{H}$, called a reference weight, such that

$$
\left\{\begin{array}{lll}
\text { (i) } & \left\langle a_{\chi}\right\rangle\langle x\rangle^{-1} \in B(\mathcal{H}), & \text { for all } \chi \in C_{0}^{\infty}(\mathbb{R}), \\
\text { (ii) } & {\left[\langle b\rangle,\langle x\rangle^{-\delta}\right]\langle x\rangle^{\delta} \in B(\mathcal{H}),} & 0 \leq \delta \leq 1 .
\end{array}\right.
$$

In concrete cases (see Section 12) it is very easy to find a reference weight $\langle x\rangle$.

Theorem 9.2. Assume (E1)-(E3), (A1)-(A3), and (M1)-(M3). Let I $\subset \mathbb{R}$ be an interval as in Theorem 9.1. Then there exists $\epsilon_{0}>0$ such that, for $\frac{1}{2}<\delta \leq 1$,

$$
\sup _{\operatorname{Re} z \in I, 0<|\operatorname{Im} z| \leq \epsilon_{0}}\left\|\left(\langle x\rangle^{-\delta}\right)_{\text {diag }}(H-z)^{-1}\left(\langle x\rangle^{-\delta}\right)_{\text {diag }}\right\|_{B(\varepsilon)}<\infty .
$$

Proof. Set

$$
J=\left\{z \in \mathbb{C}: \operatorname{Re} z \in I, 0<|\operatorname{Im} z| \leq \epsilon_{0}\right\}
$$

Since

$$
U^{-1}\langle A\rangle^{-\delta} U=\left(\begin{array}{cc}
b^{-1}\left\langle a_{\chi}\right\rangle^{-\delta} b & 0 \\
0 & \left\langle a_{\chi}\right\rangle^{-\delta}
\end{array}\right),
$$

we obtain that if $g \in \dot{\varepsilon}$ and $f=(\dot{H}-z)^{-1} g$ one has

$$
\left\|\left\langle a_{\chi}\right\rangle^{-\delta} b f_{0}\right\|_{\mathcal{H}}+\left\|\left\langle a_{\chi}\right\rangle^{-\delta} f_{1}\right\|_{\mathcal{H}} \leq c\left(\left\|\left\langle a_{\chi}\right\rangle^{\delta} b g_{0}\right\|_{\mathcal{H}}+\left\|\left\langle a_{\chi}\right\rangle^{\delta} g_{1}\right\|_{\mathcal{H}}\right), \quad z \in J .
$$


If $g \in \mathcal{E}$, then by Proposition 5.11 we know that $f \in \mathcal{E}$ and $f=(H-z)^{-1} g$. Moreover since $f_{0}=z^{-1}\left(f_{1}-g_{0}\right)$ and $0 \notin I$ we also obtain that

$$
\left\|\left\langle a_{\chi}\right\rangle^{-\delta} f_{0}\right\|_{\mathcal{H}} \leq c\left(\left\|\left\langle a_{\chi}\right\rangle^{\delta} b g_{0}\right\|_{\mathcal{H}}+\left\|\left\langle a_{\chi}\right\rangle^{-\delta} g_{0}\right\|_{\mathcal{H}}+\left\|\left\langle a_{\chi}\right\rangle^{\delta} g_{1}\right\|_{\mathcal{H}}\right), \quad z \in J .
$$

Writing $\langle b\rangle=b+(\langle b\rangle-b)$ and using Lemma 8.2, we obtain that

$$
\left\|\left\langle a_{\chi}\right\rangle^{-\delta}\langle b\rangle f_{0}\right\|_{\mathcal{H}} \leq c\left(\left\|\left\langle a_{\chi}\right\rangle^{-\delta} b f_{0}\right\|_{\mathcal{H}}+\left\|\left\langle a_{\chi}\right\rangle^{-\delta} f_{0}\right\|_{\mathcal{H}}\right),
$$

and

$$
\left\|\left\langle a_{\chi}\right\rangle^{\delta} b g_{0}\right\|_{\mathcal{H}} \leq c\left(\left\|\left\langle a_{\chi}\right\rangle^{\delta}\langle b\rangle g_{0}\right\|_{\mathcal{H}}+\left\|\left\langle a_{\chi}\right\rangle^{\delta} g_{0}\right\|_{\mathcal{H}}\right) .
$$

From condition (M3) (i) we obtain by interpolation that $\left\langle a_{\chi}\right\rangle^{\delta}\langle x\rangle^{-\delta}$ is bounded, hence

$$
\begin{aligned}
\left\|\langle x\rangle^{-\delta}\langle b\rangle f_{0}\right\|_{\mathcal{H}} & \leq c\left(\left\|\left\langle a_{\chi}\right\rangle^{-\delta} b f_{0}\right\|_{\mathcal{H}}+\left\|\langle x\rangle^{-\delta} f_{0}\right\|\right), \\
\left\|\left\langle a_{\chi}\right\rangle^{\delta} b g_{0}\right\|_{\mathcal{H}} & \leq c\left(\left\|\langle x\rangle^{\delta}\langle b\rangle g_{0}\right\|_{\mathcal{H}}+\left\|\langle x\rangle^{\delta} g_{0}\right\|_{\mathcal{H}}\right), \\
\left\|\langle x\rangle^{-\delta} f_{i}\right\|_{\mathcal{H}} & \leq c\left\|\left\langle a_{\chi}\right\rangle^{-\delta} f_{i}\right\|_{\mathcal{H}}, i=0,1, \\
\left\|\left\langle a_{\chi}\right\rangle^{\delta} g_{i}\right\|_{\mathcal{H}} & \leq c\left\|\langle x\rangle^{\delta} g_{i}\right\|_{\mathcal{H}}, i=0,1 .
\end{aligned}
$$

Therefore we deduce from (9.2), (9.3) that

$$
\begin{aligned}
& \left\|\langle x\rangle^{-\delta}\langle b\rangle f_{0}\right\|_{\mathcal{H}}+\left\|\langle x\rangle^{-\delta} f_{0}\right\|_{\mathcal{H}}+\left\|\langle x\rangle^{-\delta} f_{1}\right\|_{\mathcal{H}} \\
& \quad \leq c\left(\left\|\langle x\rangle^{\delta}\langle b\rangle g_{0}\right\|_{\mathcal{H}}+\left\|\langle x\rangle^{\delta} g_{0}\right\|_{\mathcal{H}}+\left\|\langle x\rangle^{\delta} g_{1}\right\|_{\mathcal{H}}\right), \quad z \in J .
\end{aligned}
$$

We use now (M3) (ii) which implies that

$$
\begin{aligned}
\left\|\langle b\rangle\langle x\rangle^{-\delta} f_{0}\right\|_{\mathcal{H}} & \leq\left\|\langle x\rangle^{-\delta}\langle b\rangle f_{0}\right\|_{\mathcal{H}}+\left\|\langle x\rangle^{-\delta} f_{0}\right\|_{\mathcal{H}}, \\
\left\|\langle x\rangle^{\delta}\langle b\rangle g_{0}\right\|_{\mathcal{H}} & \leq\left\|\langle b\rangle\langle x\rangle^{\delta} g_{0}\right\|_{\mathcal{H}} .
\end{aligned}
$$

Therefore (9.5) yields

$$
\left\|b\langle x\rangle^{-\delta} f_{0}\right\|_{\mathcal{H}}+\left\|\langle x\rangle^{-\delta} f_{1}\right\|_{\mathcal{H}} \leq c\left(\left\|\langle b\rangle\langle x\rangle^{\delta} g_{0}\right\|_{\mathcal{H}}+\left\|\langle x\rangle^{\delta} g_{1}\right\|_{\mathcal{H}}\right), \quad z \in J .
$$

Since $\langle b\rangle^{2} \simeq\langle\epsilon\rangle^{2}$, this completes the proof of the theorem.

9.2. Weighted estimates for quadratic pencils. In this subsection we consider weighted estimates for $p(z)^{-1}$. It is natural to introduce the following assumption on $k$ and the reference weight $\langle x\rangle$ :

$$
\langle x\rangle^{\delta} k\langle x\rangle^{-\delta}\langle h\rangle^{-\frac{1}{2}} \in B(\mathcal{H}), \quad \text { for }|\delta| \leq 1 .
$$

Note that (M4) follows from (E2) if $k$ and $\langle x\rangle$ commute, which will be the case in the applications in Section 12. 
Proposition 9.3. Assume (E1)-(E3) and (M4) and let $I \subset \mathbb{R}$ a compact interval with $0 \notin I$, and $0<\delta \leq 1$. Then the following condition are equivalent:

$$
\begin{gathered}
\sup _{\operatorname{Re} z \in I, 0<|\operatorname{Im} z| \leq \epsilon_{0}}\left\|\left(\langle x\rangle^{-\delta}\right)_{\operatorname{diag}}(H-z)^{-1}\left(\langle x\rangle^{-\delta}\right)_{\operatorname{diag}}\right\|_{B(\mathcal{E})}<\infty, \\
\sup _{\operatorname{Re} z \in I, 0<|\operatorname{Im} z| \leq \epsilon_{0}}\left\|\langle h\rangle^{\frac{1}{2}}\langle x\rangle^{-\delta} p(z)^{-1}\langle x\rangle^{-\delta}\right\|_{B(\mathcal{H})}<\infty .
\end{gathered}
$$

Proof. The proof is an easy computation, using formula (5.4), the identity

$$
p(z)^{-1} h=\mathbb{1}+p(z)^{-1} z(z-2 k)
$$

and the fact that $\langle x\rangle^{\delta}(z-2 k)\langle x\rangle^{-\delta}\langle h\rangle^{-\frac{1}{2}}$ is bounded, by (M4). The details are left to the reader.

9.3. Limiting absorption principle in charge space. From Proposition 9.3 we easily get from Theorem 9.2 similar resolvent estimates for $(K-z)^{-1}$ on the charge space $\mathcal{F}$.

Theorem 9.4. Assume (E1)-(E3), (A1)-(A3), and (M1)-(M5). Let $I \subset \mathbb{R}$ an interval as in Theorem 9.1. Then there exists $\epsilon_{0}>0$ such that for $\frac{1}{2}<\delta \leq 1$ one has

$$
\sup _{\operatorname{Re} z \in I, 0<|\operatorname{Im} z| \leq \epsilon_{0}}\left\|\left(\langle x\rangle^{-\delta}\right)_{\text {diag }}(K-z)^{-1}\left(\langle x\rangle^{-\delta}\right)_{\text {diag }}\right\|_{B(\mathcal{F})}<\infty .
$$

Proof. We use formula (6.7) to express $(K-z)^{-1}$. We see that the estimate in the theorem holds if and only if

$$
\sup _{\operatorname{Re} z \in I, 0<|\operatorname{Im} z| \leq \epsilon_{0}}\left\|M_{i}(z)\right\|_{B(\mathcal{H})}<\infty, \quad i=1, \ldots, 4,
$$

for

$$
\begin{aligned}
& M_{1}(z)=\langle h\rangle^{\frac{1}{4}}\langle x\rangle^{-\delta} p(z)^{-1}(k-z)\langle x\rangle^{-\delta}\langle h\rangle^{-\frac{1}{4}}, \\
& M_{2}(z)=\langle h\rangle^{\frac{1}{4}}\langle x\rangle^{-\delta} p(z)^{-1}\langle x\rangle^{-\delta}\langle h\rangle^{\frac{1}{4}}, \\
& M_{3}(z)=\langle h\rangle^{-\frac{1}{4}}\langle x\rangle^{-\delta}\left(\mathbb{1}+(k-z) p(z)^{-1}(k-z)\right)\langle x\rangle^{-\delta}\langle h\rangle^{-\frac{1}{4}}, \\
& M_{4}(z)=\langle h\rangle^{-\frac{1}{4}}\langle x\rangle^{-\delta}(k-z) p(z)^{-1}\langle x\rangle^{-\delta}\langle h\rangle^{-\frac{1}{4}} .
\end{aligned}
$$


Using (M4), duality and interpolation, we see that $\langle h\rangle^{-\frac{1}{4}}\langle x\rangle^{-\delta}(k-z)\langle x\rangle^{\delta}\langle h\rangle^{-\frac{1}{4}}$ is bounded. Therefore the estimate for $M_{2}(z)$ implies the others. By Theorem 9.2 and Proposition 9.3 we know that

$$
\sup _{\operatorname{Re} z \in I, 0<|\operatorname{Im} z| \leq \epsilon_{0}}\left\|\langle h\rangle^{\frac{1}{2}}\langle x\rangle^{-\delta} p(z)^{-1}\langle x\rangle^{-\delta}\right\|_{B(\mathcal{H})}<\infty .
$$

Using duality, the fact that $p(z)^{*}=p(\bar{z})$ and interpolation, this implies the estimate for $M_{2}(z)$. This completes the proof of the theorem.

\section{Existence of the dynamics}

In this section we discuss the existence of the dynamics generated by the operators $H, K$ considered in Sections 5,6, and 7. Note that this not a completely trivial point, since we do not know a priori if these operators are generators of $C_{0}$-groups.

We will assume in this section conditions (E1)-(E3).

10.1. Existence of the dynamics for $\boldsymbol{H}$. If $m>0$ then $H=\dot{H}$ which is evendefinitizable, hence we can define the $C_{0}$-group $\mathrm{e}^{\mathrm{i} t \dot{H}}$, by Subsection 3.5.

If $m=0$ we use now Proposition 7.2 instead of Theorem 3.17. Using the bounded projection $\mathbb{1}_{\mathrm{pp}}^{\mathrm{C}}(H)$ (see the proof of Proposition 7.2), we split $\mathcal{E}$ into the direct sum

$$
\mathcal{E}=\mathcal{E}_{\mathrm{pp}}^{\mathbb{C}}(H) \oplus \mathcal{E}_{1}(H),
$$

both spaces being closed and $H$-invariant, the first one finite-dimensional. We argue as in Subsection 3.5 to construct $\mathrm{e}_{\mid \mathcal{E}_{1}(H)}^{\mathrm{i} t H}$. Thus there exist $C, \lambda>0, n \in \mathbb{N}$ such that

$$
\left\|\left(\mathrm{e}^{\mathrm{i} t H}\right)_{\mid \mathcal{E}_{\mathrm{pp}}^{\mathrm{C}}(H)}\right\| \leq C \mathrm{e}^{\lambda|t|}, \quad\left\|\left(\mathrm{e}^{\mathrm{i} t H}\right)_{\mid \varepsilon_{1}(H)}\right\| \leq C\langle t\rangle^{n}, \quad t \in \mathbb{R} .
$$

10.2. Existence of the dynamics for $\boldsymbol{K}$. We start with a useful observation which is further developed in [9]. Note that the sesquilinear form $q$ defined in (6.3) is defined on $\mathcal{E} \times \mathcal{E}^{*}$ and turns $\left(\mathcal{E}, \mathcal{E}^{*}\right)$ into a dual pair. Since $\Phi$ defined in (6.5) preserves $\mathcal{E}$ and $\mathcal{E}^{*}$ by Lemma 6.3, the same is true of the sesquilinear form $q^{\prime}$ defined in (6.8), (which is equal to $q$ transported by $\Phi$ ).

We check immediately that $\mathrm{e}^{\mathrm{i} t H}$ is unitary for $q^{\prime}$ on $\mathcal{E}$. Therefore by duality $\mathrm{e}^{\mathrm{i} t H}$ extends as a $C_{0}$-group on $\mathcal{E}^{*}$, satisfying (10.1). Since $\mathcal{F}=\left[\mathcal{E}, \mathcal{E}^{*}\right]_{\frac{1}{2}}$, we obtain by interpolation that $\mathrm{e}^{\mathrm{i} t H}$ and hence $\Phi^{-1} \mathrm{e}^{\mathrm{i} t H} \Phi$ extends as $C_{0}$-groups on $\mathcal{F}$. Using also Lemma 6.1 we see that the generator of the later group is $K$, i.e. $\Phi^{-1} \mathrm{e}^{\mathrm{i} t H} \Phi=\mathrm{e}^{\mathrm{i} t K}$. Therefore $\mathrm{e}^{\mathrm{i} t K}$ is a $C_{0}$-group on $\mathcal{F}$, satisfying (10.1). 


\section{Propagation estimates}

In this section we will establish propagation estimates for $\mathrm{e}^{\mathrm{i} t \dot{H}}, \mathrm{e}^{\mathrm{i} t H}$ and $\mathrm{e}^{\mathrm{i} t K}$. We will need the following assumption.

(M5) $D(\langle x\rangle) \cap D\left(b^{2}\right)$ is dense in $D\left(b^{2}\right), \mathrm{e}^{\mathrm{i} s\langle x\rangle}$ sends $D\left(b^{2}\right)$ into itself, and $\left[\langle x\rangle, b^{2}\right]$ extends to a bounded operator from $D\left(b^{2}\right)$ to $\mathcal{H}$ which we denote $\left[\langle x\rangle, b^{2}\right]_{0}$.

Note that (M5) implies (see [1, Proposition 3.2.5])

$$
\sup _{0 \leq s \leq 1}\left\|b^{2} \mathrm{e}^{\mathrm{i} s\langle x\rangle} u\right\|<\infty
$$

for all $u \in D\left(b^{2}\right)$.

We assume (E1)-(E3), (A1)-(A4), (M1)-(M3), and (M5) in the following. We also suppose that $\left[k_{i},\langle x\rangle\right]=0$ for $i=1,2$ which implies in particular (M4).

Lemma 11.1. If $z \in \rho(h, k)$ and $0 \leq \delta \leq 1$ then $p^{-1}(z)$ sends $D\left(\langle x\rangle^{\delta}\right)$ into itself.

Proof. We first show $p^{-1}(z)$ sends $D(\langle x\rangle)$ into itself. Let $u \in D(\langle x\rangle)$. We have to show that

$$
\sup _{|t| \leq 1}\left\|\frac{\mathrm{e}^{\mathrm{i} t\langle x\rangle}-1}{t} p^{-1}(z) u\right\|<\infty .
$$

We write

$$
\begin{aligned}
\frac{\mathrm{e}^{\mathrm{i} t\langle x\rangle}-1}{t} p^{-1}(z) u= & p^{-1}(z) \frac{\mathrm{e}^{\mathrm{i} t\langle x\rangle}-1}{t} u \\
& +\mathrm{e}^{\mathrm{i} t\langle x\rangle} \frac{p^{-1}(z)-\mathrm{e}^{-i t\langle x\rangle} p^{-1}(z) \mathrm{e}^{\mathrm{i} t\langle x\rangle}}{t} u .
\end{aligned}
$$

Clearly

$$
\sup _{|t| \leq 1}\left\|p^{-1}(z) \frac{\mathrm{e}^{\mathrm{i} t\langle x\rangle}-1}{t} u\right\|<\infty .
$$

Let us now consider the second term. We have

$$
\begin{aligned}
& \frac{p^{-1}(z)-\mathrm{e}^{-\mathrm{i} t\langle x\rangle} p^{-1}(z) \mathrm{e}^{\mathrm{i} t\langle x\rangle}}{t} u \\
& \quad=\mathrm{e}^{-\mathrm{i} t\langle x\rangle} p^{-1}(z) \mathrm{e}^{\mathrm{i} t\langle x\rangle} \frac{\mathrm{e}^{-\mathrm{i} t\langle x\rangle} b^{2} \mathrm{e}^{\mathrm{i} t\langle x\rangle}-b^{2}}{t} p^{-1}(z) u,
\end{aligned}
$$

where we have used that $\mathrm{e}^{\mathrm{i} s\langle x\rangle}$ sends $D\left(b^{2}\right)$ into itself. Now note that

$$
\frac{\mathrm{e}^{-\mathrm{i} t\langle x\rangle} b^{2} \mathrm{e}^{\mathrm{i} t\langle x\rangle}-b^{2}}{t}=\frac{1}{t} \int_{0}^{t} \mathrm{e}^{-\mathrm{i} \theta\langle x\rangle}\left[\langle x\rangle, b^{2}\right]_{0} \mathrm{e}^{\mathrm{i} \theta\langle x\rangle} d \theta .
$$


It follows using (11.2) that

$$
\sup _{|t| \leq 1}\left\|\mathrm{e}^{-\mathrm{i} t\langle x\rangle} p^{-1}(z) \mathrm{e}^{\mathrm{i} t\langle x\rangle} \frac{\mathrm{e}^{-\mathrm{i} t\langle x\rangle} b^{2} \mathrm{e}^{\mathrm{i} t\langle x\rangle}-b^{2}}{t} p^{-1}(z)\right\|<\infty
$$

and thus the lemma for $\delta=1$. The lemma for $\delta=0$ is obvious, the general case follows by interpolation.

Corollary 11.2. For all $0 \leq \delta \leq 1$ and $\chi \in C_{0}^{\infty}(\mathbb{R})$ the operators $\langle x\rangle^{\delta} \chi(H)\langle x\rangle^{-\delta}$ and $\langle x\rangle^{\delta} \chi(K)\langle x\rangle^{-\delta}$ are bounded.

Proof. By an interpolation argument it is sufficient to consider the case $\delta=1$. Using (5.4) and the fact that $[\langle x\rangle, k]=0$ we see that for $z \in \rho(h, k)$ we have

$$
\langle x\rangle(H-z)^{-1}\langle x\rangle^{-1}=\left[\begin{array}{ll}
0 & 0 \\
1 & 0
\end{array}\right]+\langle x\rangle p^{-1}(z)\langle x\rangle^{-1}\left[\begin{array}{cc}
z-2 k & 1 \\
-z(2 k-z) & z
\end{array}\right] .
$$

By the definition of the smooth functional calculus for $H$ it is sufficient to show that $\langle x\rangle p^{-1}(z)\langle x\rangle^{-1}$, which is bounded on $\mathcal{H}$ by Lemma 11.1, fulfills suitable resolvent estimates. Using Lemma 11.1 we can write the commutator

$$
\left[\langle x\rangle, p^{-1}(z)\right]=p^{-1}(z)\langle h\rangle^{\frac{1}{2}}\langle h\rangle^{-\frac{1}{2}}\left[b^{2},\langle x\rangle\right]_{0} p^{-1}(z) .
$$

It is now sufficient to apply Corollary 5.12 to obtain the required estimates. By duality $\langle x\rangle^{\delta} \chi(H)\langle x\rangle^{-\delta}$ is bounded on $\mathcal{E}^{*}$ and thus on $\mathcal{F}$ by complex interpolation. To obtain the result for $K$ we use that

$$
\chi(K)=\Phi^{-1} \chi(H) \Phi,
$$

that $\Phi$ commutes with $\langle x\rangle$ and that $\Phi, \Phi^{-1}$ are continuous on $\mathcal{F}$.

Proposition 11.3. Let $I \subset \mathbb{R}$ an interval as in Theorem 9.1 and let $\chi \in C_{0}^{\infty}$, and supp $\chi \subset I$. Suppose $\frac{1}{2}<\delta \leq 1$. Then there exists $C>0$ such that

$$
\int_{\mathbb{R}}\left\|\langle x\rangle^{-\delta} \mathrm{e}^{\mathrm{i} t H} \chi(H)\langle x\rangle^{-\delta} f\right\|_{\mathcal{E}}^{2} d t \leq C\|f\|_{\mathcal{E}}^{2},
$$

and

$$
\int_{\mathbb{R}}\left\|\langle x\rangle^{-\delta} \mathrm{e}^{\mathrm{i} t K} \chi(K)\langle x\rangle^{-\delta} f\right\|_{\mathcal{F}}^{2} d t \leq C\|f\|_{\mathcal{F}}^{2}
$$


Proof. We first prove (11.3). Note that by Theorem 9.2 there exists $\epsilon_{0}>0$ such that one has

$$
\sup _{0<|\operatorname{Im} z| \leq \epsilon_{0}}\left\|\left(\langle x\rangle^{-\delta}\right)_{\text {diag }}(H-z)^{-1} \chi(z)\left(\langle x\rangle^{-\delta}\right)_{\text {diag }}\right\|_{B(\varepsilon)}<\infty .
$$

We now have to show that we can replace $\chi(z)$ by $\chi(H)$. We choose $\tilde{\chi} \in C_{0}^{\infty}(I)$ with $\tilde{\chi} \chi=\chi$. We write

$$
\begin{aligned}
&\left\|\langle x\rangle^{-\delta}(H-z)^{-1} \chi(H)\langle x\rangle^{-\delta} f\right\|_{B(\varepsilon)}^{2} \\
& \leq C\left\|\langle x\rangle^{-\delta}(H-z)^{-1} \tilde{\chi}(z) \chi(H)\langle x\rangle^{-\delta} f\right\|_{B(\varepsilon)}^{2} \\
&+C\left\|\langle x\rangle^{-\delta}(H-z)^{-1}(1-\tilde{\chi}(z)) \chi(H)\langle x\rangle^{-\delta} f\right\|_{B(\varepsilon)}^{2} .
\end{aligned}
$$

The estimate for the first term follows from the estimate with $\chi(z)$ and Corollary 11.2. Let us treat the second term. We claim

$$
\left\|\langle x\rangle^{-\delta}(H-(\lambda+\mathrm{i} \epsilon))^{-1}(1-\tilde{\chi}(\lambda)) \chi(H)\langle x\rangle^{-\delta} f\right\|_{B(\varepsilon)}^{2} \leq C\langle\lambda\rangle^{-2},
$$

uniformly in $\epsilon$. Let

$$
f_{\lambda}^{\epsilon}(x)=\langle\lambda\rangle \frac{1}{x-(\lambda+\mathrm{i} \epsilon)}(1-\tilde{\chi}(\lambda)) \chi(x) .
$$

It is sufficient to show that all the semi-norms $\left\|f_{\lambda}^{\epsilon}\right\|_{m}$ are uniformly bounded with respect to $\lambda, \epsilon$. Note that $g_{\lambda}(x)=(1-\tilde{\chi}(\lambda)) \chi(x)$ vanishes to all orders at $x=\lambda$. If supp $\chi \subset[-C, C]$ this is enough to assure that $\left\|f_{\lambda}^{\epsilon}\right\|_{m}$ is uniformly bounded in $\lambda \in[-2 C, 2 C]$ and $\epsilon>0$. For $|\lambda| \geq 2 C$ we observe that

$$
\left|\langle\lambda\rangle \frac{1}{x-(\lambda+\mathrm{i} \epsilon)}\right| \leq C
$$

with analogous estimates for the derivatives. Thus the second term in (11.5) is also uniformly bounded in $0<|\operatorname{Imz}| \leq \epsilon_{0}$.

We now write

$$
\begin{gathered}
\left((H-(\lambda+\mathrm{i} \epsilon))^{-1}-(H-(\lambda-\mathrm{i} \epsilon))^{-1}\right) \chi(H) f \\
=i \int_{-\infty}^{\infty} \mathrm{e}^{-\epsilon|t|} \mathrm{e}^{\mathrm{i} \lambda t} \mathrm{e}^{-\mathrm{i} H t} \chi(H) f d t,
\end{gathered}
$$

the integral being norm convergent by (10.1). By Plancherel's formula this yields

$$
\begin{gathered}
\int_{-\infty}^{\infty} \|\langle x\rangle^{-\delta}\left(\left(H-(\lambda+\mathrm{i} \epsilon)^{-1}-(H-(\lambda-\mathrm{i} \epsilon))^{-1}\right) \chi(H)\langle x\rangle^{-\delta} f \|_{\varepsilon}^{2} d \lambda\right. \\
=\int_{-\infty}^{\infty} \mathrm{e}^{-2 \epsilon|t|}\left\|\langle x\rangle^{-\delta} \mathrm{e}^{-\mathrm{i} t H} \chi(H)\langle x\rangle^{-\delta} f\right\|_{\varepsilon}^{2} d t .
\end{gathered}
$$


The left hand side of this equation is uniformly bounded in $\epsilon$ with $\epsilon$ small enough, which implies (11.3).

Let us now prove (11.4). First note that by duality we can replace $B(\varepsilon)$ by $B\left(\mathcal{E}^{*}\right)$ in (11.3). This gives (11.4) with $K$ replaced by $H$ by complex interpolation. We then use that

$$
\mathrm{e}^{\mathrm{i} t K} \chi(K)=\Phi^{-1} \mathrm{e}^{\mathrm{i} t H} \chi(H) \Phi,
$$

that $\langle x\rangle^{-\delta}$ commutes with $\Phi$ and that $\Phi, \Phi^{-1}$ are bounded operators on $\mathcal{F}$.

\section{Examples}

In this section we describe examples of Klein-Gordon equations to which the abstract results of Sections 7, 8, and 9 can be applied.

Let us first discuss how to check the abstract hypotheses (E1)-(E3). In the case $\inf \sigma_{\text {ess }}(h)>0$, the only delicate condition is (E1). In fact in this case (E1) implies (E2) and also that $0 \notin \sigma(h)$. Therefore $|h| \sim\langle h\rangle$. It follows that if $\epsilon \geq 0$ is a self-adjoint operator such that Dom $h=\operatorname{Dom} \epsilon^{2}$ we have $|h| \sim\langle h\rangle \sim\langle\epsilon\rangle^{2}$ and hence in condition (E3) we can replace $|h|^{-\frac{1}{2}}$ by $\langle\epsilon\rangle^{-1}$.

Similarly if $b$ is an operator such that (A1) holds, we have $b^{2} \sim\langle\epsilon\rangle^{2}$ and in conditions (A2)-(A4) we can replace $b^{-1}$ and $\langle b\rangle^{-1}$ by $\langle\epsilon\rangle^{-1}$.

If inf $\sigma_{\text {ess }}(h)=0$ then both (E1) and (E2) are important. Moreover it is again important to find a self-adjoint operator $\epsilon \geq 0$ such that $|h| \sim \epsilon^{2}$. An abstract result allowing to do this is given in the following proposition.

Proposition 12.1. Let $\epsilon \geq 0$ be a self-adjoint operator on $\mathcal{H}$ and $r_{1}, r_{2}$ two symmetric operators on Dom $\epsilon$ such that

$$
\operatorname{Ker} \epsilon=\{0\},\left\|r_{1} \epsilon^{-1}\right\|<1, \quad r_{2} \epsilon^{-1} \in B_{\infty}(\mathcal{H}) .
$$

If $h=\epsilon^{2}-r_{1}^{2}-r_{2}^{2}$ as an identity in $B\left(\langle\epsilon\rangle^{-1} \mathcal{H},\langle\epsilon\rangle \mathcal{H}\right)$ and $\operatorname{Ker} h=\{0\}$, then

$$
\begin{cases}(1) & \operatorname{Tr} \mathbb{1}_{]-\infty, 0]}(h)<\infty, \\ \text { (2) } & |h| \sim \epsilon^{2} .\end{cases}
$$

The proof will be given in Subsection A.2.

12.1. Charged Klein-Gordon equations on scattering manifolds. Let $\mathcal{N}$ be a smooth, $d-1$ dimensional compact manifold whose elements are denoted by $\omega$. We consider a $d$ dimensional manifold

$$
\left.\mathcal{M} \simeq \mathcal{M}_{0} \cup\right] 1,+\infty[\times \mathcal{N},
$$


where $\mathcal{M}_{0} \Subset \mathcal{M}$ is relatively compact. For $m \in \mathbb{R}$ we denote by $S^{m}(\mathcal{M})$ the space of real valued functions $f \in C^{\infty}(\mathcal{M})$ such that

$$
\left|\partial_{s}^{k} \partial_{\omega}^{\alpha} f(s, \omega)\right| \leq C_{k, \alpha} s^{m-k} \quad \text { for }(s, \omega) \in\left[1, \infty\left[\times \mathcal{N} \text {, for all } k \in \mathbb{N}, \alpha \in \mathbb{N}^{d-1} .\right.\right.
$$

Definition 12.2. A Riemannian metric $g^{0}$ on $\mathcal{M}$ is called conic if there exists $R>0$ and a Riemannian metric $h$ on $\mathcal{N}$ such that

$$
g^{0}=d s^{2}+s^{2} h_{j k}(\omega) d \omega^{j} d \omega^{k}, \quad(s, \omega) \in[R, \infty[\times \mathcal{N} .
$$

A Riemannian metric $g$ on $\mathcal{M}$ is called a scattering metric if

$$
g=g^{0}+\tilde{g},
$$

where $g^{0}$ is a conic metric and $\tilde{g}$ is of the form

$$
\tilde{g}=m^{0}(s, \omega) d s^{2}+s m_{j}^{1}(s, \omega)\left(d s d \omega^{j}+d \omega^{j} d s\right)+s^{2} m_{j k}^{2}(s, \omega) d \omega^{j} d \omega^{k}
$$

with $m^{l} \in S^{-\mu_{l}}(\mathcal{M})$ for $l=0,1,2, \mu_{l}>0$.

We will assume in the sequel that $g$ is a scattering metric on $\mathcal{M}$ in the sense of the above definition. We consider a charged Klein-Gordon field $\phi$ on $\mathcal{M}$ minimally coupled to an external electromagnetic field described by the electric potential $v(s, \omega)$ and the magnetic potential $A_{k}(s, \omega) d x^{k}$. It fulfills the Klein-Gordon equation

$$
\left(\partial_{t}-\mathrm{i} v\right)^{2} \phi-\left(\nabla^{k}-\mathrm{i} A^{k}\right)\left(\nabla_{k}-\mathrm{i} A_{k}\right) \phi+m^{2} \phi=0 .
$$

Here $\nabla$ is the Levi-Civita connection associated to the metric $g$. The function $m(s, \omega)$ on $\mathcal{M}$ corresponds to a variable mass term. The above equation writes in local coordinates

$$
\left(\partial_{t}-\mathrm{i} v\right)^{2} \phi-|g|^{-1 / 2}\left(\partial_{j}-\mathrm{i} A_{j}\right)|g|^{1 / 2} g^{j k}\left(\partial_{k}-\mathrm{i} A_{k}\right) \phi+m^{2}(s, \omega) \phi=0,
$$

where $g^{j k}=\left(g_{j k}\right)^{-1},|g|=\operatorname{det}\left(g_{j k}\right)$. We denote by

$$
d v=|g|^{\frac{1}{2}} d s d \omega
$$

the Riemannian volume element on $(\mathcal{M}, g)$. Putting $\psi=|g|^{1 / 4} \phi$ we see that $\psi$ solves

$$
\begin{aligned}
& \left(\partial_{t}-\mathrm{i} v\right)^{2} \psi-|g|^{-1 / 4}\left(\partial_{j}-\mathrm{i} A_{j}\right)|g|^{1 / 2} g^{j k}\left(\partial_{k}-\mathrm{i} A_{k}\right)|g|^{-1 / 4} \psi \\
& \quad+m^{2}(s, \omega) \psi=0,
\end{aligned}
$$

which is the equation we will consider. 
Remark 12.3. The equation (12.2) can be seen as a Klein-Gordon equation on the lorentzian manifold $\mathbb{R} \times \mathcal{N}$ with metric $d t^{2}-g$. Our results easily generalize to the metric $c(s, \omega) d t^{2}-g$ where $0<c_{1} \leq c(s, \omega) \leq c_{1}^{-1}$ is a smooth function tending to 1 at infinity. The generalization reduces to a simple change of unknown function, see [12, Section 2.1] for details.

We set

$$
h_{0}:=-g^{-1 / 4}\left(\partial_{j}-\mathrm{i} A_{j}\right) g^{1 / 2} g^{j k}\left(\partial_{k}-\mathrm{i} A_{k}\right) g^{-1 / 4}+m^{2}(s, \omega)
$$

acting on $\mathcal{H}=L^{2}(\mathcal{N} ; d s d \omega)$, equipped with its canonical scalar product. Let also

$$
p=-g^{-1 / 4} \partial_{j} g^{1 / 2} g^{j k} \partial_{k} g^{-1 / 4} \text {. }
$$

We assume that

$$
\left\{\begin{array}{l}
A_{j}(s, \omega), m(s, \omega)-m_{\infty} \in S^{-\mu_{0}}(\mathcal{M}) \\
\text { for some } \mu_{0}>0, m_{\infty}:=\lim _{s \rightarrow \infty} m(s, \omega) \geq 0 .
\end{array}\right.
$$

The operator $k$ is assumed to be a multiplication operator $k=v(s, \omega)$ with

$$
\begin{cases}v(s, \omega)=v_{\mathrm{l}}(s, \omega)+v_{\mathrm{s}}(s, \omega), & v_{\mathrm{l}}(s, \omega) \in S^{-\mu_{0}}(\mathcal{M}), \\ v_{\mathrm{s}}(s, \omega)\langle p\rangle^{-1 / 2} \in B_{\infty}(\mathcal{H}), & \langle s\rangle^{2} v_{\mathrm{s}}(s, \omega)\langle p\rangle^{-1 / 2} \in B(\mathcal{H}) .\end{cases}
$$

It follows that $h=h_{0}-k^{2}$ is self-adjoint and bounded below with $\langle h\rangle \sim\left\langle h_{0}\right\rangle$ and $\sigma_{\mathrm{ess}}(h)=\left[m_{\infty}^{2},+\infty[\right.$.

As scalar conjugate operator we choose as usual the generator of dilations

$$
a=\frac{1}{2}\left(\eta(s) s D_{s}+D_{s} s \eta(s)\right),
$$

where $\eta \in C^{\infty}\left(\mathbb{R}, \mathbb{R}^{+}\right)$with $\eta(s)=1$ for $s \geq 2$ and $\eta(s)=0$ for $s \leq 1$. As reference weight we choose

$$
\langle x\rangle=\left(s^{2}+1\right)^{\frac{1}{2}} .
$$

12.1.1. Massive case. We consider massive Klein-Gordon equations i.e. $m=$ $\inf \sigma(h) \cap \mathbb{R}^{+}>0$. This implies that $m_{\infty}>0$.

Proposition 12.4. Assume (12.4), (12.5), $m_{\infty}>0$ and $\mathrm{Ker} h=\{0\}$. Then

(1) conditions (E1)-(E3), (A1)-(A4), and (M1)-(M5) are satisfied;

(2) one has $\tau(b)=\left\{m_{\infty}\right\}$. 
Proof. To check (E1)-(E3) we use Proposition 12.1 with $\epsilon=h_{0}^{\frac{1}{2}}, r_{1}=\mathbb{1}_{\{|x| \geq R\}} v$, $r_{2}=\mathbb{1}_{\{|x| \leq R\}} v$. Clearly $r_{2} \epsilon^{-1} \in B_{\infty}(\mathcal{H})$, and since $\mathrm{s}-\lim _{R \rightarrow \infty} \mathbb{1}_{\{|x| \geq R\}}=0$, we deduce from (12.5) that $\left\|r_{1} \epsilon^{-1}\right\|<1$ for $R$ large enough. Moreover $|h| \sim h_{0} \sim$ $D_{s}^{2}-\frac{1}{s^{2}} \Delta_{\mathcal{N}}+1$.

To check (A1)-(A4) we use Lemma 7.10. We fix smooth cutoff functions $F_{0}$, $F_{\infty} \in C^{\infty}(\mathbb{R})$ with

$$
\operatorname{supp} F_{0} \subset[-2,2], \quad F_{0} \equiv 1 \text { in }[-1,1], F_{0}+F_{\infty}=1 .
$$

We split $k$ as $k_{1}+k_{2}$ with

$$
k_{1}=F_{\infty}\left(R^{-1} s\right) v_{1}, \quad k_{2}=v_{\mathrm{s}}+F_{0}\left(R^{-1} s\right) v_{1} .
$$

Since $F_{0}\left(R^{-1}|x|\right) v_{1}$ satisfies the same conditions as $v_{\mathrm{S}}$ we can assume that

$$
k_{2}=v_{\mathrm{s}}
$$

in the sequel. As before for $R \gg 1$ we have

$$
\left\|k_{1} \epsilon^{-1}\right\|<1, \quad k_{1}\langle\epsilon\rangle^{-1}, k_{2} \epsilon^{-1} \in B_{\infty}(\mathcal{H}) .
$$

By Lemma 7.10 conditions (A1)-(A4) are satisfied for

$$
b=\left(\epsilon^{2}-k_{1}^{2}\right)^{\frac{1}{2}} \text { and } \quad r=k_{2}^{2}+2 k_{1} k_{2} .
$$

Condition (M1) is clearly satisfied. To check (M2) we apply Lemma 8.4 and check (M2') instead. It is a standard fact that $\operatorname{ad}_{a_{\chi}}^{\alpha}\left(k_{1}\langle b\rangle^{-1}\right) \in B(\mathcal{H})$ (one may for example use pseudo-differential calculus on scattering manifolds, see e.g. [26, Chapter 6.3] for an overview of this calculus). Therefore $k_{1}\langle b\rangle^{-1} \in C^{2}\left(a_{\chi} ; \mathcal{H}\right)$. Since $0 \notin \sigma(b)$ we also see that $k_{1} b^{-1} \in C^{2}\left(a_{\chi} ; \mathcal{H}\right)$.

The same type of argument shows that

$$
a_{\chi}\langle s\rangle^{-1}\langle p\rangle^{-1 / 2}, a_{\chi}^{2}\langle s\rangle^{-2}\langle p\rangle^{-1 / 2} \in B(\mathcal{H}) .
$$

This implies that $\operatorname{ad}_{a_{\chi}}^{\alpha}\left(k_{2} b^{-1}\right) \in B(\mathcal{H})$ using (12.5), by undoing the commutators with $k_{2}$, and using that $0 \notin \sigma(b)$. Therefore $k_{2} b^{-1} \in C^{2}\left(a_{\chi} ; \mathcal{H}\right)$. Since we saw that $k_{1} b^{-1} \in C^{2}\left(a_{\chi} ; \mathcal{H}\right)$ this also implies by [1, Proposition 5.2.3] that $b^{-1} k_{1} k_{2} b^{-1} \in$ $C^{2}\left(a_{\chi} ; \mathcal{H}\right)$. Hence $\left(\mathrm{M} 2^{\prime}\right)$ is satisfied.

The fact that condition (M3) is satisfied is also a standard result (one can either use pseudo-differential calculus or express $\langle b\rangle$ via almost-analytic extensions). Condition (M4) follows from (A2) since $k$ and $\langle s\rangle$ commute. Condition (M5) follows from the pseudo-differential calculus on scattering manifolds.

The fact that $\tau\left(b^{2}\right)=m_{\infty}^{2}$ follows from [15, Theorem 1]. This proves (2). 
From Proposition 12.4 and Proposition 7.4 we see that

$$
\left.\left.\sigma_{\mathrm{ess}}(H)=\sigma_{\mathrm{ess}}(\dot{H})=\right]-\infty,-m_{\infty}\right] \cup\left[m_{\infty},+\infty[.\right.
$$

12.1.2. Massless case. We consider $h_{0}$ as in (12.3) satisfying (12.4) but assume now that inf $\sigma\left(h_{0}\right)=0$. This is of course equivalent to $m_{\infty}=0$.

We assume $d \geq 3$, because the Hardy inequality on ( $\mathcal{M}, g)$ will play an important role. Instead of (12.5) we assume for $k=v$ that

$$
\left\{\begin{array}{l}
v(s, \omega)=v_{l}(s, \omega)+v_{s}(s, \omega), \\
\text { there exists } R_{0}>1,0 \leq \delta<1 \text { such that }\left|v_{l}(s, \omega)\right| \leq \delta \frac{d-2}{2}\langle s\rangle^{-1}, \text { for } s \geq R_{0}, \\
s v_{s}\langle p\rangle^{-1 / 2} \in B_{\infty}(\mathcal{H}), s^{3} v_{s}\langle p\rangle^{-1 / 2} \in B(\mathcal{H}) .
\end{array}\right.
$$

Note that compared to (12.5) we require an extra power of $s$ in the assumptions on $v_{2}$, which is needed to control the $|h|^{-\frac{1}{2}}$ or $b^{-1}$ term arising in (A1)-(A4) and (M1)-(M5), thanks to the Hardy inequality. As before $h=h_{0}-k^{2}$ is selfadjoint with domain $H^{2}(M)$, bounded below, $\langle h\rangle \sim\left\langle h_{0}\right\rangle$ and $\sigma_{\text {ess }}(h)=[0,+\infty[$. The operators $a$ and $\langle x\rangle$ are as in the previous subsection.

We have the following analog of Proposition 12.4, whose proof however is more involved and relies on estimates proved in Subsection A.1.

Proposition 12.5. Assume (12.3), (12.4) with $m_{\infty}=0$, (12.8), $\operatorname{Ker} h=\{0\}$ and $d \geq 3$. Then,

(1) conditions (E1)-(E3), (A1)-(A4), and (M1)-(M5) are satisfied;

(2) one has $\tau(b)=\{0\}$.

Proof. To check (E1)-(E3) we use again Proposition 12.1, with $\epsilon, r_{1}, r_{2}$ as in the proof of Proposition 12.4. We first claim that $v_{\mathrm{s}} \epsilon^{-1}$ is compact. We use

$$
\epsilon^{-1}=\langle\epsilon\rangle^{-1}+\langle\epsilon\rangle^{-1} \epsilon^{-1}(\langle\epsilon\rangle-\epsilon) .
$$

The term $v_{\mathrm{s}}(\epsilon+1)^{-1}$ is compact by (12.8), so it suffices to prove that $v_{\mathrm{s}}\langle\epsilon\rangle^{-1} \epsilon^{-1}$ is compact. We have

$$
v_{\mathrm{S}}\langle\epsilon\rangle^{-1} \epsilon^{-1}=\left(v_{\mathrm{S}}\langle s\rangle\langle\epsilon\rangle^{-1}\right) \times\left(\langle\epsilon\rangle\langle s\rangle^{-1}\langle\epsilon\rangle^{-1}\langle s\rangle\right) \times\left(\langle s\rangle \epsilon^{-1}\right) .
$$


The first factor is compact by (12.8). The second is seen to be bounded by commuting $\langle s\rangle^{-1}$ through $\langle\epsilon\rangle$. The third term is bounded by Proposition A.2 (1). Therefore $v_{\mathrm{s}} \epsilon^{-1}$ is compact. Since $\mathbb{1}_{s \leq R} v_{1}$ satisfies the same estimates as $v_{\mathrm{s}}$ we see that $r_{2} \epsilon^{-1} \in B_{\infty}(\mathcal{H})$. This also implies that $\left\|\mathbb{1}_{s \geq R} v_{\mathrm{s}} \epsilon^{-1}\right\| \rightarrow 0$ when $R \rightarrow \infty$. By Proposition A.2 (2) we obtain that $\left\|\mathbb{1}_{|x| \geq R} v_{1} \epsilon^{-1}\right\|<1$ for $R \gg 1$. Therefore $\left\|r_{1} \epsilon^{-1}\right\|<1$ for $R \gg 1$. Applying Proposition 12.1 we obtain (E1) and (E2). We also get that $|h| \sim \epsilon^{2} \sim D_{s}^{2}-\frac{1}{s^{2}} \Delta_{\mathcal{N}}$. By what we saw above, $k \epsilon^{-1}$ is bounded, hence (E3) also holds.

To check (A1)-(A4) we use again Lemma 7.10, with the same splitting of $k$ as in the proof of Proposition 12.4. We already checked that the hypotheses of Lemma 7.10 hold, which proves (A1)-(A4).

Condition (A3) is immediate since $k_{1}\langle\epsilon\rangle^{-1} \in B_{\infty}(\mathcal{H})$.

Condition (M1) and the fact that $k_{1}\langle b\rangle^{-1} \in C^{2}\left(a_{\chi}, \mathcal{H}\right)$ are proved as in Proposition 12.4. To prove (M2) we check the hypotheses of Lemma 8.4. To prove that $k_{2} b^{-1} \in C^{2}\left(a_{\chi} ; \mathcal{H}\right)$ we have to check that $\mathrm{ad}_{a_{\chi}}^{\alpha}\left(k_{2} b^{-1}\right)$ are bounded for $\alpha=1,2$. We use that

$$
\begin{gathered}
a_{\chi}\langle s\rangle^{-1}\langle p\rangle^{-1 / 2}, \quad\langle s\rangle^{-1}\langle p\rangle^{-1 / 2} b^{-1} a_{\chi}, \quad\langle s\rangle^{-1} b^{-1}, \\
a_{\chi} a_{\chi}\langle s\rangle^{-2}\langle p\rangle^{-1 / 2}, \quad\langle p\rangle^{-1 / 2}\langle s\rangle^{-2} b^{-1} a_{\chi} a_{\chi}
\end{gathered}
$$

are all in $B(\mathcal{H})$.

The bounds with $a_{\chi}$ are as in (12.7), using that $b^{-1} a_{\chi}=\tilde{\chi}\left(b^{2}\right) a \chi\left(b^{2}\right)$ for $\tilde{\chi} \in C_{0}^{\infty}(\mathbb{R})$, since $0 \notin \operatorname{supp} \chi$. The fact that $\langle x\rangle^{-1} b^{-1}$ is bounded follows from Proposition A.2 (1), using that $b^{2} \simeq \epsilon^{2}$. Undoing the commutators and using (12.8) we obtain that $\operatorname{ad}_{a_{\chi}}^{\alpha}\left(k_{2} b^{-1}\right)$ are bounded for $\alpha=1,2$. The same argument using that $k_{1} \in O\left(\langle s\rangle^{-1}\right)$ shows that $a d_{a_{\chi}}\left(b^{-1} k_{1} k_{2} b^{-1}\right)$ is bounded. This completes the proof of $\left(\mathrm{M} 2^{\prime}\right)$.

As in the massive case we prove that (M3) and (M4) hold and that $\tau\left(b^{2}\right)=\{0\}$ using [15, Theorem 1]. Condition (M5) follows from the pseudo-differential calculus on scattering manifolds.

As in Subsection 12.1.1 one has

$$
\sigma_{\mathrm{ess}}(H)=\sigma_{\mathrm{ess}}(\dot{H})=\mathbb{R} .
$$

12.1.3. Some additional remarks in the euclidean case. If $\mathcal{M}=\mathbb{R}^{d}$ and the metric $g$ is asymptotically flat, then using polar coordinates we see that $(\mathcal{M}, g)$ is a scattering manifold. In this case, using results of [21], it is possible to exclude eigenvalues and critical points embedded in the essential spectrum. 
Proposition 12.6. Assume that $\mathcal{M}=\mathbb{R}^{d}$ and $g$ is asymptotically flat. Assume moreover that $v=v_{1}+v_{2}$ where

$$
\left\{\begin{array}{l}
\partial_{x}^{\alpha} v_{1} \in O\left(\langle x\rangle^{-\mu-|\alpha|}\right), \quad \mu>0,|\alpha| \leq 2, \\
v_{2} \text { has compact support, } v_{2} \in L^{d}\left(\mathbb{R}^{d}\right) .
\end{array}\right.
$$

Then

$$
\sigma_{\mathrm{p}}(H) \cup c(H) \subset\left[-m_{\infty}, m_{\infty}\right] .
$$

If $m_{\infty}=0$ and $\operatorname{Ker} h=\{0\}$, then

$$
\sigma_{\mathrm{p}}(H) \cup c(H)=\emptyset .
$$

Proof. Since $\dot{\varepsilon}$ is a Pontryagin space, we know that critical points of $H$ are eigenvalues. From [11, Proposition 3.1] we know that $\sigma_{\mathrm{p}}(H) \subset\left[-m_{\infty}, m_{\infty}\right]$. Moreover Ker $h=\{0\}$ implies that Ker $H=\{0\}$.

12.2. Models with hyperbolic ends. We fix a smooth compact manifold $\mathcal{N}$, whose elements will be denoted by $\omega$ and a smooth positive density on $\mathcal{N}$ denoted by $d \omega$. We set $\mathcal{M}:=\mathbb{R} \times \mathcal{N}$, whose elements are denoted by $(s, \omega)$ and equip $\mathcal{M}$ with the density $d s d \omega$.

In this subsection we will describe some examples of Klein-Gordon equations

$$
\left(\partial_{t}-\mathrm{i} k\right)^{2} \phi(t)+h_{0} \phi(t)=0,
$$

on the Hilbert space $\mathcal{H}=L^{2}(\mathcal{M}, d s d \omega)$, to which the results of Sections 7 and 8 can be applied.

Remark 12.7. All the results of this subsection extend easily to the case where the smooth manifold $\mathcal{N}$ is equal to $\mathcal{M}_{0} \cup\left[1,+\infty\left[\times \mathcal{N}\right.\right.$, where $\mathcal{M}_{0}$ is compact. One has to assume that the restriction of $h_{0}$ to $[1,+\infty[\times \mathcal{N}$ satisfies similar assumptions as below, and the restriction of $h_{0}$ to $\mathcal{M}_{0}$ is a second order, elliptic differential operator with smooth coefficients.

We introduce the spaces of exponentially decreasing functions

$$
T^{p}(\mathcal{M}):=\left\{f \in C^{\infty}(\mathcal{M}): \partial_{s}^{\alpha} \partial_{\omega}^{\beta} f \in O\left(\mathrm{e}^{p|s|}\right)\right\}, \quad p \in \mathbb{R} .
$$

Similarly to Subsection 12.1.1, we set

$$
S^{p}(\mathcal{M}):=\left\{f \in C^{\infty}(\mathcal{M}): \partial_{s}^{\alpha} \partial_{\omega}^{\beta} f \in O\left(\langle s\rangle^{p-|\alpha|}\right)\right\}, \quad p \in \mathbb{R} .
$$


As usual a function $f$ in $T^{p}(\mathcal{M})$ resp. in $S^{p}(\mathcal{M})$ is called elliptic if $f^{-1} \in T^{-p}(\mathcal{M})$ resp. $f^{-1} \in S^{-p}(\mathcal{M})$.

We fix a second order differential operator $P=P\left(\omega, \partial_{\omega}\right)$ on $\mathcal{N}$, assumed to be self-adjoint, positive on $L^{2}(\mathcal{N}, d \omega)$ with domain $H^{2}(\mathcal{N})$.

We consider an operator $h_{0}$ acting on $C_{0}^{\infty}(\mathcal{M})$ as

$$
h_{0}=-c_{0}(s, \omega) \partial_{s} g_{0}(s, \omega) \partial_{s} c_{0}(s, \omega)-c_{-\frac{1}{2}}(s, \omega) P\left(\omega, \partial_{\omega}\right) c_{-\frac{1}{2}}(s, \omega)+d_{0}(s, \omega),
$$

where the coefficients $c_{0}, g_{0}, c_{-\frac{1}{2}}$ and $d_{0}$ satisfy

$$
\left\{\begin{array}{l}
c_{0}, g_{0} \text { elliptic in } S^{0}(\mathcal{M}), c_{0}-1, g_{0}-1 \in S^{-2}(\mathcal{M}), \\
c_{-\frac{1}{2}} \text { elliptic in } T^{-\frac{1}{2}}(\mathcal{M}),\langle s\rangle^{-2}\left(c_{-\frac{1}{2}}(s, \omega)-\tilde{c}_{-\frac{1}{2}}(s)\right) \in T^{-1 / 2}(\mathcal{M}) \\
\text { for some } \tilde{c}_{-\frac{1}{2}}(s) \text { elliptic in } T^{-\frac{1}{2}}(\mathcal{M}), \\
d_{0}(s, \omega) \in S^{0}(\mathcal{M}), d_{0}(s, \omega)-m_{\infty}^{2} \in S^{-2}(\mathcal{M}), m_{\infty} \geq 0
\end{array}\right.
$$

We assume moreover that on $C_{0}^{\infty}(\mathbb{R})$ one has

$$
h_{0} \geq m^{2}(s, \omega) \text { for some } m \in S^{0}(\mathcal{N}), m(s, \omega)>0, \quad \text { for all }(s, \omega) \in \mathcal{M} . \quad(12.15)
$$

It is easy to see that $h_{0}$ belongs to the general class studied in [7], [13, Section 3.3]. Therefore $h_{0}$ is self-adjoint, bounded below with domain

$$
\operatorname{Dom} h_{0}=\left\{u \in L^{2}(\mathcal{M}): h_{0} u \in L^{2}(\mathcal{M})\right\}=\operatorname{Dom} h_{\text {sep }},
$$

where

$$
h_{\text {sep }}=-\partial_{s}^{2}-\tilde{c}_{-\frac{1}{2}}(s) P\left(\omega, \partial_{\omega}\right) \tilde{c}_{-\frac{1}{2}}(s),
$$

is separable. Moreover the inequality (12.15) still holds on Dom $h_{0}$. One also knows that $\sigma_{\text {ess }}\left(h_{0}\right)=\left[m_{\infty}^{2},+\infty\left[\right.\right.$ where $m_{\infty}$ is defined in (12.14).

Concerning the operator $k$ we assume that $k=k(s, \omega)$ is a multiplication operator with

$$
k(s, \omega) \in S^{-2}(\mathcal{M}), k(s, \omega) m(s, \omega)^{-1} \rightarrow 0 \text { when } s \rightarrow \infty .
$$

From [7] we get that $h_{0}$ is self-adjoint on Dom $h_{\text {sep }}$. Since equation (12.15) implies Ker $h_{0}=\{0\}$, we see that we are dealing with a massive Klein-Gordon equation if and only if $m_{\infty}>0$. 
We now describe the conjugate operator $a$, following [7] and [13]. Let us fix functions $F, \chi \in C^{\infty}(\mathbb{R})$, with $F^{\prime}, \chi^{\prime} \geq 0, F(\lambda)=0$ for $\lambda \leq-1, F(\lambda)=1$ for $\lambda \geq-\frac{1}{2}, \chi(s)=0$ for $s \leq 1, \chi(s)=1$ for $s \geq 2$. We set

$$
F_{S}(\lambda)=F\left(S^{-1} \lambda\right) \quad \text { and } \quad \chi_{R}(s)=\chi\left(R^{-1} s\right) \quad \text { for } S, R \geq 1,
$$

and

$$
\begin{aligned}
X_{S, R}(s, P)=\chi_{R}^{2}(s) F_{S}^{2}(\sigma s-\ln (P+1))(\sigma s-\ln (P+1)+2 S) & \\
& +\chi_{R}^{2}(-s) F_{S}^{2}(-\sigma s-\ln (P+1))(\sigma s+\ln (P+1)-2 S), \\
a_{S, R}:= & \frac{1}{2}\left(X_{S, R}(s, P) D_{s}+D_{s} X_{S, R}(s, P)\right) .
\end{aligned}
$$

Let us summarize some properties of $h, a_{S, R}$, which can be proved as in [7] and in [13].

Proposition 12.8. Assume (12.14), (12.15) and (12.16). Then

(1) $a_{S, R}$ is essentially self-adjoint on $\operatorname{Dom}\left(h_{\mathrm{sep}}+\langle s\rangle^{2}\right)$;

(2) $\langle s\rangle^{-p} a_{S, R}\langle s\rangle^{p-1} \in B(\mathcal{H})$, for $p \in \mathbb{R}$;

(3) $h_{0}+f \in C^{2}\left(a_{S, R}\right)$, for any $f \in S^{-2}(\mathcal{M})$;

(4) let

$$
\tau\left(h_{0}+f\right):=\bigcap_{S, R \geq 1} \tau\left(h_{0}+f, a_{S, R}\right)
$$

(see the beginning of Subsection 8.3 for notation). Then

$$
\tau\left(h_{0}+f\right)=\left\{m_{\infty}^{2}\right\} .
$$

Remark 12.9. Note that (4) means that if $\lambda \neq m_{\infty}^{2}$, then there exist an interval $I$ with $\lambda \in I$, parameters $S, R \geq 1$, a constant $c_{0}>0$ and $K \in B_{\infty}(\mathcal{H})$ such that

$$
\mathbb{1}_{I}\left(h_{0}+f\right)\left[h_{0}+f, \mathrm{i} a_{S, R}\right] \mathbb{1}_{I}\left(h_{0}+f\right) \geq c_{0} \mathbb{1}_{I}\left(h_{0}+f\right)+K .
$$

In the sequel we will forget the fact that the scalar conjugate operator $a_{S, R}$ depends on parameters $S, R$, and denote it simply by $a$.

It remains to fix the reference weight appearing in hypothesis (M3). We choose

$$
\langle x\rangle:=\left(s^{2}+1\right)^{\frac{1}{2}} .
$$

To prove Proposition 12.11 below, we will need the following lemma, whose proof may be found in Subsection A.4. 
Lemma 12.10. Let $f \in S^{-2}(\mathcal{M})$ such that $\epsilon^{2}-f^{2}=: b^{2} \geq 0$. Then

$$
\left[\langle b\rangle,\langle s\rangle^{\delta}\right] \in B(\mathcal{H}), \quad \text { for } 0 \leq \delta \leq 1 .
$$

Proposition 12.11. Assume (12.14)-(12.16) and $\operatorname{Ker} h=\{0\}$. Then

(1) conditions (E1)-(E3), (A1)-(A4), and (M1)-(M5) are satisfied;

(2) one has $\tau(b)=\left\{m_{\infty}\right\}$.

Proof. Set

$$
\epsilon=h_{0}^{\frac{1}{2}}
$$

We first claim that

$$
\left\{\begin{array}{l}
\text { (1) }\left\|\mathbb{1}_{\{|s| \geq R\}} k \epsilon^{-1}\right\| \rightarrow 0 \text { when } R \rightarrow+\infty, \\
\text { (2) } \mathbb{1}_{\{|s| \leq R\}} k \epsilon^{-1} \in B_{\infty}(\mathcal{H}) .
\end{array}\right.
$$

In fact (1) follows from the fact that $h_{0} \geq m^{2}(s, \omega)$ and $k(s, \omega) m(s, \omega)^{-1} \rightarrow 0$ when $s \rightarrow \infty$, using also Kato-Heinz inequality. To complete the proof of (12.17) it suffices to prove that $g(s) \epsilon^{-1} \in B_{\infty}(\mathcal{H})$ for $g \in C_{0}^{\infty}(\mathbb{R})$. Note that $g(s) \epsilon^{-1}$ is bounded by (12.15).

As in the proof of Proposition 12.5, it suffices, using (12.9), to check that both $g(s)\langle\epsilon\rangle^{-1}$ and $g(s)\langle\epsilon\rangle^{-1} \epsilon^{-1}$ are compact. The term $g(s)\langle\epsilon\rangle^{-1}$ is compact, by [7, Lemma 1.2]. We write the second term as $\langle\epsilon\rangle^{-1} g(s) \epsilon^{-1}-\left[\langle\epsilon\rangle^{-1}, g(s)\right] \epsilon^{-1}$. The first term is again compact since $g(s) \epsilon^{-1}$ is bounded. We write the second term as

$$
\left[\langle\epsilon\rangle^{-1}, g(s)\right] \epsilon^{-1}=\frac{\mathrm{i}}{2 \pi} \int_{\mathbb{C}} \frac{\partial \tilde{f}_{-\frac{1}{2}}}{\partial \bar{z}}(z)\left(z-\epsilon^{2}\right)^{-1}\left[\epsilon^{2}, g\right] \epsilon^{-1}\left(z-\epsilon^{2}\right)^{-1} d z \wedge d \bar{z},
$$

where $\tilde{f}_{-\frac{1}{2}}$ is an almost analytic extension of

$$
f_{-\frac{1}{2}}(\lambda)=\left(\lambda^{2}+1\right)^{-1 / 4},
$$

satisfying

$$
\left\{\begin{array}{l}
\operatorname{supp} \tilde{f}_{-\frac{1}{2}} \subset\{z \in \mathbb{C}:|\operatorname{Im} z| \leq C\langle\operatorname{Re} z\rangle\}, \\
\left|\frac{\partial \tilde{f}_{-\frac{1}{2}}}{\partial \bar{z}}(z)\right| \leq C_{N}\langle z\rangle^{-3 / 2-N}|\operatorname{Imz}|^{N}, \quad N \in \mathbb{N} .
\end{array}\right.
$$


Since $\epsilon^{2}$ is a second order differential operator, we obtain $\left[\epsilon^{2}, g\right]=\left(\epsilon^{2}+1\right) B \tilde{g}(s)$ with $B$ is compact and $\tilde{g} \in C_{0}^{\infty}(\mathbb{R})$. Therefore $\left(\epsilon^{2}+1\right)^{-1}\left[\epsilon^{2}, g\right] \epsilon^{-1}$ is compact. We use now the bounds

$$
\left\|\left(\epsilon^{2}-z\right)^{-1}\right\| \in O\left(|\operatorname{Im} z|^{-1}\right), \quad\left\|\left(\epsilon^{2}-z\right)^{-1}\left(\epsilon^{2}+1\right)\right\| \in O\left(\langle z\rangle|\operatorname{Im} z|^{-1}\right),
$$

and (12.18) to obtain that $\left[\langle\epsilon\rangle^{-1}, g(s)\right] \epsilon^{-1}$ is compact. This completes the proof of (12.17). We apply then Proposition 12.1 with $r_{1}=\mathbb{1}_{\{|s| \geq R\}} k, r_{2}=\mathbb{1}_{\{|s| \leq R\}} k$. The hypotheses of Proposition 12.1 hold by (12.17), which implies (E1) and (E2). Moreover $|h| \sim h_{0}$ and we can replace $|h|^{-\frac{1}{2}}$ by $\epsilon^{-1}$ in condition (E3) and conditions (A1)-(A4). Since by (12.17) we know that $k \epsilon^{-1} \in B(\mathcal{H})$, condition (E3) holds. To check (A1)-(A4) we use Lemma 7.10 and split $k$ as $k_{1}+k_{2}$ with

$$
k_{1}=F_{\infty}\left(R^{-1}|s|\right) v_{1}, k_{2}=v_{\mathrm{s}}+F_{0}\left(R^{-1}|s|\right) v_{1}
$$

for some $F_{0}, F_{\infty}$ as in (12.6). By (12.17) $\left\|k_{1} \epsilon^{-1}\right\|<1$ for $R \gg 1, k_{2} \epsilon^{-1}$ is compact. The fact that $k\langle\epsilon\rangle^{-1}$ is compact follows once again from [7, Lemma 1.2].

Let us now check (M1) and (M2'). Note first that $b^{2}=\epsilon^{2}-k_{1}^{2}$ is of the form $\epsilon^{2}+f$ for $f \in S^{-2}(\mathcal{M})$. Moreover if $R \gg 1$ we have

$$
b^{2} \geq \frac{1}{2} m^{2}(s, \omega),
$$

by (12.15). Then (M1) follows by Proposition 12.8 (3). By Proposition 12.8 (2), and observing that $k_{1} \in S^{-2}(\mathcal{N})$, we obtain that $k_{1} \in C^{2}\left(a_{\chi} ; \mathcal{H}\right)$ by undoing the commutators. Since $\langle b\rangle^{-1} \in C^{2}\left(a_{\chi} ; \mathcal{H}\right)$, this proves the first condition of $\left(\mathrm{M} 2^{\prime}\right)$.

To prove the rest of $\left(\mathrm{M} 2^{\prime}\right)$ we claim that $g(s) b^{-1} \in C^{2}\left(a_{\chi} ; \mathcal{H}\right)$ for $g \in C_{0}^{\infty}(\mathbb{R})$. Note that this implies the last two conditions of (M2'), since $k_{2}, k_{1} k_{2} \in C_{0}^{\infty}(\mathbb{R})$.

If $m_{\infty}>0$ this is proved by the same argument as before. If $m_{\infty}=0$, i.e. we are considering the massless case, then we argue as in the proof of Lemma 12.5: we use that

$$
\begin{gathered}
a_{\chi}\langle s\rangle^{-1}, \quad\langle s\rangle b^{-1} a_{\chi}, \quad\langle s\rangle^{n} g(s) b^{-1}, \\
a_{\chi} a_{\chi}\langle s\rangle^{-2}, \quad\langle s\rangle^{-2} b^{-1} a_{\chi} a_{\chi}
\end{gathered}
$$

are in $B(\mathcal{H})$.

The bounds with $a_{\chi}$ rely on Proposition 12.8 (2) and the fact that

$$
b^{-1} a_{\chi}=\tilde{\chi}\left(b^{2}\right) a \chi\left(b^{2}\right)
$$

for $\tilde{\chi} \in C_{0}^{\infty}(\mathbb{R})$ since $0 \notin \operatorname{supp} \chi$. As before we complete the proof by undoing the commutators with $g(s) b^{-1}$. 
We now prove (M3). The first condition of equation (M3) follows by Proposition 12.8 (2). To prove the second it suffices to prove that

$$
\left[\langle b\rangle,\langle s\rangle^{\delta}\right] \in B(\mathcal{H}), \quad 0 \leq \delta \leq 1,
$$

which has been shown in Lemma 12.10. Finally (M4) is true since $k$ and $\langle x\rangle$ commute, and the fact that $\tau(b)=\left\{m_{\infty}\right\}$ follows from Proposition 12.8 (4).

\section{Appendix A}

A.1. Diamagnetic and Hardy inequalities. We start by recalling some wellknown facts related to the diamagnetic inequality. We are working on the scattering manifolds introduced in Subsection 12.1 and set

$$
p=-\sum_{j, k=1}^{d} g^{-1 / 4} \partial_{j} g^{1 / 2} g^{j k} \partial_{k} g^{-1 / 4},
$$

and

$$
p_{A}:=-\sum_{j, k=1}^{d} g^{-1 / 4}\left(\partial_{j}-\mathrm{i} A_{j}\right) g^{1 / 2} g^{j k}\left(\partial_{k}-\mathrm{i} A_{k}\right) g^{-1 / 4}
$$

where $A(s, \omega)$ satisfies (12.4). We use the notations of Subsection 12.1 .

Lemma A.1. Let $V \in C^{0}\left(\mathbb{R}^{d}, \mathbb{R}\right)$ be a bounded potential. Then

$$
p+V \geq 0 \Longrightarrow p_{A}+V \geq 0,
$$

Proof. Let us first recall the diamagnetic inequality

$$
\left|\mathrm{e}^{-t\left(p_{A}+V\right)} u\right| \leq \mathrm{e}^{-t(p+V)}|u|, \quad u \in \mathcal{H}, t \geq 0 .
$$

This inequality is well known on $\mathbb{R}^{d}$ and also holds on scattering manifolds. Indeed it is equivalent to a certain estimate on the quadratic forms associated to the operators, which clearly also holds on scattering manifolds, see [31] for details. Now recall that

$$
a^{-\alpha}=C_{\alpha} \int_{0}^{+\infty} t^{\alpha-1} \mathrm{e}^{-t a} d t, \quad a>0, \alpha>0,
$$

where $C_{\alpha}$ is a positive constant. Using (A.2) for $\alpha=1$ we obtain

$$
\left.\left(u \mid p_{A}+V+\delta\right)^{-1} u\right) \leq\left(\left|u \|(p+V+\delta)^{-1}\right| u \mid\right) \leq \delta^{-1}\|u\|^{2},
$$

which by Kato-Heinz inequality implies that $p_{A}+V+\delta \geq \delta$, which proves the lemma. 
We now prove some estimates related to Hardy's inequality on the scattering manifold ( $\mathcal{M}, g$ ) considered in Subsection 12.1.

Let $g^{0}$ be a conic metric as in Definition 12.2, restricted to $\left.\mathcal{M}_{\infty}=\right] 1,+\infty[\times \mathcal{N}$. The corresponding Laplace-Beltrami operator is

$$
-\Delta_{g} 0=-s^{(1-d)} \partial_{s} s^{d-1} \partial_{s}-\Delta_{h},
$$

which is self-adjoint on $L^{2}\left(\mathcal{M}_{\infty}, s^{d-1}|h|^{\frac{1}{2}} d s d \omega\right)$. The usual proof on $\mathbb{R}^{d}$, which relies on the identity $s^{-2}=-\frac{1}{2} s \partial_{s}\left(s^{-2}\right)$ for $s=|x|$, yields, for $u \in C_{0}^{\infty}\left(\mathcal{M}_{\infty}\right)$,

$$
\left(\frac{d-2}{2}\right)^{2} \int_{\mathcal{M}_{\infty}} s^{-2}|u|^{2} s^{d-1}|h|^{\frac{1}{2}} d s d \omega \leq-\int_{\mathcal{M}_{\infty}} \bar{u} \Delta_{g^{0}} u s^{d-1}|h|^{\frac{1}{2}} d s d \omega,
$$

where $-\Delta_{h}$ is the Laplace-Beltrami operator on $(\mathcal{N}, h)$. Using the unitary map

$$
T: L^{2}\left(\mathcal{M},|g|^{\frac{1}{2}} d s d \omega\right) \ni u \longmapsto|g|^{1 / 4} u \in L^{2}(\mathcal{M}, d s d \omega),
$$

this immediately implies that ([33, Proposition 3.4])

$$
s^{-2} \leq C p, C>0, \text { on } \mathcal{H}=L^{2}(\mathcal{M}, d s d \omega) .
$$

Proposition A.2. Assume (12.4) and $d \geq 3$. Then

(1) $p_{A} \geq C\langle s\rangle^{-2}$ and

(2) if in addition $v_{l}(x)$ satisfies (12.8), then

$$
(1+\alpha)^{-1} p_{A} \geq F_{\infty}^{2}\left(R^{-1}|s|\right) v_{l}^{2}(s), \quad \text { for some } 0<\alpha<1, R \gg 1,
$$

and $F_{\infty}$ as in (12.6).

Proof. Statement (1) follows from (A.4) and Lemma A.1. Let us now prove (2). Since $g$ is a long-range perturbation of $g^{0}$, we deduce from (A.3) that

$$
\mathbb{1}_{|s| \geq R} \leq\left(\frac{2}{d-2}\right)^{2}\left(1+O\left(R^{-\mu}\right)\right) p,
$$

hence, by Lemma A.1,

$$
\mathbb{1}_{\{s \geq R\}}(s)\langle s\rangle^{-2} \leq\left(\frac{2}{d-2}\right)^{2}\left(1+O\left(R^{-\mu}\right)\right) p_{A},
$$

which implies (2), using the estimate (12.8) on $v_{l}(s, \omega)$. 
A.2. Proof of Proposition 12.1. Let $\epsilon_{1}^{2}=\epsilon^{2}-r_{1}^{2}$. Since $\left\|r_{1} \epsilon^{-1}\right\|<1$, we have $\epsilon_{1}^{2} \sim \epsilon^{2}$. Therefore $\operatorname{Ker} \epsilon_{1}=\{0\}$, and $r_{2} \epsilon_{1}^{-1} \in B_{\infty}(\mathcal{H})$. We have

$$
h=\epsilon^{2}-r^{2}=\epsilon_{1}^{2}-r_{2}^{2} .
$$

Therefore denoting $\epsilon_{1}$ again by $\epsilon$ we can assume that $r_{1}=0$ and denote $r_{2}$ by $r$, so that $r \epsilon^{-1} \in B_{\infty}(\mathcal{H}), h=\epsilon^{2}-r^{2}$. Note that $\sigma_{\text {ess }}(h)=\sigma_{\text {ess }}\left(\epsilon^{2}\right)$. If $m>0$ then $\sigma_{\text {ess }}(h) \subset\left[m_{\infty}^{2},+\infty\left[\right.\right.$ for some $m_{\infty}>0$ hence $\operatorname{Tr} \mathbb{1}_{-\infty, 0]}(h)<\infty$. Moreover $0 \notin \sigma(h)$ and $0 \notin \sigma\left(\epsilon^{2}\right)$, thus $|h| \sim\langle h\rangle \sim\langle\epsilon\rangle^{2} \sim \epsilon^{2}$. Hence (2) in (12.1) also holds.

Let us now assume that $m=0$. We first prove (1) from (12.1). Noting that $r\langle\epsilon\rangle^{-1}$ is bounded, we obtain by the Birman-Schwinger principle that

$$
\operatorname{Tr} \mathbb{1}_{-\infty,-\alpha]}(h)=\operatorname{Tr} \mathbb{1}_{] 1,+\infty[}\left(K_{\alpha}\right),
$$

for $K_{\alpha}=r\left(\epsilon^{2}+\alpha\right)^{-1} r \in B_{\infty}(\mathcal{H}), \alpha>0$. Since $r \epsilon^{-1} \in B_{\infty}(\mathcal{H})$ we have $K_{\alpha} \nearrow K_{0}=r \epsilon^{-2} r \in B_{\infty}(\mathcal{H})$, hence

$$
\operatorname{Tr} \mathbb{1}_{]-\infty, 0[}(h)=\operatorname{Tr} \mathbb{1}_{] 1,+\infty[}\left(K_{0}\right)<\infty .
$$

Since $\operatorname{Ker} h=\{0\}$, this implies that $\operatorname{Tr} \mathbb{1}_{-\infty, 0]}(h)<\infty$, which proves (1) in (12.1).

We now prove (2) from (12.1). Set

$$
P_{ \pm}:=\mathbb{1}_{\mathbb{R}^{ \pm}}(h) .
$$

If

$$
h u=\left(\epsilon^{2}-r^{2}\right) u=-\lambda u, \quad \lambda>0,
$$

then we have

$$
\epsilon^{-1} u=(-\lambda)^{-1}\left(\mathbb{1}-\epsilon^{-1} r^{2} \epsilon^{-1}\right) \epsilon u \in \mathcal{H} .
$$

This implies that

$$
\mid u)\left(u \mid \leq C \epsilon^{2}, \quad C>0 .\right.
$$

Since $\operatorname{Tr} P_{-}<\infty$ this implies that $P_{-} \leq C \epsilon^{2}$, for some $C>0$. Now

$$
|h|=h-2 h P_{-} \leq h+2|\inf \sigma(h)| P_{-} \leq \epsilon^{2}+2 C|\inf \sigma(h)| \epsilon^{2},
$$

which shows that $|h| \leq C \epsilon^{2}$ for some $C>0$.

To prove the lower bound, we adapt some arguments in [32]. Let

$$
h_{\delta}=h-\delta r^{2}=\epsilon^{2}-(1+\delta) r^{2} .
$$

Again by the Birman-Schwinger principle, we have $\operatorname{Tr} \mathbb{1}_{]-\infty, 0}\left(h_{\delta}\right)=\operatorname{Tr} \mathbb{1}_{-\infty, 0[}(h)$, for $\delta$ small enough. Therefore there exists $c_{0}, \delta_{0}>0$ such that

$$
\mathbb{1}_{\left[-c_{0}, 0[\right.}\left(h_{\delta}\right)=0, \quad \text { for all } 0 \leq \delta \leq \delta_{0} .
$$


We fix cutoff functions $\chi_{ \pm}$with $\chi_{-} \in C_{0}^{\infty}(]-\infty,-c_{0} / 2[), \chi_{+} \in C^{\infty}(]-c_{0},+\infty[)$ and $\chi_{-}^{2}(h)+\chi_{-}^{2}(h)=1$. From (A.5) it follows that $\chi_{+}\left(h_{\delta}\right) h_{\delta} \chi_{+}\left(h_{\delta}\right) \geq 0$. Therefore,

$$
\begin{aligned}
P_{+} h_{\delta} P_{+} & =P_{+}\left(\chi_{-}\left(h_{\delta}\right) h_{\delta} \chi_{-}\left(h_{\delta}\right)+\chi_{+}\left(h_{\delta}\right) h_{\delta} \chi_{+}\left(h_{\delta}\right)\right) P_{+} \\
& \geq P_{+}\left(\chi_{-}\left(h_{\delta}\right) h_{\delta} \chi_{-}\left(h_{\delta}\right) P_{+}\right. \\
& =P_{+} R h_{\delta} R P_{+},
\end{aligned}
$$

for $R=\chi_{-}\left(h_{\delta}\right)-\chi_{-}(h)$, using that $P_{+} \chi_{-}(h)=0$.

We claim that

$$
R h_{\delta} R \geq-C \delta^{2} \epsilon^{2}, \quad C>0, \text { uniformly for } 0 \leq \delta \leq \delta_{0},
$$

which follows from

$$
\left\|\epsilon^{-1} R\left\langle h_{\delta}\right\rangle^{\frac{1}{2}}\right\| \leq C \delta, \quad \text { uniformly for } 0 \leq \delta \leq \delta_{0} .
$$

To prove (A.8) it suffices to check that

$$
\left\|(\epsilon+\alpha)^{-1} R\left\langle h_{\delta}\right\rangle^{\frac{1}{2}}\right\| \leq C \delta, \quad \text { uniformly for } 0 \leq \delta \leq \delta_{0}, \alpha>0 .
$$

We have

$$
\begin{aligned}
(\epsilon & +\alpha)^{-1} R\left\langle h_{\delta}\right\rangle^{\frac{1}{2}} \\
& =\delta \frac{\mathrm{i}}{2 \pi} \int_{\mathbb{C}} \frac{\partial}{\partial \bar{z}} \tilde{\chi}_{-}(z)(\epsilon+\alpha)^{-1}(z-h)^{-1} r^{2}\left(z-h_{\delta}\right)^{-1}\left\langle h_{\delta}\right\rangle^{\frac{1}{2}} d z \wedge d \bar{z},
\end{aligned}
$$

where $\tilde{\chi}_{-}(z) \in C_{0}^{\infty}(\mathbb{R})$ is an almost analytic extension of $\chi_{-}$. We write

$$
\begin{aligned}
& r l(\epsilon+\alpha)^{-1}(z-h)^{-1} r^{2}\left(z-h_{\delta}\right)^{-1}\left\langle h_{\delta}\right\rangle^{\frac{1}{2}} \\
&=(z-h)^{-1}(\epsilon+\alpha)^{-1} r^{2}\left(z-h_{\delta}\right)^{-1}\left\langle h_{\delta}\right\rangle^{\frac{1}{2}} \\
&+(z-h)^{-1}\left[h,(\epsilon+\alpha)^{-1}\right](z-h)^{-1} r^{2}\left(z-h_{\delta}\right)^{-1}\left\langle h_{\delta}\right\rangle^{\frac{1}{2}} \\
&=(z-h)^{-1}(\epsilon+\alpha)^{-1} r^{2}\left(z-h_{\delta}\right)^{-1}\left\langle h_{\delta}\right\rangle^{\frac{1}{2}} \\
&+(z-h)^{-1}(\epsilon+\alpha)^{-1} r^{2}(z-h)^{-1} r^{2}\left(z-h_{\delta}\right)^{-1}\left\langle h_{\delta}\right\rangle^{\frac{1}{2}} \\
&-(z-h)^{-1} r^{2}(\epsilon+\alpha)^{-1}(z-h)^{-1} r^{2}\left(z-h_{\delta}\right)^{-1}\left\langle h_{\delta}\right\rangle^{\frac{1}{2}} \\
&=: I_{1}(z)+I_{2}(z)-I_{3}(z) .
\end{aligned}
$$


We write

$$
\begin{aligned}
I_{1}(z) & =(z-h)^{-1} \times(\epsilon+\alpha)^{-1} r^{2}\left\langle h_{\delta}\right\rangle^{-\frac{1}{2}} \times\left(z-h_{\delta}\right)^{-1}\left\langle h_{\delta}\right\rangle \\
& =O\left(|\operatorname{Im} z|^{-2}\right), \quad \text { uniformly in } \alpha, \delta \text { and } z \in \operatorname{supp} \tilde{\chi}_{-},
\end{aligned}
$$

using that $r \epsilon^{-1}$ is bounded, and $r\left\langle h_{\delta}\right\rangle^{-\frac{1}{2}}$ is bounded uniformly in $0 \leq \delta \leq \delta_{0}$. Similarly we have

$$
\begin{aligned}
I_{2}(z)= & (z-h)^{-1} \times(\epsilon+\alpha)^{-1} r^{2}\langle h\rangle^{-\frac{1}{2}} \times\langle h\rangle(z-h)^{-1} \\
& \times\langle h\rangle^{-\frac{1}{2}} r^{2}\left\langle h_{\delta}\right\rangle^{-\frac{1}{2}} \times\left(z-h_{\delta}\right)^{-1}\left\langle h_{\delta}\right\rangle \\
= & O\left(|\operatorname{Im} z|^{-3}\right), \quad \text { uniformly in } \alpha, \delta \text { and } z \in \operatorname{supp} \tilde{\chi}_{-} .
\end{aligned}
$$

A similar argument shows that $I_{3}(z)$ satisfies the same bound as $I_{2}(z)$. Therefore using (A.10) we obtain (A.9), hence (A.7).

We have now

$$
\begin{aligned}
P_{+} h P_{+} & =(1+\delta)^{-1} p_{+} h_{\delta} P_{+}+\delta(1+\delta)^{-1} P_{+} \epsilon^{2} P_{+} \\
& \geq\left(\delta-C \delta^{2}\right)(1+\delta)^{-1} P_{+} \epsilon^{2} P_{+}
\end{aligned}
$$

by (A.6) and (A.7). Choosing $\delta$ small enough we obtain that

$$
P_{+} \epsilon^{2} P_{+} \leq C P_{+} h P_{+}, \quad C>0
$$

On the other hand since Ran $P_{-}$is finite dimensional and included in Dom $\epsilon$, we have

$$
P_{-} \epsilon^{2} P_{-} \leq C P_{-}^{2} \text { for some } C>0 \text {. }
$$

Using that

$$
P_{+} \epsilon^{2} P_{-}+P_{-} \epsilon^{2} P_{+} \leq P_{+} \epsilon^{2} P_{+}+P_{-} \epsilon^{2} P_{-}
$$

we finally obtain

$$
\begin{aligned}
\epsilon^{2} & =\left(P_{+}+P_{-}\right) \epsilon^{2}\left(P_{+}+P_{-}\right) \\
& \leq 2 P_{+} \epsilon^{2} P_{+}+2 P_{-} \epsilon^{2} P_{-} \\
& \leq 2 C P_{+} h P_{+}+2 C P_{-}^{2} \leq C^{\prime}|h|,
\end{aligned}
$$

where in the last inequality we used (A.5) for $\delta=0$. This proves (2) in (12.1). 
A.3. Proof of Lemma 8.2. Let $\tilde{f}_{-\delta}$ be an almost analytic extension of the function $\langle\cdot\rangle^{-\delta}$ satisfying

$$
\operatorname{supp} \tilde{f}_{-\delta} \subset\{z \in \mathbb{C}:|\operatorname{Im} z| \leq c\langle\operatorname{Re} z\rangle\},
$$

and

$$
\left|\frac{\partial \tilde{f}_{-\delta}}{\partial \bar{z}}(z)\right| \leq C_{N}\langle z\rangle^{-\delta-1-N}|\operatorname{Im} z|^{N}, \quad N \in \mathbb{N},
$$

see [4, Appendix C.2]. We have

$$
\begin{aligned}
& {\left[\left\langle a_{\chi}\right\rangle^{-\delta},\langle b\rangle-b\right]\left\langle a_{\chi}\right\rangle^{\delta}} \\
& \quad=\frac{\mathrm{i}}{2 \pi} \int_{\mathbb{C}} \frac{\partial \tilde{f}_{-\delta}}{\partial \bar{z}}(z)\left(z-a_{\chi}\right)^{-1}\left[a_{\chi},\langle b\rangle-b\right]\left(z-a_{\chi}\right)^{-1}\left\langle a_{\chi}\right\rangle^{\delta} d z \wedge d \bar{z} .
\end{aligned}
$$

Since $0 \notin \operatorname{supp} \chi$, there exist $g \in C_{0}^{\infty}(\mathbb{R})$ such that

$$
\left[a_{\chi},\langle b\rangle-b\right]=\chi\left(b^{2}\right)\left[a, g\left(b^{2}\right)\right] \chi\left(b^{2}\right) \in B(\mathcal{H}),
$$

by (M1). Now we use the bound $\left\|\left(z-a_{\chi}\right)^{-1}\left\langle a_{\chi}\right\rangle^{\delta}\right\| \in O\left(\langle z\rangle^{\delta}|\operatorname{Im} z|^{-1}\right)$ and the estimates satisfied by $\tilde{f}_{-\delta}$ to obtain that the integral in (A.14) is norm convergent. This completes the proof of the lemma.

A.4. Proof of Lemma 12.10. Note that $b^{2}$ is of the form (12.13). We claim first that

$$
\left[b^{2},\langle s\rangle^{\delta}\right]\langle b\rangle^{-1},\langle b\rangle^{-1}\left[b^{2},\left[b^{2},\langle s\rangle^{\delta}\right]\right]\langle b\rangle^{-1} \in B(\mathcal{H}), \quad 0 \leq \delta \leq 1 .
$$

In fact this follows by an easy computation using (12.14) and the fact that

$$
\partial_{s}\langle b\rangle^{-1}, c_{-\frac{1}{2}}(s, \omega) \partial_{\omega}\langle b\rangle^{-1} \in B(\mathcal{H}),
$$

see [13, Lemma 4.3.1].

We write now

$$
\langle b\rangle=\left(b^{2}+1\right)\langle b\rangle^{-1},
$$

and

$$
\left[\langle b\rangle,\langle s\rangle^{-\delta}\right]=\left[b^{2},\langle s\rangle^{-\delta}\right]\langle b\rangle^{-1}+\left(b^{2}+1\right)\left[\langle b\rangle^{-1},\langle s\rangle^{-\delta}\right] .
$$

The first term is bounded by (A.15). To estimate the second term, we introduce the function

$$
f_{-\frac{1}{2}}(\lambda)=(\lambda+1)^{-\frac{1}{2}}
$$


and write with $\tilde{f}_{-\frac{1}{2}}$ as in (12.18):

$$
\begin{aligned}
&\left(b^{2}+1\right)\left[\langle b\rangle^{-1},\langle s\rangle^{-\delta}\right] \\
&=\frac{\mathrm{i}}{2 \pi} \int_{\mathbb{C}} \frac{\partial \tilde{f}_{-\frac{1}{2}}}{\partial \bar{z}}(z)\left(b^{2}+1\right)\left(z-b^{2}\right)^{-1}\left[b^{2},\langle s\rangle^{\delta}\right]\left(z-b^{2}\right)^{-1} d z \wedge d \bar{z} \\
&=\left(b^{2}+1\right) f_{-\frac{1}{2}}^{\prime}\left(b^{2}\right)\left[b^{2},\langle s\rangle^{\delta}\right] \\
&+\frac{\mathrm{i}}{2 \pi} \int_{\mathbb{C}} \frac{\partial \tilde{f}_{-\frac{1}{2}}}{\partial \bar{z}}(z)\left(b^{2}+1\right)\left(z-b^{2}\right)^{-2}\left[b^{2},\left[b^{2},\langle s\rangle^{\delta}\right]\left(z-b^{2}\right)^{-1} d z \wedge d \bar{z} .\right.
\end{aligned}
$$

The first term is again bounded using (A.15) and the fact that $f_{-\frac{1}{2}}^{\prime}(\lambda) \in O\left(\langle\lambda\rangle^{-3 / 2}\right)$. The integral in the second term is norm convergent, using (A.15), the estimates on $\tilde{f}_{-\frac{1}{2}}$ and the bounds $\langle b\rangle^{\alpha}\left(z-b^{2}\right)^{-1} \in O\left(\langle z\rangle^{\alpha / 2}|\operatorname{Im} z|^{-1}\right)$ for $0 \leq \alpha \leq 2$. This completes the proof of the fact that $\left[\langle b\rangle,\langle s\rangle^{\delta}\right]$ is bounded.

\section{Appendix B}

In this appendix we prove Theorem 3.17. We first recall some standard results.

Let $X$ be a locally compact space, $C(X)$ the space of bounded continuous functions and $B(X)$ the space of bounded Borel functions. We recall that a sequence $\left(\varphi_{n}\right)_{n \in \mathbb{N}}$ in $B(X)$ is $b$-convergent to $\varphi$, written as $\mathrm{b}-\lim _{n} \varphi_{n}=\varphi$ if $\sup _{n}\left\|\varphi_{n}\right\|_{\infty}<\infty$ and $\varphi_{n} \rightarrow \varphi$ pointwise on $X$.

The monotone class theorem implies that $B(X)$ is the smallest space of functions on $X$ containing $C(X)$ and stable under bounded convergence of sequences. The Riesz theorem says that any continuous linear form on $C(X)$ uniquely extends to a linear form on $B(X)$ continuous for the b-convergence of sequences.

Recall also that a Banach space $\mathcal{H}$ is weakly sequentially complete, if for each sequence $\left(u_{n}\right)_{n \in \mathbb{N}}$ in $\mathcal{H}$ such that $\lim _{n}\left\langle f, u_{n}\right\rangle$ exists for each $f \in \mathcal{H}^{\#}$, there exists $u \in \mathcal{H}$ with $\lim _{n}\left\langle f, u_{n}\right\rangle=\langle f, u\rangle$ for each $f \in \mathcal{H}^{\#}$. Reflexive Banach spaces, hence Hilbertizable spaces, are weakly sequentially complete.

Let $\mathcal{H}$ be weakly sequentially complete. If $\left(T_{n}\right)_{n \in \mathbb{N}}$ is a sequence in $B(\mathcal{H})$ such that $\lim _{n}\left\langle f, T_{n} u\right\rangle$ exists for each $u \in \mathcal{H}, f \in \mathcal{H}^{\#}$, then there is a unique $T \in B(\mathcal{H})$ such that $\mathrm{w}-\lim _{T_{n}}=T$.

From these facts, it is straightforward to prove the following result, see e.g. [36, Corollary 9.1.10]. 
Theorem B.1. Let $X$ a locally compact space, $\mathcal{H}$ a weakly sequentially complete Banach space. Then if $F_{0}: C(X) \rightarrow B(\mathcal{H})$ is a continuous algebra morphism, there is a unique algebra morphism $F: B(X) \rightarrow B(\mathcal{H})$ such that $\mathrm{b}-\lim _{n} \varphi_{n}=\varphi$ implies $\mathrm{w}-\lim F\left(\varphi_{n}\right)=F(\varphi)$.

Proof of Theorem 3.17. We will deduce Theorem 3.17 from Theorem B.1 for a convenient choice of the locally compact space $X$. We use the notations in Subsections 3.3 and 3.4.

Let $\chi$ be a smooth function which has a zero of order $\alpha(\xi)$ at each $\xi \in \operatorname{supp} \alpha$ and has no other zeros. This means $\chi=c_{\omega} \chi_{\omega}+o\left(\chi_{\omega}\right)$ if $\omega \in \tilde{\alpha}$ with $c_{\omega}$ non zero numbers and $\chi(x) \neq 0$ outside $\operatorname{supp} \alpha$.

Let $2 \varepsilon$ be the minimal distance between two points of $\operatorname{supp} \alpha$ and let $\theta_{0}$ be a smooth function with $\theta_{0}(x)=1$ if $|x|<\varepsilon / 3$ and $\theta_{0}(x)=0$ if $|x|>\varepsilon / 2$. Then let $\theta_{1}$ be a smooth function equal to 1 on a neighborhood of $\infty$ and equal to 0 at points at distance $<\varepsilon$ from supp $\alpha \cap \mathbb{R}$. Finally, if $\omega=(\xi, s) \in \tilde{\alpha}$ and $\xi \in \mathbb{R}$ then we set $\theta_{\omega}(x)=\theta_{0}(x-\xi)$, and if $\xi=\infty$ we set $\theta_{\omega}=\theta_{1}$. Thus the functions in the family $\left\{\theta_{\omega}\right\}_{\omega \in \tilde{\alpha}}$ have disjoint supports and each of them is equal to one on a neighborhood of a unique point from $\operatorname{supp} \alpha$.

Recall that for $\varphi \in \Lambda^{\alpha}$ we have $\delta_{\omega}(\varphi)=\delta_{\omega}\left(\varphi^{\circ}\right)$ if $\omega \prec \alpha$ and so $T_{\omega} \varphi=T_{\omega} \varphi^{\circ}$ if $\alpha \preceq \alpha$. We associate to such a $\varphi$ a function $\tilde{\varphi} \in B(\widehat{\mathbb{R}})$ defined by

$$
\tilde{\varphi}=\chi^{-1}\left(\varphi^{\circ}-\sum_{\omega \in \tilde{\alpha}} \theta_{\omega} T_{\omega} \varphi\right)
$$

outside $\operatorname{supp} \alpha$, while at points $\xi \in \operatorname{supp} \alpha$ we set

$$
\tilde{\varphi}(\xi)=c_{\omega}^{-1} \delta_{\omega}(\varphi) \quad \text { with } \omega=(\xi, \alpha(\xi)) .
$$

The definition of $\tilde{\varphi}$ on the support of $\alpha$ is such that $\tilde{\varphi} \in C(\widehat{\mathbb{R}})$ if $\varphi \in C^{\alpha}(\widehat{\mathbb{R}}) \subset \Lambda^{\alpha}$. Observe that

$$
\begin{aligned}
\sum_{\omega \in \tilde{\alpha}} \theta_{\omega} T_{\omega} \varphi & =\sum_{\mu<\omega \in \tilde{\alpha}} \theta_{\omega} \delta_{\mu}(\varphi) \chi_{\mu} \\
& =\sum_{\mu<\omega \in \alpha} \theta_{\mu} \delta_{\mu}(\varphi) \chi_{\mu} \\
& =\sum_{\omega<\alpha} \theta_{\omega} \delta_{\omega}(\varphi) \chi_{\omega},
\end{aligned}
$$

because for $\mu<\omega$ we have $\theta_{\mu}=\theta_{\omega}$. Thus we have

$$
\varphi^{\circ}=\chi \tilde{\varphi}+\sum_{\omega \in \tilde{\alpha}} \theta_{\omega} T_{\omega} \varphi=\chi \tilde{\varphi}+\sum_{\omega \prec \alpha} \theta_{\omega} \delta_{\omega}(\varphi) \chi_{\omega}
$$


Now let us denote

$$
\hat{\alpha}=\{\omega \mid \omega \prec \alpha\}
$$

and let us consider the map

$$
A: \Lambda^{\alpha}=L^{\alpha}(\widehat{\mathbb{R}}) \oplus \mathbb{C}^{\tilde{\alpha}} \longrightarrow B(\widehat{\mathbb{R}}) \oplus \mathbb{C}^{\hat{\alpha}}
$$

defined by

$$
A \varphi=\left(\tilde{\varphi},\left(\delta_{\omega}(\varphi)\right)_{\omega \prec \alpha}\right) .
$$

Then clearly $A$ is linear continuous and injective and we have

$$
A C^{\alpha}(\widehat{\mathbb{R}}) \subset C(\widehat{\mathbb{R}}) \oplus \mathbb{C}^{\hat{\alpha}} .
$$

On the other hand, from (B.1) it follows that $A$ is bijective with continuous inverse given by

$$
A^{-1}\left(\psi,\left(a_{\omega}\right)_{\omega \prec \alpha}\right)=\left(\chi \psi+\sum_{\omega \prec \alpha} \theta_{\omega} a_{\omega} \chi_{\omega},\left(c_{\omega} \psi(\omega)\right)_{\omega \in \tilde{\alpha}}\right),
$$

where we used the notation

$$
\psi(\omega)=\psi(\xi) \quad \text { for } \omega=(\xi, \alpha(\xi)) \in \tilde{\alpha} .
$$

It is also easy to check that $A^{-1}$ sends $C(\widehat{\mathbb{R}}) \oplus \mathbb{C}^{\hat{\alpha}}$ into $C^{\alpha}(\widehat{\mathbb{R}})$, hence

$$
A: C^{\alpha}(\widehat{\mathbb{R}}) \longrightarrow C(\widehat{\mathbb{R}}) \oplus \mathbb{C}^{\hat{\alpha}}
$$

is an isomorphism.

Summarizing we have

$$
A: C^{\alpha}(\widehat{\mathbb{R}}) \sim C(\widehat{\mathbb{R}}) \oplus \mathbb{C}^{\hat{\alpha}},
$$

and

$$
A: \Lambda^{\alpha} \sim B(\widehat{\mathbb{R}}) \oplus \mathbb{C}^{\hat{\alpha}} .
$$

Let $\widehat{\mathbb{R}} \sqcup \hat{\alpha}$ be the topological disjoint union of $\widehat{\mathbb{R}}$ with the discrete space $\hat{\alpha}$. We have obvious identifications

$$
C(\widehat{\mathbb{R}}) \oplus \mathbb{C}^{\hat{\alpha}} \sim C(\widehat{\mathbb{R}} \sqcup \hat{\alpha}) \quad \text { and } \quad B(\widehat{\mathbb{R}}) \oplus \mathbb{C}^{\hat{\alpha}} \sim B(\widehat{\mathbb{R}} \sqcup \hat{\alpha}),
$$

which in particular induce the natural notion of b-convergence for sequences on the space $B(\widehat{\mathbb{R}}) \oplus \mathbb{C}^{\hat{\alpha}}$. Then it is clear that $A$ and $A^{-1}$ are continuous for the bconvergence. It suffices now to apply Theorem B.1 to $X=\widehat{\mathbb{R}} \cup \hat{\alpha}$, using also Theorem 3.10. 


\section{References}

[1] W. Amrein, A. Boutet de Monvel, and W. Georgescu, $C_{0}$-groups, commutator methods and spectral theory of $N$-body Hamiltonians. Progress in Mathematics, 135. Birkhäuser Verlag, Basel, 1996. MR 1388037 Zbl 0962.47500

[2] A. Bachelot, Superradiance and scattering of the charged Klein-Gordon field by a step-like electrostatic potential. J. Math. Pures Appl. (9) 83 (2004), no. 10, 1179-1239. MR 2092306 Zbl 1063.81095

[3] J. Bognár, Indefinite inner product spaces. Ergebnisse der Mathematik und ihrer Grenzgebiete, 78. Springer, Berlin etc., 1974. MR 0467261 Zbl 0286.46028

[4] J. Derezinski and C. Gérard, Scattering theory of classical and quantum $N$-particle systems Texts and Monographs in Physics. Springer, Berlin, 1997. MR 1459161 Zbl 0899.47007

[5] S. Dyatlov, Quasi-normal modes and exponential energy decay for the Kerr-de Sitter black hole. Comm. Math. Phys. 306 (2011), no. 1, 119-163. MR 2819421 Zbl 1223.83029

[6] K. J. Eckardt, Scattering theory for the Klein-Gordon equation. Funct. Approx. Comment. Math. 8 (1980), 13-42. MR 0594117 Zbl 0493.47005

[7] R. Froese and P. Hislop, Spectral analysis of second-order elliptic operators on noncompact manifolds. Duke Math. J. 58 (1989), no. 1, 103-129. MR 1016416 Zbl 0687.35060

[8] V. Georgescu, and C. Gérard, On the virial theorem in quantum mechanics. Comm. Math. Phys. 208 (1999), no. 2, 275-281. MR 1729087 Zbl 0961.81009

[9] V. Georgescu, C. Gérard, and D. Häfner, Boundary values of resolvents of selfadjoint operators in Krein spaces. J. Funct. Anal. 265 (2013), no. 12, 3245-3304. MR 3110502 Zbl 06271944

[10] V. Georgescu, C. Gérard, and D. Häfner, Asymptotic completeness for superradiant Klein-Gordon equations and applications to the De Sitter Kerr metric. Preprint 2014. arXiv:1405.5304 [math.AP]

[11] C. Gérard, Scattering theory for Klein-Gordon equations with non-positive energy. Ann. Henri Poincaré 13 (2012), no. 4, 883-941. MR 2913626 Zbl 1253.81049

[12] C. Gérard, F. Hiroshima, A. Panati, A. Suzuki, Infrared problem for the Nelson model on static space-times. Comm. Math. Phys. 308 (2011), no. 2, 543-566. MR 2851152 Zbl 1269.83036

[13] D. Häfner, Sur la théorie de la diffusion pour l'équation de Klein-Gordon dans la métrique de Kerr. Dissertationes Math. (Rozprawy Mat.) 421 (2003), 102 pp. MR 2031494 Zbl 1075.35093

[14] D. Häfner, Complétude asymptotique pour l'équation des ondes dans une classe d'espaces-temps stationnaires et asymptotiquement plats. Ann. Inst. Fourier (Grenoble) 51 (2001), no. 3, 779-833. MR 1838466 Zbl 0981.35031 
[15] S. Itozaki, Scattering theory for Schrödinger equations on manifolds with asymptotically growing ends. Preprint 2011. arXiv:1112.5135 [math.AP]

[16] P. Jonas, On a class of selfadjoint operators in Kre in space and their compact perturbations. Integral Equations Operator Theory 11 (1988), no. 3, 351-384. MR 0938849 Zbl 0651.47020

[17] P. Jonas, Compact perturbations of definitizable operators II. J. Operator Theory 8 (1982), no. 1, 3-18. MR 0553864 Zbl 0565.47021

[18] P. Jonas, On the spectral theory of operators associated with perturbed Klein-Gordon and wave type equations. J. Operator Theory 29 (1993), no. 2, 207-224. MR 1284880 Zbl 0818.35107

[19] P. Jonas, On the functional calculus and the spectral function for definitizable operators in Krein space. Beiträge Anal. 16 (1981), 121-135. MR 0663475 Zbl 0556.47019

[20] P. Jonas and H. Langer, Compact perturbations of definitizable operators. J. Operator Theory 2 (1979), no. 1, 63-77. MR 0553864 Zbl 0478.47020

[21] H. Koch, and D. Tataru, Carleman estimates and absence of embedded eigenvalues. Comm. Math. Phys. 267 (2006), no. 2, 419-449. MR 2252331 Zbl 1151.35025

[22] H. Langer, Spectral functions of definitizable operators in Krein spaces. In D. Butković, H. Kraljević, and S. Kurepa (eds.), Functional analysis. Proceedings of the Conference held in Dubrovnik, November 2-14, 1981. Lecture Notes in Mathematics, 948. Springer, Berlin etc., 1982, 1-46. MR 0672791 MR 0672790 (collection) Zbl 0511.47023 Zbl 0482.00016

[23] H. Langer, B. Najman, C. Tretter, Spectral theory of the Klein-Gordon equation in Pontryagin spaces. Comm. Math. Phys. 267 (2006), no. 1, 159-180. MR 2238908 Zbl 1114.47038

[24] H. Langer, B. Najman, and C. Tretter, Spectral theory of the Klein-Gordon equation in Krein spaces. Proc. Edinb. Math. Soc. (2) 51 (2008), no. 3, 711-750. MR 2465932 Zbl 1152.81020

[25] L. E. Lundberg, Spectral and scattering theory for the Klein-Gordon equation. Comm. Math. Phys. 31 (1973), 243-257. MR 0334761 Zbl 1125.35391

[26] R. Melrose, Geometric scattering theory. Stanford Lectures. Cambridge University Press, Cambridge, 1995. MR 1350074 Zbl 0922.58085

[27] B. Najman, Scattering theory for matrix operators. II. Scattering theory for the abstract differential equation with a gyroscopic term. Glas. Mat. Ser. III 17(37) (1982), no. 2, 285-302. MR 0682476 Zbl 0513.47024

[28] M. Reed and B. Simon, Methods of modern mathematical physics. IV. Analysis of operators. Academic Press, New York etc., 1978. MR 0493421 Zbl 0401.47001

[29] S. Schechter, The Klein-Gordon equation and scattering theory. Ann. Physics 101 (1976), no. 2, 601-609. MR 0460926 Zbl 0347.35065

[30] I. L. Schiff, H. Snyder, and J. Weinberg, On the existence of stationary states of the mesotron field. Phys. Rev. 57 (1940), 315-318. 
[31] B. Simon, Kato's inequality and the comparison of semigroups. J. Funct. Anal. 32 (1979), no. 1, 97-101. MR 0533221 Zbl 0413.47037

[32] A. Soffer, The maximal velocity of a photon. Preprint 2011. arXiv:1103.3031 [math-ph]

[33] A. Vasy and J. Wunsch, Positive commutators at the bottom of the spectrum. J. Funct. Anal. 259 (2010), no. 2, 503-523. MR 2644111 Zbl 1194.35292

[34] K. Veselic̀ and J. Weidmann, Asymptotic estimates of wave functions and the existence of wave operators. J. Functional Analysis 17 (1974), 61-77. MR 0346342 Zbl 0286.47006

[35] R. A. Weder, Scattering theory for the Klein-Gordon equation. J. Funct. Anal. 27 (1978), no. 1, 100-117. MR 0477164 Zbl 0366.35030

[36] H. Woracek, Untitled manuscript.

http://asc.tuwien.ac.at/funkana/woracek/lva/2009S_2010S_krrr/

Received October 4, 2013; revised August 13, 2014

Vladimir Georgescu, Département de Mathématiques, UMR 8088,

Université de Cergy-Pontoise, 95000 Cergy-Pontoise Cedex, France

e-mail: Vladimir.Georgescu@math.cnrs.fr

Christian Gérard, Département de Mathématiques, Université de Paris XI, 91405 Orsay Cedex, France

e-mail: christian.gerard@math.u-psud.fr

Dietrich Häfner, Université de Grenoble 1, Institut Fourier, UMR 5582 CNRS,

BP 7438402 Saint-Martin d'Hères, France

e-mail: Dietrich.Hafner@ujf-grenoble.fr 